\title{
Quantized Enveloping Algebras Associated with Simple Lie Superalgebras and Their Universal $R$-matrices
}

\author{
By \\ Hiroyuki YAMANE*
}

\section{Introduction}

0.1. In this paper, we introduce a new family of quasi-triangular Hopf algebras coming from complex simple Lie superalgebras. We shall do this by constructing explicitly the associated universal $R$-matrices. An outline of our results has been reported in [21].

Let $\boldsymbol{H}$ be a (topological) Hopf algebra. Let $\mathscr{R}=\sum_{i} a_{i} \otimes b_{i} \in \boldsymbol{H} \otimes \boldsymbol{H}$ be an invertible element. Following Drinfeld [4], we say that $(\boldsymbol{H}, \Delta, \mathscr{R})$ is a quasi-triangular Hopf algebra if it satisfies the following properties:

$$
\bar{\Delta}(x)=\mathscr{R} \cdot \Delta(x) \cdot \mathscr{R}^{-1} \quad(x \in H),
$$

$(\Delta \otimes 1)(\mathscr{R})=\mathscr{R}_{13} \mathscr{R}_{23},(1 \otimes \Delta)(\mathscr{R})=\mathscr{R}_{13} \mathscr{R}_{12}$,

where $\bar{\Delta}=\tau \circ \Delta, \tau(x \otimes y)=y \otimes x$ and $\mathscr{R}_{12}=\sum_{i} a_{i} \otimes b_{i} \otimes 1, \mathscr{R}_{13}=\sum_{i} a_{i} \otimes 1$ $\otimes b_{i}, \mathscr{R}_{23}=\sum_{i} 1 \otimes a_{i} \otimes b_{i}$

It is easy to see that the element $\mathscr{R}$ satisfies:

$$
\mathscr{R}_{12} \mathscr{R}_{13} \mathscr{R}_{23}=\mathscr{R}_{23} \mathscr{R}_{13} \mathscr{R}_{12}
$$

Let $V$ be a finite dimensional vector space. An element $R$ of $\operatorname{End}(V) \otimes$

Communicated by M. Kashiwara, March 26, 1992.

1991 Mathematics Subject Classification: 17B37, 16W30, 17A70.

* Department of Mathematics, Faculty of Science, Osaka University, Toyonaka, Osaka 560, Japan. 
$\operatorname{End}(V)$ is called a (constant) R-mastrix if it satisfies the Yang-Baxter equation:

$$
R_{12} R_{13} R_{23}=R_{23} R_{13} R_{12}
$$

The importance of this notion in mathematics and physics is widely recognized; See, for example, [2] and [21]. From the fact (0.1.1), it follows that, if $\pi: \mathbb{H} \rightarrow$ $\operatorname{End}(V)$ is an algebra homomorphism, then $\pi \otimes \pi(\mathscr{R})$ is an $R$-matrix. For this reason the element $\mathscr{R}$ is called a universal $R$-matrix of $\mathbb{H}$.

0.2. Drinfeld [4] (and Jimbo [5]) introduced a family of quasi-triangular Hopf algebras $U_{h}(G)$ coming from complex simple Lie algebras $G_{\text {. The }}$ Hopf algebras $U_{h}(G)$ are called quantum groups or quantized enveloping algebras. Moreover Drinfeld [4] gave a method of constructing the universal $R$-matrix of $U_{h}(G)$, the so-called quantum double construction. Several authors gave explicit formulas for the universal $R$-matrix of $U_{h}(G)$ by using this method. See [8], [10], [18].

0.3. Let $\mathscr{G}$ be a complex simple Lie superalgebras of type $A-G$, and $U(\mathscr{G})$ the universal enveloping superalgebra of $\mathscr{G}$. Let $(\Phi, \mathbb{\Pi}, p)$ be a root system of $\mathscr{G}$, i.e. $\Phi, \mathbb{I}=\left\{\alpha_{1}, \cdots, \alpha_{n}\right\}$ and $p: \Pi \rightarrow\{0,1\}$ are a set of roots, a set of simple roots and a parity function respectively. In this paper, we assume that $(\Phi, \Pi, p)$ is of distinguished type (see [7]) if $\mathscr{G}$ is of type $F_{4}$ or $G_{3}$. For each such $(\Phi, \Pi, p)$, we introduce an $h$-adic topologically free $\mathbb{C}[[h]]-H o p f$ superalgebra $U_{h}(\mathscr{G})=U_{h}(\mathbb{I}, p)$ such that $U_{h}(\mathscr{G}) / h U_{h}(\mathscr{G})$ is isomorphic to $U(\mathscr{G})$ as a $\mathbb{C}$-Hopf superalgebra. The Hopf superalgebra structure of $\mathbb{U}_{h}(\mathbb{I}, p)$ seems to depend on the choice of $(\Phi, \Pi, p)$. (Note that two root systems of a simple Lie superalgebra are not necessarily isomorphic.)

0.4. Let $\mathfrak{S}=\mathfrak{S}_{0} \oplus \mathfrak{S}_{1}$ be any Hopf superalgebra. Let $\sigma: \mathfrak{S} \rightarrow \mathfrak{S}$ be an involution defined by $\sigma(x)=(-1)^{i} x$ for $x \in \mathfrak{S}_{i}$. Then $\mathfrak{T}^{\sigma}=\mathfrak{S} \rtimes\langle\sigma\rangle(\simeq \mathfrak{S} \oplus \mathfrak{S} \sigma)$ has a Hopf algebra structure (see the last paragraph of $\S 1$ ).

In this paper, we show that the Hopf algebra $U_{h}(\mathbb{I I}, p)^{\sigma}$ is quasi-triangular by constructing explicitly the associated universal $R$-matrix using the quantum double construction. As in "non-super" cases (see [8], [10], [18]), our $\mathscr{R}$ is also described by using $q$-root vectors and the $q$-exponential.

In doing these, our basic references are Lusztig's paper [11] and [12]: our $q$-root vectors are defined as natural super-versions of $q$-root vectors defined there. We also need commutation relations of $q$-root vectors similar 
to the one given in [11] and [12]. Using these results, we prove a PoincaréBirkohoff-Witt type theorem for $U_{h}(\Pi, p)$ (Theorem 10.5.1), which is almost equivalent to the topological freeness of $U_{h}(\Pi, p)$.

0.5. We define the superalgebra $U_{h}(\Pi, p)$ in a rather abstract manner in $\$ 2$. Later, we redefine it by generators and relations (see Theorem 10.5.1). A remarkable fact is that the relations are not exhausted by binary relations such as Serre relations; we also need trinomial and quadrinomial relations. Since a $C$-superalgebra $U_{h}(\Pi, p) / h U_{h}(\Pi, p)$ is isomorphic to the universal enveloping superalgebra $U(\mathscr{G})$ of $\mathscr{G}$ (see 0.2 ), we also get defining relations of $U(\mathscr{G})$ by putting $h=0$ in the relations of $U_{h}(\Pi, p)$. So we get a Serre type theorem for simple Lie superalgebras (see [6] for Serre's theorem for simple Lie algebras). This result also seems to be new.

In [9], Khoroshekin and Tolstoy "defined" their quantized Kac-Moody superalgebras by generators and relations [9; Definition 2.1]; in Note added in proof at the end of their paper, they admit that the relations given in the text of [9] are not enough. So it is not clear what they mean by "quantized Kac-Moody superalgebras". For example, the Poincaré-BirkhoffWitt type theorem does not hold, in general, for the superalgebras of Khoroshkin and Tolstoy (contrary to the remark at the end of $\S 3$ of [9]), even if the relations in Note added in proof of [9] are taken into account. See $\S 11$.

0.6. Before the Drinfeld-Jimbo quantized enveloping algebras was introduced, Perk and Shultz [15] discovered an $R$-matrix with a continuous parameter $q=e^{h}$ and a discrete parameter $\varepsilon=( \pm 1, \cdots, \pm 1) \in(\boldsymbol{Z} / 2 \boldsymbol{Z})^{N}$. For the special case $\varepsilon=(1, \cdots, 1)$, their $R$-matrix coinsides with the $R$-matrix obtained through the general procedure explained in 0.1 using the universal $R$-matrix and the fundamental representation of $U_{h}(\operatorname{gl}(N, C))$.

One of our motivation of the present work was to understand their $R$-matrix in terms of quantized enveloping algebras. In the end of $\S 10$, we show that, if $(\Pi, p)$ is of type $A_{N-1}$ and $\rho: U_{h}(\Pi, p)^{\sigma} \rightarrow M_{N}(C[[h]])$ is the fundamental representation, then $R=\rho \otimes \rho(\mathscr{R})$ is the constant $R$-matrix of Perk and Schultz, their $R$-matrix with spectral parameter being given by $R(x)=x(\rho \otimes \rho(\mathscr{R}))-x^{-1}(\rho \otimes \rho)(\mathscr{R})^{-1}$.

0.7. This paper is organized as follows. In $\S 1$, we explain the quantum double construction applied to $h$-adic topological $C[[h]]$-Hopf algebras. In 
$\S 2$, for any pair $(\Pi, p)$ of a set of simple roots $\Pi$ and a parity function $p$ of any symmetrizable Kac-Moody type Lie superalgebra, we define an $h$-adic topologically free $C[[h]]$-Hopf superalgebra $U_{h}(\Pi, p)$. We also show that, if $p(\alpha)=0$ for any $\alpha \in \Pi$, then $U_{h}(\Pi, p)$ coincides with the Drinfeld-Jimbo quantized enveloping algebra $U_{h}(G)$ defined for the Kac-Moody Lie algebra $G$ with simple roots $\Pi$.

In $\S 3-10$, for the pair $(\Pi, p)$ satisfying the assumption in 0.3 , we give the definding relations of $U_{h}(\Pi, p)$. Moreover we show $U_{h}(\Pi, p) / h U_{h}(\Pi, p)=$ $U(\mathscr{G})$ by proving the Poincare-Birkhoff-Witt type theorem for $U_{h}(\Pi, p)$.

In $\S 10$, we give an explicit formula for the universal $R$-matrix $\mathscr{R}$ of $U_{h}(\Pi, p)^{\sigma}$. The relation with the Perk-Shultz $R$-matrix is also discussed.

In $\S 11$, we remark that the trinomial and quadrinomial relations can not be dropped from our defining relations of $U_{h}(\Pi, p)$.

\section{§1. Quantum Double Construction}

1.1. Let $R=C[[h]]$ be the $C$-algebra of formal power series. We explain briefly elementary facts concerning the $h$-adic topological $R$-modules. For details, see [13].

Let $V$ be an $R$-module. Let $v_{V}=v: V \rightarrow Z_{+} \cup\{+\infty\}$ be the $h$-valuation defined as follows; if $v \in h^{i} V \backslash h^{i+1} V$, put $v(v)=i$, and, if $v \in \bigcap_{i \in \mathbb{Z}_{+}} h^{i} V$, put $v(v)=+\infty$. We can regard $V$ as a topological space such that a fundamental system of neighborhoods of $v \in V$ is given by $v+h^{i} V\left(i \in \mathbb{Z}_{+}\right)$. This topology is called the $h$-adic topology. For $v, w \in V$, we put

$$
d_{v}(v, w)=2^{-v(v-w)}
$$

Then $d_{v}($,$) is a quasi-metric for the topological space V$. For a subset $O$ of $V$, the symbol $\bar{O}$ denotes the closure of $O$. Note that any $R$-module homomorphism is continuous with respect to the $h$-adic topology.

If any Cauchy sequence (with respect to $d_{v}($,$) ) has a limit, then V$ is called complete. If $\overline{\{0\}}=\{0\}$, then $V$ is called separated. If $V$ is separated, then $d_{v}($,$) is a metric on V$. Note that, for a submodule $W$, the quotient topology of $V / W$ coincides with the $h$-adic topology of it. If $V$ is complete, then $V / W$ is also complete. If $V$ is separated and $W$ is closed, then $V / W$ is separated. 
It is well known that, for any $h$-adic topological $R$-module $V$, there exists a pair $(\hat{V}, i)$ of an $h$-adic topological $R$-module $\hat{V}$ and an $R$-homomorphism $i: V \rightarrow \hat{V}$ satisfying: For any $h$-adic topological complete separated $R$-module $W$, and, for any $R$-homomorphism $\varphi: V \rightarrow W$, there exists uniquely an $R$-homomorphism $\hat{\varphi}: \hat{V} \rightarrow W$ such that $\varphi \circ i=\hat{\varphi}$. We note that $\hat{V}$ is complete and that $i(V)$ is dense in $\hat{V}$. It is also well known that, if $V$ is separated, then $i$ is injective and the induced topology of $V(\subset \hat{V})$ coincides with the $h$-adic topology of $V$.

If a complete separated $h$-adic $R$-module $V$ has a submodule $W$ such that $W$ is a free $R$-module and $V$ is the completion of $W$, then we say that $V$ is topologically free. A basis of $W$ is called a topological basis of $V$.

Example 1.1.1. Let $V_{0}$ be a $C$-vector space and $V=R \otimes V_{0}$. Let $\hat{V}$ be the completion of $V$. Then we have the following natural identifications:

$$
\begin{aligned}
& \hat{V}=\left\{\sum_{i=0}^{\infty} h^{i} a_{i} \mid a_{i} \in V_{0}\right\}, \\
& V=\left\{\sum_{i=0}^{\infty} h^{i} a_{i} \mid a_{i} \in V_{0} \operatorname{dim}\left(\sum_{i=0}^{\infty} \boldsymbol{C} a_{i}\right)<\infty\right\}
\end{aligned}
$$

where $\sum_{i=0}^{\infty} h^{i} a_{i}$ is a formal infinite sum.

Definition 1.1.2. We say that an $R$-module $V$ has a handy basis $\left\{v_{i}\right\}_{i \in I}$ if (i) $V$ is a topologically free $R$-module with a topological basis $\left\{v_{i}\right\}_{i \in I}$, (ii) $I$ is a partially ordered set and (iii) there is an order homomorphism $p$ : $I \rightarrow \boldsymbol{Z}_{+}$such that, for each $n \in \boldsymbol{Z}_{+}, p^{-1}(n)$ is a finite set.

Example 1.1.3 Retaining the notation in Definition 1.1.2. We have natural identifications:

$$
\begin{aligned}
& \hat{V}=\left\{\sum_{i=0}^{\infty} \alpha_{i} v_{i} \mid \alpha_{i} \in R, \lim _{i \rightarrow \infty} v\left(\alpha_{i}\right)=+\infty\right\}, \\
& V=\left\{\sum_{i=0}^{\infty} \alpha_{i} v_{i} \mid \alpha_{i} \in R, \alpha_{i} \neq 0 \text { for finitely many } i, s\right\}
\end{aligned}
$$


where $\sum_{i=0}^{\infty} \alpha_{i} v_{i}$ is a formal infinite sum.

For $R$-module $V$ and $W$, we denote the completion of $V \otimes W$ by $V \hat{\otimes} W$. If $V$ and $W$ have handy bases $\left\{v_{i}\right\}_{i \in I}$ and $\left\{w_{j}\right\}_{j \in J}$ respectively, then $\left\{v_{i} \otimes w_{j}\right\}_{(i, j) \in I \times J}$ is also a handy basis of $V \otimes W$. In particular, $V \otimes W$ and $V \hat{\otimes} W$ are separated.

1.2 Let $A=(A, m, \eta, \Delta, S, \varepsilon)$ be an $h$-adic topological $R$-Hopf algebra. Namely, the $h$-adic topological $R$-module $A$ has a topological $R$-Hopf algebra structure with the product $m: A \otimes A \rightarrow A$, the unit $\eta: R \rightarrow A$, the coproduct $\Delta: A \rightarrow A \otimes A$, the antipode $S: A \rightarrow A$ and the counit $\varepsilon: A \rightarrow R$. Here the definition of the $h$-adic topological Hopf algebras is given by replacing $A \otimes A, A \otimes A \otimes A$ in the definition of the Hopf algebras by their completions $A \hat{\otimes} A, A \hat{\otimes} A \hat{\otimes} A$. For the definition of the ordinary Hopf algebras, see [1].

Define $\tau: A \hat{\otimes} A \rightarrow A \hat{\otimes} \mathrm{A}$ by $\tau(a \otimes b)=b \otimes a$. It is well known that $A^{o p}=\left(A, m, \eta, \tau \circ \Delta, S^{-1}, \varepsilon\right)$ is also an $h$-adic topological $R$-Hopf algebra. We call $A^{o p}$ the opposite Hopf algebra of $A$.

Let $\mathscr{I}$ be an ideal of the $R$-algebra $A$. We say that $\mathscr{I}$ is a bi-ideal if $\mathscr{I}$ satisfies: $\Delta(\mathscr{I}) \subseteq \mathscr{I} \otimes A+A \otimes \mathscr{I}$ and $\varepsilon(\mathscr{I})=0$. Moreover, if $\mathscr{I}$ satisfies $S(\mathscr{I})=\mathscr{I}$, we say that $\mathscr{I}$ is a Hopf ideal.

1.3. Let $A=(A, m, \eta, \Delta, S, \varepsilon)$ be an $h$-adic topological $R$-Hopf algebra. In this subsection, we assume that $A$ has a handy basis $\left\{a_{i}\right\}_{i \in I} . \quad$ Let $A^{*}=\operatorname{Hom}_{R}(A, R)$ be the dual space of the $R$-module $A$. Define $a_{i}^{*} \in A^{*}(i \in I)$ by $a_{i}^{*}\left(a_{j}\right)$ $=\delta_{i j}$. Then we have a natural identification:

$$
A^{*}=\left\{\sum_{i \in I} \alpha_{i} a_{i}^{*} \mid \alpha_{i} \in R\right\}
$$

where $\sum_{i=0}^{\infty} \alpha_{i} a_{i}^{*}$ is a formal infinite sum. Then $A^{*}$ is a torsion free complete separated $R$-module.

The $R$-module $A^{*}$ has a two-sided $A$-module structure defined by:

$$
a . f . b(c)=f(b c a) \quad\left(f \in A^{*}, a, b, c \in A\right) .
$$


Put

$$
A^{\circ}=\left\{f \in A^{*} \mid A . f . A \text { is a finitely generated free } R \text {-module }\right\} \text {. }
$$

Similarly to the case of ordinary Hopf algebras (see [1]), it can be shown easily that

$$
\begin{aligned}
A^{\circ} & =\left\{f \in A^{*} \mid A . f \quad \text { is a finitely generated free } R \text {-module }\right\} \\
& =\left\{f \in A^{*} \mid f . A \quad \text { is a finitely generated free } R \text {-module }\right\},
\end{aligned}
$$

and that $A^{\circ}=\left(A^{\circ},{ }^{t} \Delta,{ }^{t} \varepsilon,{ }^{t} m,{ }^{t} S,{ }^{t} \eta\right)$ is a non-topological $R$-Hopf algebra where $t$ denotes the transpose. We call $A^{\circ}$ the dual Hopf algebra of $A$. Let $\hat{A}^{\circ}$ be the completion of $A^{\circ}$. It is obvious that $\left(\hat{A}^{\circ},{ }^{t} \Delta,{ }^{t} \varepsilon,{ }^{t} m,{ }^{t} S,{ }^{t} \eta\right)$ is an $h$-adic $R$-Hopf algebra. We note that $\hat{A}^{\circ}$ (resp. $\hat{A}^{\circ} \hat{\otimes} \hat{A}^{\circ}, \hat{A}^{\circ} \hat{\otimes} \hat{A}^{\circ} \hat{\otimes} \hat{A}^{\circ}$ ) is naturally identified with the closure $\bar{A}^{\circ}$ of $A^{\circ}$ (resp. $\overline{A^{\circ} \otimes A^{\circ}}$ of $A^{\circ} \otimes A^{\circ}$, $\overline{A^{\circ} \otimes A^{\circ} \otimes A^{\circ}}$ of $\left.A^{\circ} \otimes A^{\circ} \otimes A^{\circ}\right)$ in $A^{*}$ (resp. $\left.(A \hat{\otimes} A)^{*},(A \hat{\otimes} A \hat{\otimes} A)^{*}\right)$. Hence we shall denote $\left(\hat{A}^{\circ},{ }^{t} \Delta,{ }^{t},{ }^{t}, m,{ }^{t} S,{ }^{t} \eta\right)$ by $\bar{A}^{\circ}$.

1.4. Let $A=(A, m, \eta, \Delta, S, \varepsilon)$ be an $h$-adic $R$-Hopf algebra. For $i \geq 2$, $\Delta^{(i)}$ and $m^{(i)}$ denote $\left(\Delta^{(i-1)} \otimes i d\right) \circ \Delta$ and $m \circ\left(m^{(i-1)} \otimes i d\right)$ respectively.

Let $A=\left(A, m_{A}, \eta_{A}, \Delta_{A}, S_{A}, \varepsilon_{A}\right)$ and $B=\left(B, m_{B}, \eta_{B}, \Delta_{B}, S_{B}, \varepsilon_{B}\right)$ be $h$-adic topological $R$-Hopf algebras. Let $\langle\rangle:, A \hat{\otimes} B \rightarrow R$ be an $R$-bilinear form. We say that $\langle$,$\rangle is a Hopf pairing if \langle$,$\rangle satisfies:$

(i) $\left\langle a_{1}, a_{2}, b\right\rangle=\left\langle a_{1} \otimes a_{2}, \Delta_{B}(b)\right\rangle$,

$$
\left\langle a, b_{1} b_{2}\right\rangle=\left\langle\Delta_{A}(a), b_{1} \otimes b_{2}\right\rangle \text {, }
$$

(ii) $\left\langle a, S_{B}(b)\right\rangle=\left\langle S_{A}(a), b\right\rangle$,

(iii) $\left\langle\eta_{A}(1), b\right\rangle=\varepsilon_{B}(b),\left\langle a, \eta_{B}(1)\right\rangle=\varepsilon_{A}(a)$,

where $a, a_{i} \in A$ and $b, b_{i} \in B$.

Define an $R$-module homomorphism $\Phi: B \hat{\otimes} A \rightarrow A \hat{\otimes} B$ by

$$
\Phi(b \otimes a)=\sum_{i, j}\left\langle a_{i}^{(3)}, b_{j}^{(1)}\right\rangle\left\langle S_{A}^{-1}\left(a_{i}^{(1)}\right), b_{j}^{(3)}\right\rangle \cdot a_{i}^{(2)} \otimes b_{j}^{(2)}
$$

where $\Delta_{A}^{(2)}(a)=\sum_{i} a_{i}^{(1)} \otimes a_{i}^{(2)} \otimes a_{i}^{(3)}$ and $\Delta_{B}^{(2)}(b)=\sum_{j} b_{j}^{(1)} \otimes b_{j}^{(2)} \otimes b_{j}^{(3)}$ 
In the following proposition, we define an $h$-adic topological $R$-Hopf algebra $D\left(A, B^{o p}\right)$ which is called the quantum double of $A$ and $B$. The notion of quantum double was introduced by Drinfeld [4] (See also [18]).

Proposition 1.4.1. Let $A$ and $B$ be h-adic $R$-Hopf algebras with a (possibly degenerate) Hopf pairing $\langle\rangle:, A \hat{\otimes} B \rightarrow R$. Then there exists uniquely an $h$-adic topological $R$-Hopf algebra $D\left(A, B^{o p}\right)=\left(D\left(A, B^{o p}\right), m_{D}, \eta_{D}\right.$, $\left.\Delta_{D}, S_{D}, \varepsilon_{D}\right)$ satisfying:

(i) As an h-adic topological $R$-module, $D\left(A, B^{o p}\right)$ is isomorphic to $A \hat{\otimes} \mathrm{B}$,

(ii) The R-module maps $A \rightarrow D\left(A, B^{o p}\right)(a \rightarrow a \otimes 1), B^{o p} \rightarrow D\left(A, B^{o p}\right)(b \rightarrow$ $1 \otimes b)$ are h-adic topological $R$-Hopf algebra homomorphisms,

(iii) The multiplication $m_{D}$ is given by $m_{D}=\left(m_{A} \otimes \mathrm{m}_{B}\right) \circ\left(i d_{A} \otimes \Phi \otimes i d_{B}\right)$.

Proof. Here we prove the associativity of the multiplication $m_{D}$ of $D\left(A, B^{o p}\right)$ only.

Let $a \otimes b, c \otimes d, e \otimes f \in D\left(A, B^{o p}\right) . \quad$ Put

$$
\begin{aligned}
& \Delta_{B}^{(2)}(b)=\sum_{u} b_{u}^{(1)} \otimes b_{u}^{(2)} \otimes b_{u}^{(3)}, \\
& \Delta_{A}^{(2)}(c)=\sum_{v} c_{v}^{(1)} \otimes c_{v}^{(2)} \otimes c_{v}^{(3)} .
\end{aligned}
$$

By (iii), we have:

$$
(a \otimes b) \cdot(c \otimes d)=\sum_{u, v}\left\langle S_{A}^{-1}\left(c_{v}^{(1)}\right), b_{u}^{(3)}\right\rangle\left\langle c_{v}^{(3)}, b_{u}^{(1)}\right\rangle a c_{v}^{(2)} \otimes b_{u}^{(2)} d
$$

Put

$$
\begin{aligned}
& \Delta_{B}^{(4)}(b)=\sum_{\zeta} b_{\zeta}^{(1)} \otimes b_{\zeta}^{(2)} \otimes b_{\zeta}^{(3)} \otimes b_{\zeta}^{(4)} \otimes b_{\zeta}^{(5)} \\
& \Delta_{B}^{(2)}(d)=\sum_{w} d_{w}^{(1)} \otimes d_{w}^{(2)} \otimes d_{w}^{(3)} \\
& \Delta_{A}^{(2)}(e)=\sum_{x} e_{x}^{(1)} \otimes e_{x}^{(2)} \otimes e_{x}^{(3)} .
\end{aligned}
$$


By (iii), we have:

$$
\begin{aligned}
(a & \otimes b) \cdot(c \otimes d)) \cdot(e \otimes f) \\
= & \sum_{v, w, x, \zeta}\left\langle S_{A}^{-1}\left(c_{v}^{(1)}\right), b_{\zeta}^{(5)}\right\rangle\left\langle c_{v}^{(3)}, b_{\zeta}^{(1)}\right\rangle \\
& \left\langle S_{A}^{-1}\left(e_{x}^{(1)}\right), b_{\zeta}^{(4)} d_{w}^{(3)}\right\rangle\left\langle e_{x}^{(3)}, b_{\zeta}^{(2)} d_{w}^{(1)}\right\rangle a c_{v}^{(2)} e_{x}^{(2)} \otimes b_{\zeta}^{(3)} d_{w}^{(2)} f .
\end{aligned}
$$

Putting

$$
\Delta_{A}^{(4)}(e)=\sum_{\xi} e_{\xi}^{(1)} \otimes e_{\xi}^{(2)} \otimes e_{\xi}^{(3)} \otimes e_{\xi}^{(4)} \otimes e_{\xi}^{(5)}
$$

we have that (1.4.2) is equal to

$$
\begin{gathered}
\sum_{v, w, x, \xi, \zeta}\left\langle S_{A}^{-1}\left(c_{v}^{(1)}\right), b_{\zeta}^{(5)}\right\rangle\left\langle c_{v}^{(3)}, b_{\zeta}^{(1)}\right\rangle \cdot\left\langle S_{A}^{-1}\left(e_{\xi}^{(1)}\right), d_{w}^{(3)}\right\rangle\left\langle S_{A}^{-1}\left(e_{\xi}^{(2)}\right), b_{\zeta}^{(4)}\right\rangle \\
\left\langle e_{x}^{(4)}, b_{\zeta}^{(2)}\right\rangle\left\langle e_{x}^{(5)}, d_{w}^{(1)}\right\rangle a c_{v}^{(2)} e_{\xi}^{(3)} \otimes b_{\zeta}^{(3)} d_{w}^{(2)} f
\end{gathered}
$$

Similarly, we can show that $(a \otimes c) \cdot((b \otimes c) \cdot(d \otimes f))$ is (1.4.3). Then

$$
((a \otimes c)(b \otimes c))(d \otimes f)=(a \otimes c)((b \otimes c)(d \otimes f))
$$

1.5. Let $V$ be a complete and separated $R$-module. Here we define the notion the convergence of a multi-series in $V$.

Let $\left\{a_{i_{1} \cdots i_{u}}\right\}_{i_{1}, \cdots, i_{u}} \in Z_{+}$be a multi-sequence in $V$. If there exists an element $\alpha \in V$ such that, for any $M \in Z_{+}$, there exists $N \in Z_{+}$satisfying that $v\left(\alpha-a_{i_{1} \cdots i_{u}}\right)>M$ for all $i_{1}, \cdots, i_{u}>N$, then we say that $\left\{a_{i_{1} \cdots i_{u}}\right\}$ converge to $\alpha$ as a multi-sequene. The element $\alpha$ is denoted by $\lim _{i_{1} \cdots i_{u}} \alpha_{i_{1} \cdots i_{u}}$. The uniqueness of $\alpha$ follows from the separatedness of $V$. The following lemma is obvious.

Lemma 1.5.1. Let $V$ be a complete separated $R$-module. Let $\left\{b_{i_{1} \cdots i_{u}}\right.$ $\left.\left(i_{1}, \cdots, i_{u} \in Z_{+}\right)\right\}$be a subset of $V$. Assume that, for any $M \in Z_{+}$, there exists $N \in Z_{+}$such that, $v\left(b_{i_{1} \cdots i_{u}}\right)>M$ if $i_{1}>N$ or, $\cdots$,or $i_{u}>N$. Then there exists the limit $\beta=\lim _{i_{1} \cdots i_{u}} \sum b_{i_{1} \cdots i_{u}}$. Moreover, for any permutation $\rho$ of, $\{1,2, \cdots, u\}$, it holds that 


$$
\lim _{i_{\rho(i)}}\left(\lim _{i_{\rho(2)}} \cdots\left(\lim _{i_{\rho(u)}} \sum b_{i_{1} \cdots i_{u}}\right) \cdots\right)=\beta
$$

1.6. Let $V$ be a complete separated $h$-adic topological $R$-module with a handy basis $\left\{v_{i}\right\}_{i \in I}$. Let $K=\mathbb{C}((h))\left(=\left\{\sum_{i=n}^{\infty} a_{i} h^{i}(n \in \mathbb{Z})\right\}\right)$ be the fraction field of $R=C[[h]]$. The $h$-valuation $v_{K}: K \rightarrow \mathbb{Z}$ is defined by putting $v_{K}\left(\sum\right.$ $\left.a_{i} h^{i}\right)=\min \left\{i \mid a_{i} \neq 0\right\}$. Let $V^{K}=K \otimes V$ be the scalar extention. Then we have the following identification:

$$
V^{K}=\left\{\sum_{i \in I} \alpha_{i} v_{i} \mid \alpha_{i} \in \mathrm{K}, \lim _{i \rightarrow+\infty} v\left(\alpha_{i}\right)=+\infty\right\}
$$

The $h$-valuation $v_{V K}$ on $V^{K}$ is given by putting

$$
v_{V^{K}}\left(\sum \alpha_{i} v_{i}\right)=\min \left\{v_{K}\left(\alpha_{i}\right) \mid i \in I\right\}
$$

Let $A$ and $B$ be complete separated $h$-adic topological $R$-Hopf algebras with a non-degenerate Hopf pairing $\langle\rangle:, A \hat{\otimes} B \rightarrow R$. We assume that $A$ (resp. $B$ ) has a handy basis $\left\{a_{i}\right\}_{i \in I}$ (resp. $\left\{b_{i}\right\}_{i \in I}$ ). Moreover we assume that $\left\langle a_{i}, b_{j}\right\rangle=\delta_{i j} c_{i}$ for some $c_{i} \in R \backslash\{0\}$. Let $D=D\left(A, B^{o p}\right)$ be the quantum double.

Let $A^{K}, B^{K}, D^{K}$ be $h$-adic topological $K$-Hopf algebras which are obtained by the scalar extentions of $R$-modules $A, B, D$ respectively. Then $D^{K} \simeq A^{K} \hat{\otimes} \mathrm{B}^{K}$ as an $h$-adic topological $K$-vector spaces. Moreover we consider $A^{K}$ (resp. $\left(B^{K}\right)^{o p}$ ) as a topological $K$-Hopf subalgebra of $D^{K}$ by the embedding $A^{K} \rightarrow D^{K}(a \rightarrow a \otimes 1)$ (resp. $\left.\left(B^{K}\right)^{o p} \rightarrow D^{K}(b \rightarrow 1 \otimes b)\right)$. We denote the Hopf pairing $K \otimes\langle\rangle:, A^{K} \otimes B^{K} \rightarrow R$ simply by $\langle$,$\rangle .$

Let $\left\{e_{i}\right\}_{i \in I}$ and $\left\{e^{i}\right\}_{i \in I}$ be the subsets of $A^{K}$ and $B^{K}$ respectively such that $e_{i} \in K a_{i}, e^{i} \in K b_{i}$ and $\left\langle e_{i}, e^{j}\right\rangle=\delta_{i j}$. Let us define $m_{j}^{i_{1} \cdots i_{u}}, \mu_{j_{1} \cdots j_{v}}^{i}, \gamma_{j}^{i} \in K$ by

$$
\begin{aligned}
& m_{A}^{(u-1)}\left(e_{i_{1}} \otimes \cdots \otimes e_{i_{u}}\right)=\sum m_{j}^{i_{1} \cdots i_{u}} e_{j}, \\
& \Delta_{A^{K}}^{(v-1)}\left(e_{j}\right)=\sum \mu_{j_{1} \cdots j_{v}}^{i} e_{j_{1}} \otimes \cdots \otimes e_{j_{v}}, \\
& S_{A^{K}}^{-1}\left(e_{i}\right)=\sum \gamma_{j}^{i} e_{j} .
\end{aligned}
$$

Using Hopf pairings $\langle$, $\rangle$, we have:

$$
\begin{aligned}
& \Delta_{B}^{(u-1)}\left(e^{j}\right)=\sum m_{j}^{i_{1} \cdots i_{u}} e_{i_{1}} \otimes \cdots \otimes e_{i_{u}}, \\
& m_{B}^{(v-1)}\left(e^{j_{1}} \otimes \cdots \otimes e^{j_{v}}\right)=\sum \mu_{j_{1} \cdots j_{v}}^{i} e^{i}
\end{aligned}
$$




$$
S_{B^{K}}^{-1}\left(e^{i}\right)=\sum \gamma_{i}^{j} e^{j}
$$

From Proposition 1.4.1, we obtain the following lemma. We omit the proof.

Lemma 1.6.1. In $D^{K}$, the following equations hold.

(i) $e^{t} e_{s}=\sum \mu_{n j k}^{s} m_{t}^{k l p} \gamma_{p}^{n} e_{j} e^{l}$.

(ii) $e_{s} e^{t}=\sum \mu_{k j n}^{s} m_{t}^{p l k} \gamma_{p}^{n} e_{j}^{l}$.

1.7. In [4], Drinfeld introduced the following construction of a universal $R$-matrix, which are so-called the quantum double construction.

Proposition 1.7.1 (The quantum double construction). Retaining the notation in 1.6. Let $C$ be a complete separated h-adic topological $R$-algebras with 1 . Let $\Omega: D \rightarrow C$ be an R-algebra homomorphism. Denote the scalar extention $i_{K} \otimes \Omega$ : $D^{K} \rightarrow C^{K} \quad$ by again $\Omega$. Assume that $\mathscr{R}=\sum_{i \in I} \Omega\left(e_{i}\right) \otimes \Omega\left(e^{i}\right)$ converges in $C^{K} \hat{\otimes} C^{K} . \quad$ Then $\mathscr{R}$ satisfies:

(i) The element $\mathscr{R}$ is invertible. The inverse $\mathscr{R}^{-1}$ is given by $\mathscr{R}=$ $\sum_{i \in I} \Omega\left(S\left(e_{i}\right)\right) \otimes \Omega\left(e^{i}\right)$,

(ii) $\mathscr{R}(\Omega \otimes \Omega(\Delta(x))) \mathscr{R}^{-1}=\Omega \otimes(\tau \circ \Delta(x))$ for all $x \in D^{K}$,

(iii) $\sum_{i \in I}(\Omega \otimes \Omega \otimes \Omega)\left((\Delta \otimes i d)\left(e_{i} \otimes e^{i}\right)\right)=\mathscr{R}_{13} \mathscr{R}_{23}$, $\sum_{i \in I}(\Omega \otimes \Omega \otimes \Omega)\left((i d \otimes \Delta)\left(e_{i} \otimes e^{i}\right)\right)=\mathscr{R}_{13} \mathscr{R}_{12}$.

Proof. Note that any multi-series below satisfies the assumption in Lemma 1.5.1. In the proof, we simply write $a \otimes b$ for $(\Omega \otimes \Omega)(a \otimes b)$. For all $i \in I$, we have:

$$
\begin{aligned}
& \mathscr{R} \cdot \Delta\left(e_{i}\right)=\sum \mu_{r s}^{i} e_{t} e_{r} \otimes e^{t} e_{s} \\
= & \sum \mu_{r s}^{i} m_{x}^{t r} \mu_{n j k}^{s} m_{t}^{k l p} \gamma_{p}^{n} e_{x} \otimes e_{j} e^{l}=\sum \mu_{r n j k}^{i} m_{x}^{k l p r} \gamma_{p}^{n} e_{x} \otimes e_{j} e^{l} \\
= & \sum \mu_{j k}^{i} m_{x}^{k l} e_{x} \otimes e_{j} e^{l}=\sum \mu_{j k}^{i} e_{k} e_{l} \otimes e_{j} e^{l}=\left(\tau \circ\left(\mathrm{e}_{i}\right)\right) \cdot \mathscr{R}
\end{aligned}
$$


Similarly, we have $\mathscr{R} \cdot \Delta\left(e^{i}\right)=\tau \circ\left(e^{i}\right) \cdot \mathscr{R}$ for all $i \in I$. Since $\left\{a_{i} \cdot b_{j}=a_{i} \otimes b_{j} \in\right.$ $\left.R e_{i} \cdot e^{j}\right\}_{(i, j) \in I \times I}$ is a topological basis of $D^{k}$ we obtain (ii). The equations (i), (iii) can be proved similarly.

1.8. Here, we comment on the definition of an $h$-adic topological algebra with generators and relations.

Let $V$ be a free $R$-module with a basis $\left\{x_{l}\right\}_{l \in L}$. Let $\mathscr{F}=R\left\langle x_{l} \mid l \in \mathbb{L}\right\rangle$ be the tensor algebra $T(V)$ of $V$ and let $\hat{\mathscr{F}}$ be the completion of $\mathscr{F}$. Let $P_{\lambda}(\lambda \in \Lambda)$ be the elements of $\hat{\mathscr{F}}$. Put $\mathscr{C}=\hat{\mathscr{F}} /\left(\sum_{\lambda} \mathscr{F} \cdot P_{\lambda} \cdot \mathscr{F}\right)$. We say that the $h$-adic topological $R$-algebra $\mathscr{C}$ is $h$-adically generated by $x_{l}(l \in L)$ with the relations $\left\{P_{\lambda}(\lambda \in \Lambda)\right\}$.

1.9. Let $\mathfrak{H}=\mathfrak{H}_{0} \oplus \mathfrak{H}_{1}$ be a (topological) $R$-superalgebra. For $i \in\{0,1\}$, define $p_{i}: \mathfrak{H} \rightarrow \mathfrak{H}_{i}$ by $p_{i}\left(x_{0}+x_{1}\right)=x_{i}$ where $x_{k} \in \mathfrak{H}_{k}(k \in\{0,1\})$. Let $\langle\sigma\rangle$ be the cyclic group of order two with a generator $\sigma$. Let $R\langle\sigma\rangle$ be the group ring of $\langle\sigma\rangle$ over $R$. We define an $R$-algebra structure on an $R$-module $\mathfrak{H}^{\sigma}=\mathfrak{T} \otimes_{R}$ $R\langle\sigma\rangle$ by

$$
\left(x \otimes \sigma^{c}\right)\left(y \otimes \sigma^{d}\right)=x\left(p_{0}(y)+(-1)^{c} p_{1}(y)\right) \otimes \sigma^{c+d} .
$$

We write $x \sigma^{c}$ for $x \otimes \sigma^{c}$. Define $r_{\sigma}: \mathfrak{H} \rightarrow \mathfrak{H}^{\sigma}\left(\right.$ resp. $\left.l_{\sigma}: \mathfrak{H} \rightarrow \mathfrak{H}^{\sigma}\right)$ by $r_{\sigma}(x)=x \sigma$ (resp. $\left.l_{\sigma}(x)=\sigma x\right)(x \in \mathfrak{H})$. From the axiom of Hopf superalgebras (see [17]), we can easily show:

Proposition 1.9.1. Let $\left(\mathfrak{S}=\mathfrak{S}_{0} \oplus \mathfrak{H}_{1}, \dot{\Delta}, \dot{\varepsilon}, \dot{S}\right)$ be a (topological) $R$-Hopf superalgebra. Then the R-algebra $\mathfrak{S}^{\sigma}$ has a (topological) $R$-Hopf algebra structure $\left(\mathfrak{S}^{\sigma}, \Delta, \varepsilon, S\right)$ such that

(i) The coproduct $\Delta: \mathfrak{H}^{\sigma} \rightarrow \mathfrak{H}^{\sigma} \otimes \mathfrak{S}^{\sigma}$ is defined by $\Delta(x)=\left(\left(i d \otimes p_{0}+\right.\right.$ $\left.\left.r_{\sigma} \otimes p_{1}\right) \circ \dot{\Delta}\right)(x)(x \in \mathfrak{G})$ and $\Delta(\sigma)=\sigma \otimes \sigma$,

(ii) The counit $\varepsilon: \mathfrak{H}^{\sigma} \rightarrow R$ is defined by $\varepsilon(x)=\dot{\varepsilon}(x)(x \in \mathfrak{S})$ and $\varepsilon(\sigma)=1$,

(iii) The antipode $S: \mathfrak{S}^{\sigma} \rightarrow \mathfrak{H}^{\sigma}$ is defined by $S(x)=\left(\left(p_{0}+l_{\sigma} \circ p_{1}\right) \circ \dot{S}\right)(x)$ $(x \in \mathfrak{H})$ and $S(\sigma)=\sigma$.

Conversely, we can also show:

Proposition 1.9.2. Let $\mathfrak{H}$ be a (topological) $R$-superalgebra. Assume that $\mathfrak{H}^{\sigma}$ has a (topological) R-Hopf algebra structure $\left(\mathfrak{S}^{\sigma}, \triangle, \varepsilon, S\right)$ satisfying: 
(i) $\quad \Delta\left(\mathfrak{H}_{0}\right) \subset \mathfrak{S}_{0} \otimes \mathfrak{H}_{0}+\mathfrak{H}_{1} \sigma \otimes \mathfrak{H}_{1}$, $\Delta\left(\mathfrak{S}_{1}\right) \subset \mathfrak{H}_{1} \otimes \mathfrak{H}_{0}+\mathfrak{H}_{0} \sigma \otimes \mathfrak{H}_{1}$ and $\Delta(\sigma)=\sigma \otimes \sigma$,

(ii) $\varepsilon\left(\mathfrak{S}_{0}\right)=R, \varepsilon\left(\mathfrak{H}_{1}\right)=\{0\}$ and $\varepsilon(\sigma)=1$,

(iii) $S\left(\mathfrak{H}_{0}\right) \subset \mathfrak{S}_{0}, S\left(\mathfrak{H}_{1}\right) \subset \sigma \mathfrak{S}_{1}$ and $S(\sigma)=\sigma$.

Then there uniquely exists a (topological) $R$-Hopf superalgebra structure $(\mathfrak{H}, \dot{\Delta}, \dot{\varepsilon}, \dot{S})$ such that $\left(\mathfrak{H}^{\sigma}, \Delta, \varepsilon, S\right)$ coincides with the Hopf algebra defined in Proposition 1.9.1 for $(\mathfrak{S}, \dot{\Delta}, \dot{\varepsilon}, \dot{S})$.

\section{§2. Quantized Enveloping (Super)algebras}

Notation. In $\S 2-10$, the following notation will be used:

$(\mathscr{E}, \Pi, p):=\mathrm{a}$ triple of an $N$-dimensional $C$-vector space $\mathscr{E}$ with a non-degenerate symmetric bilinear form (, ): $\mathscr{E} \times \mathscr{E} \rightarrow C$, a linearly independent finite subset $\Pi=\left\{\alpha_{1}, \cdots, \alpha_{n}\right\}$ of $\mathscr{E}$ and a function $p: \Pi \rightarrow\{0,1\}$ (see 2.1)

$P_{+}:=\boldsymbol{Z}_{+} \alpha_{1} \oplus \boldsymbol{Z}_{+} \alpha_{2} \oplus \cdots \oplus \boldsymbol{Z}_{+} \alpha_{n}(\subset \mathscr{E})$ (see 2.3)

$\boldsymbol{D}=\operatorname{diag}\left(d_{1}, \cdots, d_{n}\right):=$ a diagonal matrix of degree $n$ whose matrix elements are half integers (see 2.1)

$\mathscr{H}:=\mathscr{E}^{*}$ (see 2.1)

$H_{\lambda}(\lambda \in \mathscr{E}):=$ the element of $\mathscr{H}$ defined by $\mu\left(H_{\lambda}\right)=(\mu, \lambda)(\mu \in \mathscr{E})$ (see 2.1)

$R:=C[[h]]$, the ring of formal power series in an indeterminate $h$

$q:=e^{h} \in R$ (see 2.9)

$\tilde{N}_{+}:=$a free $R$-algebra with generators $\left\{E_{i} \mid 1 \leq i \leq n\right\}$ (see 2.1)

$\tilde{N}_{-}:=$a free $R$-algebra with generators $\left\{F_{i} \mid 1 \leq i \leq n\right\}$ (see 2.1)

$\Im\left[\mathscr{H}^{R}\right]:=$ a symmetric $R$-algebra generated by $\mathscr{H}^{R}=\mathscr{H} \otimes R$ (see 2.1)

$R\langle\sigma\rangle:=$ a group ring over $R$ of a cyclic group $\langle\sigma\rangle$ of order two (see 2.1)

$\tilde{U}_{h}^{\sigma}=\tilde{U}_{h}^{\sigma}((\mathscr{E}, \Pi, p), \boldsymbol{D}):=$ an $h$-adic $R$-Hopf algebra, which is, as an $R$-module, isomorphic to $\tilde{N}_{+} \hat{\otimes} \subseteq\left[\mathscr{H}^{R}\right] \hat{\otimes} R\langle\sigma\rangle \hat{\otimes} \tilde{N}_{-}$(see Lemma 2.1.4)

$R^{\prime}:=C[[\sqrt{h}]]($ see 2.2$)$

$\tilde{N}_{+}^{\prime}:=\tilde{N}_{+} \otimes R^{\prime}($ see 2.2$)$

$\Im\left[\mathscr{H}^{R^{\prime}}\right]:=\Im\left[\mathscr{H}^{R}\right] \otimes R^{\prime}($ see 2.2)

$R^{\prime}\langle\sigma\rangle:=R\langle\sigma\rangle \otimes R^{\prime}$ (see 2.2)

$\tilde{U}_{\sqrt{h}}^{\prime} b_{+}^{\sigma}=\tilde{U}_{\sqrt{h}}^{\prime} b_{+}^{\sigma}(\mathscr{E}, \Pi, p):=a \sqrt{h}$-adic $R^{\prime}$-Hopf algebra, which is as an $R^{\prime}$-module, isomorphic to $\tilde{N}_{+}^{\prime} \otimes \subseteq\left[\mathscr{H}^{R^{\prime}}\right] \otimes R^{\prime}\langle\sigma\rangle$; We put $E_{i}^{\prime}=E_{i} \otimes 1 \otimes 1$, 
$\sigma^{\prime}=1 \otimes 1 \otimes \sigma, H^{\prime}=1 \otimes H \otimes 1(H \in \mathscr{H})$; usually we identify $E_{i}^{\prime}$ and $\sigma^{\prime}$ with $E_{i}$ and $\sigma$ (but not $H^{\prime}$ with $H$ ) (see 2.2)

$E_{i}^{\circ}, H_{\lambda}^{\prime \circ}, \sigma^{\circ}:=$ elements of $\overline{\left(\widetilde{U}_{\sqrt{h}}^{\prime} b_{+}^{\sigma}\right)^{\circ}}$ (see 2.3.1-3))

$\langle\rangle:, \widetilde{U}_{\sqrt{h}}^{\prime} b_{+}^{\sigma} \times{\widetilde{U^{h}}}_{\sqrt{h}}^{\prime} b_{+}^{\sigma} \rightarrow R^{\prime}:=$ a Hopf paring (see 2.4)

$\tilde{D}^{\prime}:=D\left(\widetilde{U}_{\sqrt{h}}^{\prime} b_{+}^{\sigma},\left(\widetilde{U}_{\sqrt{h}}^{\prime} b_{+}^{\sigma}\right)^{o p}\right)$ the quantum double defined with respect to $\langle\rangle,($ see 2.5$)$

Since, as $R^{\prime}$-modules, $\mathfrak{D}^{\prime} \simeq \widetilde{U}_{\sqrt{h}}^{\prime} b_{+}^{\sigma} \otimes \widetilde{U}_{\sqrt{h}}^{\prime} b_{+}^{\sigma}$, we write $X \in \widetilde{D}^{\prime}$ for $X \otimes 1$ and $X^{\circ} \in \widetilde{D}^{\prime}$ for $1 \otimes X$ where $X \in \widetilde{U}_{\sqrt{h}}^{\prime} b_{+}^{\sigma}$ (see 2.5)

$I_{b_{+}}^{\prime}:=\operatorname{Ker}\langle$,$\rangle (see 2.6)$

$U_{\sqrt{h}}^{\prime} b_{+}^{\sigma}:=\widetilde{U}_{\sqrt{h}}^{\prime} b_{+}^{\sigma} / I_{b_{+}}^{\prime}($ see 2.7$)$

$I_{+}^{\prime}:=\operatorname{Ker}\langle,\rangle_{\mid \tilde{N}_{+}^{\prime} \times \tilde{N}_{+}^{\prime}}($ see 2.6)

$N_{+}^{\prime}:=\tilde{N}_{+}^{\prime} / I_{+}^{\prime}($ see 2.7$)$

$\mathfrak{D}^{\prime}:=D\left(U_{\sqrt{h}}^{\prime} b_{+}^{\sigma},\left(U_{\sqrt{h}}^{\prime} b_{+}^{\sigma}\right)^{o p}\right)$ the quantum double defined with respect to $\langle$,$\rangle (see 2.8)$

Since, as $R^{\prime}$-modules, $\mathfrak{D}^{\prime} \simeq U_{\sqrt{h}}^{\prime} b_{+}^{\sigma} \otimes U_{\sqrt{h}}^{\prime} b_{+}^{\sigma}$, we write $X \in \mathfrak{D}^{\prime}$ for $X \otimes 1$ and $X^{\circ} \in \mathfrak{D}^{\prime}$ for $1 \otimes X$ where $X \in U_{\sqrt{h}}^{\prime} b_{+}^{\sigma}$ (see 2.5)

$N_{+}:=$a unital $R$-subalgebra of $N_{+}^{\prime}$ such that $N_{+}^{\prime}=N_{+} \oplus \sqrt{h} N_{+}$(see Lemma 2.9.1)

$$
I_{+}:=I_{+}^{\prime} \cap \tilde{N}_{+} ; I_{+}^{\prime}=I_{+} \oplus \sqrt{h} I_{+}, N_{+}=\tilde{N}_{+} / I_{+} \text {(see Lemma 2.9.1) }
$$

$U_{h}^{\sigma}=U_{h}^{\sigma}((\mathscr{E}, \Pi, p), D):=$ a topologically free $R$-Hopf algebra (see Theorem 2.9.4); if $\Pi$ is a set of a simple roots of a Kac-Moody Lie algebra $G$ (resp. a simple Lie superalgebra $\mathscr{G}$ in 3.1) and $p\left(\alpha_{i}\right)=0$ for all $\alpha_{i} \in \Pi$, then, as a $C$-Hopf algebra, $U_{h}^{\sigma} / h U_{h}^{\sigma} \simeq U(G)^{\sigma}\left(\right.$ resp. $\left.U_{h}^{\sigma} / h U_{h}^{\sigma} \simeq U(\mathscr{G})^{\sigma}\right)$ (see Theorem 2.10 .1 (resp. Theorem 10.5.1))

$\mathscr{I}_{+}:=$an ideal of $\tilde{N}_{+}$generated by Serre relations and additional relations (see Definition 4.2.1)

$\mathscr{N}_{+}:=\tilde{N}_{+} / \mathscr{I}_{+}($see 4.3$)$

$\mathscr{I}_{b_{+}}^{\prime}:=$ an ideal of $\widetilde{U}_{\sqrt{h}}^{\prime} b_{+}^{\sigma}$ generated by elements of $\mathscr{I}_{+}$(see 4.3)

$\mathscr{U}_{\sqrt{h}}^{\prime} b_{+}^{\sigma}:=\widetilde{U}_{\sqrt{h}}^{\prime} b_{+}^{\sigma} / \mathscr{I}_{b_{+}}^{\prime}($ see 4.3$)$

In fact, it will be shown that $\mathscr{I}_{+}=I_{+}, \mathscr{I}_{b_{+}}^{\prime}=I_{b_{+}}^{\prime}, \mathscr{U}_{\sqrt{h}}^{\prime} b_{+}^{\sigma}=U_{\sqrt{h}}^{\prime} b_{+}^{\sigma}$ (see Proposition 10.4.1)

$\mathscr{N}_{+, v}:=$ a weight space of $\mathscr{N}_{+}$of a weight $v \in P_{+}$(see 4.4)

2.1. In $\S 2$, we construct quantized enveloping algebras associated with 
generalized symmetrizable Cartan matrices of Kac-Moody type Lie superalgebras.

Let $\mathscr{E}$ be an $N$-dimensional complex linear space with a non-degenerate symmetric bilinear form $():, \mathscr{E} \times \mathscr{E} \rightarrow C$. Let $\Pi=\left\{\alpha_{1}, \cdots, \alpha_{n}\right\}$ be a finite linearly independent subset of $\mathscr{E}$. We call a function $p: \Pi \rightarrow\{0,1\}$ the parity function. We call $(\mathscr{E}, \Pi, p)$ a triple system. Let $d_{i} \in \frac{1}{2} Z \backslash\{0\}(1 \leq i \leq n)$. Define the diagonal matrix $\boldsymbol{D}$ by $\operatorname{diag}\left(d_{1}, \cdots, d_{n}\right)$. Put $\mathscr{H}=\mathscr{E}^{*}$. For $\lambda \in \mathscr{E}$, let us define $H_{\lambda} \in \mathscr{H}$ by $\mu\left(H_{\lambda}\right)=(\mu, \lambda)$ for all $\mu \in \mathscr{E}$.

Let $\tilde{U}_{h}^{\sigma}=\tilde{U}_{h}^{\sigma}((\mathscr{E}, \Pi, p), D)$ be an $h$-adic topological $R$-algebra $h$-adically defined with generators $E_{i}, F_{i}(1 \leq i \leq n), H \in \mathscr{H}, \sigma$ and relations: (Here $[X, Y]$ denotes $X Y-Y X$.)

$$
\begin{aligned}
& \sigma^{2}=1, \sigma H \sigma=H(H \in \mathscr{H}), \sigma E_{i} \sigma=(-1)^{p\left(\alpha_{i}\right)} E_{i}, \\
& \sigma F_{i} \sigma=(-1)^{p\left(\alpha_{i}\right)} F_{i} \\
& {\left[H_{1}, H_{2}\right]=0\left(H_{1}, H_{2} \in \mathscr{H}\right),} \\
& {\left[H, E_{i}\right]=\alpha_{i}(H) E_{i},\left[H, F_{i}\right]=-\alpha_{i}(H) F_{i}(H \in \mathscr{H}),} \\
& E_{i} F_{j}=-(-1)^{p\left(\alpha_{i}\right) p\left(\alpha_{j}\right)} F_{j} E_{i}=\delta_{i j} \frac{\operatorname{sh}\left(h H_{\alpha_{i}}\right)}{\operatorname{sh}\left(h d_{i}\right)}
\end{aligned}
$$

Similarly to [19], we have:

Lemma 2.1.4. (The triangular decomposition of $\left.\tilde{U}_{h}^{\sigma}\right)$ Let $\tilde{N}_{+},\left(\right.$resp. $U\left(\mathscr{H}^{R}\right)$, $R\langle\sigma\rangle$ or $\left.\tilde{N}_{-}\right)$be the unital R-subalgebra of $\tilde{U}_{h}^{\sigma}$ algebraically generated by the elements $\left\{E_{1}, \cdots, E_{n}\right\}$ (resp. $\left\{H_{1}, \cdots, H_{N}\right\},\{\sigma\}$ or $\left\{F_{1}, \cdots, F_{n}\right\}$ ). Then $\tilde{N}_{+}$(resp. $\left.\tilde{N}_{-}\right)$is isomorphic to the free algebra $R\left\langle E_{1}, \cdots, E_{n}\right\rangle$ (resp. $\left.R\left\langle F_{1}, \cdots, F_{n}\right\rangle\right)$. The algebra $U\left(\mathscr{H}^{R}\right)$ is isomorphic to the symmetric algebra $\subseteq\left[\mathscr{H}^{R}\right]$ of the R-module $\mathscr{H}^{R}=R \otimes \mathscr{H}$. The R-module $R\langle\sigma\rangle$ is isomorphic to $R \sigma \oplus R$. Moreover we have an isomorphism of $h$-adic topological $R$-modules: $(c=0,1)$.

$$
\tilde{N}_{+} \hat{\otimes} \subseteq[\mathscr{H}] \hat{\otimes} R\langle\sigma\rangle \hat{\otimes} \tilde{N}_{-} \rightarrow \tilde{U}_{h}^{\sigma}\left(X \otimes Z \otimes \sigma^{c} \otimes Y \rightarrow X \cdot Z \cdot \sigma^{c} \cdot Y\right)
$$

Proof. Let $R\left\langle x_{1}, \cdots, x_{n}\right\rangle$ and $R\left\langle y_{1}, \cdots, y_{n}\right\rangle$ be the tensor algebras of the free $R$-modules with bases $x_{1}, \cdots, x_{n}$ and $y_{1}, \cdots, y_{n}$ respectively. Let $z_{1}, \cdots, z_{N}$ be a basis of $\mathscr{H}$. Put $V=\tilde{N}_{+} \hat{\otimes} \subseteq\left[\mathscr{H}^{R}\right] \hat{\otimes} \mathrm{R}\langle\sigma\rangle \hat{\otimes} \tilde{N}_{-}$. Note that the topological basis $\left\{x_{i_{1}} \cdots x_{i_{u}} \otimes z_{1}^{a_{1}} \cdots z_{N}^{a_{N}} \otimes \sigma^{c} \otimes y_{j_{1}} \cdots y_{j_{v}}\right\}$ is a handy basis with $I=\left\{\left(i_{1}, \cdots, i_{u}, a_{1}, \cdots, a_{N}, c, j_{1}, \cdots, j_{v}\right)\right\}$ and $p\left(\left(i_{1}, \cdots, i_{u}, a_{1}, \cdots, a_{N}, c, j_{1}, \cdots, j_{v}\right)\right)=i_{1}+\cdots$ 
$+i_{u}+a_{1}+\cdots+a_{N}+c+j_{1}+\cdots+j_{v}$. We can define a $\tilde{U}_{h}^{\sigma}$-module structure on $V$ by the following formulas: (Here $p(i)$ denotes $p\left(\alpha_{i}\right)$.)

$$
\begin{aligned}
& F_{i} \cdot x_{i_{1}} \cdots x_{i_{u}} \otimes z_{1}^{a_{1}} \cdots z_{N}^{a_{N}} \otimes \sigma^{c} \otimes y_{j_{1}} \cdots y_{j_{v}} \\
& =-\sum_{s=1}^{u} \delta_{i_{s}, i}(-1)^{p(i)\left\{p\left(i_{1}\right)+\cdots+p\left(i_{s}-1\right)\right\}} \\
& x_{i_{1}} \cdots \hat{x}_{i_{s}} \cdots x_{i_{u}} \otimes \frac{\operatorname{sh}\left(h\left(H_{\alpha_{i}}-\left(\alpha_{i_{s}+1}+\cdots+\alpha_{i_{u}}\left(H_{\alpha_{i}}\right)\right)\right)\right.}{\operatorname{sh}\left(h d_{i}\right)} z_{1}^{a_{1}} \cdots z_{N}^{a_{N}} \otimes \sigma^{c} \otimes y_{j_{1}} \cdots y_{j_{v}} \\
& +(-1)^{p(i)\left\{p\left(i_{1}\right)+\cdots+p\left(i_{u}\right)+c\right\}} \\
& x_{i_{1}} \cdots x_{i_{u}} \otimes\left(z_{1}+\alpha_{i}\left(z_{1}\right)\right)^{a_{1}} \cdots\left(z_{N}+\alpha_{i}\left(z_{N}\right)\right)^{a_{N}} \otimes \sigma^{c} \otimes y_{i} y_{j_{1}} \cdots y_{j_{v}}(1 \leq i \leq n), \\
& H \cdot x_{i_{1}} \cdots x_{i_{u}} \otimes z_{1}^{a_{1}} \cdots z_{N}^{a_{N}} \otimes \sigma^{c} \otimes y_{j_{1}} \cdots y_{j_{v}} \\
& =x_{i_{1}} \cdots x_{i_{u}} \otimes\left(H+\left(\alpha_{i_{1}}+\cdots+\alpha_{i_{u}}\right)(H)\right) z_{1}^{a_{1}} \cdots z_{N}^{a_{N}} \otimes \sigma^{c} \otimes y_{j_{1}} \cdots y_{j_{v}}(H \in \mathscr{H}) \text {, } \\
& \sigma \cdot x_{i_{1}} \cdots x_{i_{u}} \otimes z_{1}^{a_{1}} \cdots z_{N}^{a_{N}} \otimes \sigma^{c} \otimes y_{j_{1}} \cdots y_{j_{v}} \\
& =(-1)^{p\left(i_{1}\right)+\cdots+p\left(i_{u}\right)} x_{i_{1}} \cdots x_{i_{u}} \otimes z_{1}^{a_{1}} \cdots z_{N}^{a_{N}} \otimes \sigma^{c+1} \otimes y_{j_{1}} \cdots y_{j_{v}} \text {, } \\
& E_{i} \cdot x_{i_{1}} \cdots x_{i_{u}} \otimes z_{1}^{a_{1}} \cdots z_{N}^{a_{N}} \otimes \sigma^{c} \otimes y_{j_{1}} \cdots y_{j_{v}} \\
& =x_{i} x_{i_{1}} \cdots x_{i_{u}} \otimes z_{1}^{a_{1}} \cdots z_{N}^{a_{N}} \otimes \sigma^{c} \otimes y_{j_{1}} \cdots y_{j_{v}}(1 \leq i \leq n) \text {. }
\end{aligned}
$$

On the other hand, by using (2.1.1)-(2.1.3), we can show that $\tilde{U}_{h}^{\sigma}$ is generated by the elements

$$
E_{i_{1}} \cdots E_{i_{u}} \cdot z_{1}^{a_{1}} \cdots z_{N}^{a_{N}} \cdot \sigma^{c} \cdot F_{j_{1}} \cdots F_{j_{v}}\left(a_{1}, \cdots, a_{n} \in Z_{+}, c \in\{0,1\}\right)
$$

as an $h$-adic topological $R$-module. Hence we see that the $R$-module homomorphism $\tilde{U}_{h}^{\sigma} \rightarrow V\left(s \rightarrow x \cdot 1_{V}\right)$ is an isomorphism. This completes the proof.

Defining the coproduct $\Delta$, the antipode $S$ and the counit $\varepsilon$ by

$$
\begin{aligned}
& \Delta\left(E_{i}\right)=E_{i} \otimes 1+\exp \left(h H_{\alpha_{i}}\right) \cdot \sigma^{p\left(\alpha_{i}\right)} \otimes E_{i}, \\
& \Delta\left(F_{i}\right)=F_{i} \otimes \exp \left(-h H_{\alpha_{i}}\right)+\sigma^{p\left(\alpha_{i}\right)} \otimes F_{i}, \\
& \Delta(H)=H \otimes 1+1 \otimes H(H \in \mathscr{H}), \Delta(\sigma)=\sigma \otimes \sigma, \\
& S\left(E_{i}\right)=-\exp \left(-h H_{\alpha_{i}}\right) \cdot \sigma^{p\left(\alpha_{i}\right)} E_{i}, S\left(F_{i}\right)=-F_{i} \exp \left(h H_{\alpha_{i}}\right) \cdot \sigma^{p\left(\alpha_{i}\right)}, \\
& S(H)=-H(H \in \mathscr{H}), S(\sigma)=\sigma, \\
& \varepsilon\left(E_{i}\right)=\varepsilon\left(F_{i}\right)=\varepsilon(H)=0, \varepsilon(\sigma)=1,
\end{aligned}
$$


the algebra $\tilde{U}_{h}^{\sigma}$ becomes a Hopf algebra.

2.2. Let $\left(\mathscr{E}, \Pi=\left\{\alpha_{1}, \cdots, \alpha_{n}\right\}, p\right)$ be a triple system. Put $R^{\prime}=C[[\sqrt{h}]]$. Then $R^{\prime}=R \oplus \sqrt{h} R$. Let $\widetilde{U}_{\sqrt{h}}^{\prime} b_{+}^{\sigma}=\widetilde{U}_{\sqrt{h}}^{\prime} b_{+}^{\sigma}(\mathscr{E}, \Pi, p)$ be a $\sqrt{h}$-adic topological $R$-algebra with generators $E_{i}(1 \leq i \leq n), H^{\prime} \in \mathscr{H}, \sigma$ and relations: (In $\tilde{U}_{\sqrt{h}}^{\prime} b_{+}^{\sigma}$, we write $H^{\prime}$ for $H \in \mathscr{H}$ ).

(2.2.1) $\sigma^{2}=1, \sigma H^{\prime} \sigma=H^{\prime}\left(H^{\prime} \in \mathscr{H}\right), \sigma E_{i} \sigma=(-1)^{p\left(\alpha_{i}\right)} E_{i}$,

(2.2.2) $\left[H_{1}^{\prime}, H_{2}^{\prime}\right]=0\left(H_{1}^{\prime}, H_{2}^{\prime} \in \mathscr{H}\right)$,

(2.2.3) $\left[H^{\prime}, E_{i}\right]=\sqrt{h} \alpha_{i}\left(H^{\prime}\right) E_{i}\left(H^{\prime} \in \mathscr{H}\right)$.

A topological Hopf algebra structure of $\widetilde{U}_{\sqrt{h}}^{\prime} b_{+}^{\sigma}$ introduced by defining the coproduct $\Delta^{\prime}$, the antipode $S^{\prime}$ and the counit $\varepsilon^{\prime}$ is defined as follows: (Here $H^{\prime}$ denotes an arbitrary element of $\mathscr{H}$.)

$$
\begin{aligned}
& \Delta^{\prime}\left(E_{i}\right)=E_{i} \otimes 1+\exp \left(\sqrt{h} H_{\alpha_{i}}^{\prime}\right) \cdot \sigma^{p\left(\alpha_{2}\right)} \otimes E_{i}, \\
& \Delta^{\prime}\left(H^{\prime}\right)=H^{\prime} \otimes 1+1 \otimes H^{\prime}\left(H^{\prime} \in \mathscr{H}\right), \Delta^{\prime}(\sigma)=\sigma \otimes \sigma
\end{aligned}
$$

(2.2.5) $S^{\prime}\left(E_{i}\right)=-\exp \left(\sqrt{h} H_{\alpha_{i}}^{\prime}\right) \cdot \sigma^{p\left(\alpha_{2}\right)} E_{i}$,

$$
S^{\prime}\left(H^{\prime}\right)=-H^{\prime}\left(H^{\prime} \in \mathscr{H}\right), S^{\prime}(\sigma)=\sigma \text {, }
$$

$\varepsilon^{\prime}\left(E_{i}\right)=\varepsilon^{\prime}\left(F_{i}\right)=\varepsilon^{\prime}\left(H^{\prime}\right)=0, \varepsilon^{\prime}(\sigma)=1$

Let $\tilde{\Omega}_{+}^{\prime}: \tilde{U}_{\sqrt{h}}^{\prime} b_{+}^{\sigma} \rightarrow R^{\prime} \otimes \tilde{U}_{h}^{\sigma}$ be a continuous $R^{\prime}$-algebra homomorphism defined by putting $\tilde{\Omega}_{+}^{\prime}\left(E_{i}\right)=E_{i}, \tilde{\Omega}_{+}^{\prime}\left(H^{\prime}\right)=\sqrt{h} H^{\prime}\left(H^{\prime} \in \mathscr{H}\right), \tilde{\Omega}_{+}^{\prime}(\sigma)=\sigma$. Then $\tilde{\Omega}_{+}^{\prime}$ is a $\sqrt{h}$-adic topological Hopf algebra homomorphism. Similarly to the proof of Lemma 2.1.4, we have:

Lemma 2.2.7. (i) $\tilde{\Omega}_{+}^{\prime}$ is injective. (ii) Put $\tilde{N}_{+}^{\prime}=R^{\prime} \otimes \tilde{N}_{+}=R^{\prime}\left\langle E_{1}, \cdots, E_{n}\right\rangle$, $\subseteq\left[\mathscr{H}^{R^{\prime}}\right]=R^{\prime} \otimes \subseteq\left[\mathscr{H}^{R}\right]$ and $R^{\prime}\langle\sigma\rangle=R^{\prime} \otimes R^{\prime}\langle\sigma\rangle$. Then we have an isomorphism of $\sqrt{h}$-adic topological $R^{\prime}$-modules:

$$
\tilde{N}_{+}^{\prime} \hat{\otimes} \subseteq\left[\mathscr{H}^{R^{\prime}}\right] \otimes R^{\prime}\langle\sigma\rangle \rightarrow \widetilde{U}_{\sqrt{h}}^{\prime} b_{+}^{\sigma}\left(X \otimes Z^{\prime} \otimes \sigma^{c} \rightarrow X \cdot Z^{\prime} \cdot \sigma^{c}(c=0,1)\right)
$$

In particular, if $H_{1}^{\prime}, \cdots, H_{N}^{\prime}$ are $C$-basis of $\mathscr{H}$, the elements 
$(2.2 .8)$

$$
E_{i_{1}} \cdots E_{i_{u}} \cdot H_{1}^{\prime a_{1}} \cdots H_{N}^{\prime a_{N}} \cdot \sigma^{c}\left(1 \leq i_{1}, \cdots, i_{u} \leq n, a_{1}, \cdots, a_{N} \in \mathbb{Z}_{+}, c \in\{0,1\}\right)
$$

form a topological basis of $\widetilde{U}_{\sqrt{h}}^{\prime} b_{+}^{\sigma}$.

2.3. For $a \in \mathbb{Z}_{+}$, let $\mathfrak{S}_{a}^{\prime}$ be the submodule of homogeneous elements of degree $a$ of $\mathfrak{S}\left[\mathscr{H}^{R^{\prime}}\right] . \quad$ Then $\mathfrak{S}\left[\mathscr{H}^{R^{\prime}}\right]=\bigoplus_{a \in \mathbb{Z}_{+}} \mathfrak{S}_{a}^{\prime}$. Put $\mathbb{P}_{+}=\mathbb{Z}_{+} \alpha_{1} \oplus \cdots \oplus \mathbb{Z}_{+} \alpha_{n}$ $\in \mathscr{E}$. For $\lambda \in P_{+}$, we put $\tilde{N}_{+, \lambda}^{\prime}=\underset{\alpha_{i 1}+\cdots+\alpha_{i_{v}}=\lambda}{\bigoplus} R^{\prime} E_{i_{1}} \cdots E_{i_{v}} . \quad$ Then $\tilde{N}_{+}^{\prime}=\bigoplus_{\lambda \in P_{+}} \tilde{N}_{+, \lambda}^{\prime}$.

We define the elements $H_{\lambda}^{\prime \circ}(\lambda \in \mathscr{E}), E_{i}^{\circ}(1 \leq i \leq n), \sigma^{\circ} \in\left(\tilde{U}_{\sqrt{h}}^{\prime} b_{+}^{\sigma}\right)^{*}$ as follows: (Here $X$ (resp. $Z^{\prime}$ ) denotes a non-zero element of $\tilde{N}_{+, \mu}^{\prime}$ (resp. $\mathfrak{S}_{a}^{\prime}$ ) and $c \in\{0,1\}$.)

$$
\begin{aligned}
& H_{\lambda}^{\prime \circ}\left(X \cdot Z^{\prime} \cdot \sigma^{c}\right)=\left\{\begin{array}{lll}
\lambda\left(Z^{\prime}\right) & \text { if } X=1 & \text { and } a=1 \\
0 & \text { if } \mu \neq 0 \text { or } a \neq 1,
\end{array}\right. \\
& \text { (2.3.2) } E_{i}^{\circ}\left(X \cdot Z^{\prime} \cdot \sigma^{c}\right)=\left\{\begin{array}{lll}
1 & \text { if } X=E_{i} \text { and } Z^{\prime}=1, \\
0 & \text { if } \mu \neq \alpha_{i} \text { or } a \neq 0,
\end{array}\right. \\
& \sigma^{\circ}\left(X \cdot Z^{\prime} \cdot \sigma^{c}\right)=\left\{\begin{array}{l}
(-1)^{c} \text { if } X=Z^{\prime}=1 \\
0 \text { if } \mu \neq 0 \text { or } a \neq 0 .
\end{array}\right.
\end{aligned}
$$

Lemma 2.3.4. (i) $E_{i}^{\circ}, H_{\lambda}^{\prime \circ}, \sigma^{\circ} \in\left(\tilde{U}_{\sqrt{h}}^{\prime} b_{+}^{\sigma}\right)^{\circ}$.

(ii) There is a topological $R^{\prime}$-Hopf algebra homomorphism $\Theta: \widetilde{U}_{\sqrt{h}}^{\prime} b_{+}^{\sigma} \rightarrow$ $\overline{\left(\widetilde{U}_{\sqrt{h}}^{\prime} b_{+}^{\sigma}\right)^{\circ}}$ such that $\Theta\left(H_{\lambda}^{\prime}\right)=H_{\lambda}^{\prime \circ}, \Theta\left(E_{i}\right)=E_{i}^{\circ}, \Theta(\sigma)=\sigma^{\circ}$.

Proof. (i) We show $E_{i}^{\circ} \in\left(\tilde{U}_{\sqrt{h}}^{\prime} b_{+}^{\sigma}\right)^{\circ}$ only. It can be easily shown that the basis elements $x$ of (2.2.8) satisfying $x \cdot E_{i}^{\circ} \neq 0$ are $1, \sigma, E_{i}$ and $E_{i} \sigma$. Hence we have $\operatorname{rank}\left(\tilde{U}_{\sqrt{h}}^{\prime} b_{+}^{\sigma}\right) \cdot E_{i}^{\circ} \leq 4$, which implies $E_{i}^{\circ} \in\left(\tilde{U}_{\sqrt{h}}^{\prime} b_{+}^{\sigma}\right)^{\circ}$. Similarly, we have $H_{\lambda}^{\circ}, \sigma^{\circ} \in\left(\widetilde{U}_{\sqrt{h}}^{\prime} b_{+}^{\sigma}\right)^{\circ}$.

(ii) First we show that $\Theta$ is an algebra map. We show that the elements $E_{i}^{\circ}, H_{\lambda}^{\prime \circ}$ satisfy (2.2.3). By the definition of the coproduct $\Delta^{\prime}$ and the definitions of $E_{i}^{\circ}, H_{\lambda}^{\prime \circ}, \sigma^{\circ}$, for the basis elements $x$ of $(2.2 .8)$, we have:

$$
E_{i}^{\circ} \cdot H_{\lambda}^{\circ}(x)=\left\{\begin{array}{l}
(\lambda, \mu) \text { if } x=E_{i} \cdot H_{\mu}^{\prime} \cdot \sigma^{c}, \\
0 \text { otherwise, }
\end{array}\right.
$$




$$
H_{\lambda}^{\prime \circ} \cdot E_{i}^{\circ}(x)=\left\{\begin{array}{l}
\sqrt{h}\left(\lambda, \alpha_{i}\right) \text { if } x=E_{i} \cdot \sigma^{c} \\
(\lambda, \mu) \text { if } x=E_{i} \cdot H_{\mu}^{\prime} \sigma \\
0 \quad \text { otherwise. }
\end{array}\right.
$$

Hence it follows that $H_{\lambda}^{\prime \circ} \cdot E_{i}^{\circ}=E_{i}^{\circ} H_{\lambda}^{\circ \circ}+\sqrt{h}\left(\lambda, \alpha_{i}\right) E_{i}^{\circ}$, which is the relation (2.2.3). Similarly, we can show that the elements $E_{i}^{\circ}, H_{\lambda}^{\prime \circ}, \sigma^{\circ}$ satisfy the relations $(2.2 .1-2)$.

Next we show that $\Theta$ is a Hopf algebra map. Let $m$ denote the multiplication of $\tilde{U}_{\sqrt{h}}^{\prime} b_{+}^{\sigma}$. We are going to show that

$$
{ }^{t} m\left(E_{i}^{\circ}\right)=E_{i}^{\circ} \otimes \varepsilon^{\prime}+\exp \left(\sqrt{h} H_{\alpha_{i}}^{\prime \circ}\right) \cdot\left(\sigma^{\circ}\right)^{p\left(\alpha_{i}\right)} \otimes E_{i}^{\circ}
$$

which is the one of (2.2.4). Let $x$ and $y$ be a pair of the basis elements (2.2.8). By (2.2.1-13), we have:

$$
\begin{aligned}
& { }^{t} m\left(E_{i}^{\circ}\right)(x \otimes y) \\
& =\left\{\begin{array}{l}
1 \quad \text { if } x=E_{i} \sigma^{c}(c=0,1) \text { and } y=\sigma^{d}(d=0,1), \\
(-1)^{p\left(\alpha_{i}\right) c} \cdot(\sqrt{h})^{a_{1}+\cdots+a_{N}} \cdot \alpha_{i}\left(H_{1}^{\prime}\right)^{a_{1} \cdots \alpha_{i}\left(H_{N}^{\prime}\right)^{a_{N}}} \\
\text { if } x=H_{1}^{\prime} a_{1} \cdots H_{N}^{\prime a_{N}} \sigma^{c}\left(a_{1}, \cdots, a_{N} \in Z_{+}, c=0,1\right), y=E_{i} \sigma^{d}, \\
0 \quad \text { otherwise. }
\end{array}\right.
\end{aligned}
$$

Here we note that, for the basis elements $x$ of (2.2.8),

$$
\begin{aligned}
& \left(H_{\lambda}^{\prime \circ}\right)^{a} \cdot\left(\sigma^{\circ}\right)^{c}(x) \\
& = \begin{cases}a ! \lambda\left(H_{i_{1}}^{\prime}\right) \cdots \lambda\left(H_{i_{a}}^{\prime}\right)(-1)^{c d} & \text { if } \quad x=H_{i_{i}}^{\prime} \cdots H_{i_{a}}^{\prime} \cdot \sigma^{d} \\
0 & \quad\left(1 \leq i_{1} \leq \cdots \leq i_{a} \leq N, d=0,1\right), \\
0 & \text { otherwise. }\end{cases}
\end{aligned}
$$

Hence we have:

$$
{ }^{t} m\left(E_{i}^{\circ}\right)=E_{i}^{\circ} \otimes \varepsilon^{\prime}+\sum_{a \in \mathbb{Z}_{+}} \frac{1}{a !}(\sqrt{h})^{a}\left(H_{\alpha_{1}}^{\prime \circ}\right)^{a} \cdot\left(\sigma^{\circ}\right)^{p\left(\alpha_{i}\right)} \otimes E_{i}^{\circ}
$$

in $\overline{\left(\widetilde{U}_{\sqrt{h}}^{\prime} b_{+}^{\sigma}\right)^{\circ}} \hat{\otimes} \overline{\left(\widetilde{U}_{\sqrt{h}}^{\prime} b_{+}^{\sigma}\right)^{\circ}}$. The above is nothing else but (2.3.5). Similarly, we can prove that, $E_{i}^{\circ}, H_{\lambda}^{\circ}, \sigma^{\circ}$ satisfy $(2.2 .4-6)$. This completes the proof. 
2.4. We define a symmetric topological $R^{\prime}$-Hopf algebra pairing $\langle$,$\rangle :$ $\widetilde{U}_{\sqrt{h}}^{\prime} b_{+}^{\sigma} \times{\widetilde{U^{h}}}_{\sqrt{h}}^{\prime} b_{+}^{\sigma} \rightarrow R^{\prime}$ by $\langle x, y\rangle=\Theta(x)(y) . \quad$ By $(2.3 .1-3)$ and (2.3.6), we have:

Lemma 2.4.1. Let $\varepsilon_{i}(1 \leq i \leq N)$ be a C-basis of $\mathscr{E}$ such that $\left(\varepsilon_{i}, \varepsilon_{j}\right)=\delta_{i j}$. Let $X \in \tilde{N}_{+, \lambda}^{\prime}$ and $Y \in \tilde{N}_{+, \mu}^{\prime}$. Then we have:

$$
\begin{aligned}
\left\langle X \cdot H_{\varepsilon_{1}}^{\prime a_{1}} \cdots H_{\varepsilon_{N}}^{\prime a_{N}} \cdot \sigma^{c}, Y \cdot H_{\varepsilon_{1}}^{\prime b_{1}} \cdots H_{\varepsilon_{N}}^{\prime b_{N}} \cdot \sigma^{d}\right\rangle \\
=(-1)^{c d} \cdot\left(\prod_{i=1}^{N}\left(\delta_{a_{i} b_{i}} \cdot a_{i} !\right)\right) \cdot \delta_{\lambda \mu}\langle X, Y\rangle .
\end{aligned}
$$

2.5. Let $\tilde{\mathfrak{D}}^{\prime}=D\left(\widetilde{U}_{\sqrt{h}}^{\prime} b_{+}^{\sigma},\left(\widetilde{U}_{\sqrt{h}}^{\prime} b_{+}^{\sigma}\right)^{o p}\right)$ be the quantum double defined in $\S 1$. Then $\tilde{\mathfrak{D}}^{\prime} \simeq \widetilde{U}_{\sqrt{h}}^{\prime} b_{+}^{\sigma} \hat{\otimes} \tilde{U}_{\sqrt{h}}^{\prime} b_{+}^{\sigma}$ as $R^{\prime}$-modules. For an element $X \in$ $\widetilde{U}_{\sqrt{h}}^{\prime} b_{+}$, we write $X$ for $X \otimes 1$ and $X^{\circ}$ for $1 \otimes X$.

Lemma 2.5.1. The h-adic topological $R^{\prime}$-algebra $\tilde{\mathfrak{D}^{\prime}}$ is $h$-adically defined with the generators $E_{i}, E_{i}^{\circ}(1 \leq i \leq n) H, H^{\circ}\left(H^{\prime} \in \mathscr{H}\right), \sigma, \sigma^{\circ}$ and the relations:

(2.5.2) The elements $\sigma, \sigma^{\circ}, H^{\prime}, H^{\circ}$ are mutually commutative.

(2.5.3) $\sigma E_{i} \sigma=(-1)^{p\left(\alpha_{i}\right)} E_{i}, \sigma E_{i}^{\circ} \sigma=(-1)^{p\left(\alpha_{i}\right)} E_{i}^{\circ}$, $\sigma^{\circ} E_{i} \sigma^{\circ}=(-1)^{p\left(\alpha_{i}\right)} E_{i}, \sigma^{\circ} E_{i}^{\circ} \sigma^{\circ}=(-1)^{p\left(\alpha_{i}\right)} E_{i}^{\circ}$,

(2.5.4) $\left[H^{\prime}, E_{i}\right]=\sqrt{h} \alpha_{i}\left(H^{\prime}\right) E_{i} .\left[H^{\circ}, E_{i}\right]=-\sqrt{h} \alpha_{i}\left(H^{\circ}\right) E_{i}$, $\left[H^{\prime \circ}, E_{i}^{\circ}\right]=\sqrt{h} \alpha_{i}\left(H^{\prime \circ}\right) E_{i}^{\circ} .\left[H^{\prime}, E_{i}^{\circ}\right]=-\sqrt{h} \alpha_{i}\left(H^{\prime}\right) E_{i}^{\circ}$,

$$
E_{i} E_{j}^{\circ}-E_{j}^{\circ} E_{i}=\delta_{i j}\left(\exp \left(\sqrt{h} H_{\alpha_{i}}^{\prime \circ}\right) \sigma^{\circ p\left(\alpha_{i}\right)}-\exp \left(\sqrt{h} H_{\alpha_{i}}^{\prime}\right) \sigma^{p\left(\alpha_{i}\right)}\right)
$$

Proof. Put $L_{i}=\exp \left(\sqrt{h} H_{\alpha_{i}}^{\prime}\right) \sigma^{p\left(\alpha_{i}\right)}$ and $L_{i}^{\circ}=\exp \left(\sqrt{h} H_{\alpha_{i}}^{\prime}\right) \sigma^{p\left(\alpha_{i}\right)}$. First we show that (2.5.5) holds in $\tilde{\mathfrak{D}}^{\prime}$. By Proposition 1.4.1 and (2.3.1-3), we have:

$$
\begin{aligned}
E_{j}^{\circ} E_{i}= & \Phi\left(E_{j}^{\circ} \otimes E_{i}\right) \\
= & \left\langle L_{j}^{\circ}, 1\right\rangle\left\langle 1, S^{\prime-1}\left(L_{i}\right)\right\rangle E_{i} E_{j}^{\circ}+\left\langle E_{j}^{\circ}, E_{i}\right\rangle\left\langle 1, S^{\prime-1}\left(L_{i}\right)\right\rangle L_{i} \\
& +\left\langle L_{j}^{\circ}, L_{i}\right\rangle\left\langle E_{j}^{\circ}, S^{\prime-1}\left(E_{i}\right)\right\rangle L_{j}^{\circ} \\
= & -E_{i} E_{j}^{\circ}+\delta_{i j} L_{i}-\delta_{i j} L_{j}^{\circ} .
\end{aligned}
$$

Similarly, we can show (2.5.2-4). On the other hand, it can be easily shown that the $R^{\prime}$-algebra defined by the relations $(2.5 .2-5)$ is generated by the elements 


$$
E_{i_{1}} \cdots E_{i_{u}} H_{1}^{\prime a_{1}} \cdots H_{N}^{\prime a_{N}} \sigma^{c} \cdot E_{j_{1}}^{\circ} \cdots E_{j_{v}}^{\circ} H_{1}^{\prime \circ b_{1}} \cdots H_{N}^{\prime o b_{N}} \sigma^{\alpha}
$$

as an $R^{\prime}$-module where $\left\{H_{1}^{\prime}, \cdots, H_{N}^{\prime}\right\}$ is a basis of $\mathscr{H}$ and $1 \leq i_{1}, \cdots, i_{u}, j_{1}, \cdots, j_{v} \leq N$, $a_{1}, \cdots, a_{N}, b_{1}, \cdots, b_{N} \in Z_{+}, c, d \in\{0,1\}$. Since $\tilde{\mathfrak{D}}^{\prime} \simeq \tilde{U}_{\sqrt{h}}^{\prime} b_{+}^{\sigma} \otimes \tilde{U}_{\sqrt{h}}^{\prime} b_{+}^{\sigma}$, this completes the proof.

2.6. Let $I_{b_{+}}^{\prime}\left(\subset \widetilde{U}_{\sqrt{h}}^{\prime} b_{+}^{\sigma}\right)$ (resp. $I_{+}^{\prime}\left(\subset \widetilde{N}_{+}^{\prime}\right)$ ) be the kernel of $\langle$, $\rangle$ (resp. $\left.\langle,\rangle_{\mid \tilde{N}^{\prime}+\times \tilde{N}^{\prime}}\right)$. The following lemma is useful.

Lemma 2.6.1. If $J^{\prime}$ is a bi-ideal of $\tilde{U}_{\sqrt{h}}^{\prime} b_{+}^{\sigma}$ such that, $J^{\prime} \subset \sum_{i, j} E_{i} E_{j} \widetilde{U}_{\sqrt{h}}^{\prime} b_{+}^{\sigma}$, then $J^{\prime} \subset I_{b_{+}}^{\prime}$.

Proof. By (2.3.1-3), the generators $E_{i}(1 \leq i \leq n), H(H \in \mathscr{H}), \sigma$ are orthogonal to $J^{\prime}$. Hence the lemma follows.

2.7. Put $U_{\sqrt{h}}^{\prime} b_{+}^{\sigma}=\tilde{U}_{\sqrt{h}}^{\prime} b_{+}^{\sigma} / I_{b_{+}}^{\prime}$ and $N_{+}^{\prime}=\tilde{N}_{+}^{\prime} / I_{+}^{\prime}$. Put $I_{+, \lambda}^{\prime}=I_{+}^{\prime} \cap \tilde{N}_{+, \lambda}^{\prime}$ and $N_{+, \lambda}^{\prime}=\tilde{N}_{+, \lambda}^{\prime} / I_{+, \lambda}^{\prime}$ for $\lambda \in P_{+}$.

Lemma 2.7.1. (i) $N_{+, \lambda}^{\prime}$ is a free $R^{\prime}$-module of finite rank. $\quad N_{+}^{\prime}=\bigoplus_{\lambda \in P_{+}} N_{+, \lambda}^{\prime}$. (ii) $I_{b_{+}}^{\prime}=I_{+}^{\prime} \Im\left[\mathscr{H}^{R^{\prime}}\right] \cdot R^{\prime}\langle\sigma\rangle$. (iii) As topological $R^{\prime}$-modules, $U_{\sqrt{h}}^{\prime} b_{+}^{\sigma} \simeq N_{+}^{\prime} \hat{\otimes}$ $\mathfrak{S}\left[\mathscr{H}^{R^{\prime}}\right] \otimes R^{\prime}\langle\sigma\rangle\left(X \cdot Z^{\prime} \cdot \sigma^{c} \leftarrow X \otimes Z^{\prime} \otimes \sigma^{c}\right)$. In particular, $U_{\sqrt{h}}^{\prime} b_{+}^{\sigma}$ is a topologically free $R^{\prime}$-Hopf algebra.

Proof. Let $c \in R^{\prime} \backslash\{0\}$ and $x \in \tilde{N}_{+, \lambda}^{\prime}$. If $c x \in I_{+, \lambda}^{\prime}$, then $c\langle x, y\rangle=\langle c x, y\rangle=0$ for all $y \in \tilde{N}_{+}$. Hence $\langle x, y\rangle=0$ which implies $x \in I_{+, \lambda}^{\prime}$. Hence the freeness of $N_{+, \lambda}^{\prime}$ follows.

From Lemma 2.4.1, we obtain:

$$
\langle,\rangle \simeq\langle,\rangle_{\mid \tilde{N}^{\prime}+\otimes \tilde{N}^{\prime}+} \otimes\langle,\rangle_{\mid \Subset\left[\mathscr { H } ^ { R ^ { \prime } ] } \otimes \Subset \left[\mathscr{H}^{\left.R^{\prime}\right]}\right.\right.} \otimes\langle,\rangle_{\mid R^{\prime}\langle\sigma\rangle \otimes R^{\prime}\langle\sigma\rangle}
$$

and $\tilde{N}_{+}^{\prime}=\underset{\lambda \in P_{+}}{\oplus} \tilde{N}_{+, \lambda}^{\prime}$ (Here $\stackrel{\perp}{\oplus}$ denotes the orthogonal direct sum). Then we have (i) and (ii). We immediately obtain (iii) from (ii). 
2.8. Let $D^{\prime}=D\left(U_{\sqrt{h}}^{\prime} b_{+},\left(U_{\sqrt{h}}^{\prime} b_{+}\right)^{o p}\right)\left(\simeq U_{\sqrt{h}}^{\prime} b_{+} \hat{\otimes}\left(U_{\sqrt{h}}^{\prime} b_{+}\right)^{o p}\right)$ be the quantum double defined in $\S 1$. For an element $X \in U_{\sqrt{h}}^{\prime} b_{+}^{\sigma}$, we write $X$ for $X \otimes 1 \in \mathfrak{D}^{\prime}$ and $X^{\circ}$ for $1 \otimes X \in \mathfrak{D}^{\prime}$. For a subset $M$ of $U_{\sqrt{h}}^{\prime} b_{+}$(resp. $\left.\widetilde{U}_{\sqrt{h}}^{\prime} b_{+}\right)$, we write $M$ for $\left\{m \otimes 1 \in \mathfrak{D}^{\prime}\right.$ (resp. $\left.\left.\tilde{D}^{\prime}\right) \mid m \in M\right\}$ and $M^{\circ}$ for $\left\{1 \otimes m \in \mathfrak{D}^{\prime}\right.$ (resp. $\left.\left.\tilde{\mathfrak{D}}^{\prime}\right) \mid m \in M\right\}$.

By the definitions of the quantum doubles $\tilde{D^{\prime}}$ and $\mathfrak{D}^{\prime}$ (see Proposition 1.4.1), we have:

Lemma 2.8.1. (i) As $R^{\prime}$-modules,

$$
\begin{aligned}
& \tilde{\mathfrak{D}}^{\prime} \simeq \tilde{N}^{\prime} \hat{\otimes} \mathfrak{S}\left[\mathscr{H}^{R^{\prime}}\right] \hat{\otimes} R^{\prime}\langle\sigma\rangle \hat{\otimes} \tilde{N}^{\prime \circ} \hat{\otimes} \mathfrak{S}\left[\mathscr{H}^{R^{\prime}}\right]^{\circ} \hat{\otimes} R^{\prime}\left\langle\sigma^{\circ}\right\rangle, \\
& D^{\prime} \simeq N^{\prime} \otimes \widehat{\Theta}\left[\mathscr{H}^{R^{\prime}}\right] \hat{\otimes} R^{\prime}\langle\sigma\rangle \hat{\otimes} N^{\prime \circ} \hat{\otimes} \subseteq\left[\mathscr{H}^{R^{\prime}}\right]^{\circ} \hat{\otimes} R^{\prime}\left\langle\sigma^{\circ}\right\rangle .
\end{aligned}
$$

(ii) Let $\Psi: \tilde{D}^{\prime} \rightarrow \mathfrak{D}^{\prime}$ be a natural epimorphism defined by $\Psi(\tilde{X} \otimes \tilde{Y})=$ $X \otimes Y$ where $X=\tilde{X}+I_{b_{+}}^{\prime}$ and $Y=\tilde{Y}+I_{b_{+}}^{\prime}$. Then

$$
\operatorname{Ker} \Psi=I_{+}^{\prime} \cdot \mathfrak{S}\left[\mathscr{H}^{R^{\prime}}\right] \cdot R^{\prime}\langle\sigma\rangle \hat{\otimes}\left({\tilde{U^{\prime h}}}_{\sqrt{h}}^{\prime} b_{+}^{\sigma}\right)^{\circ}+\tilde{U}_{\sqrt{h}}^{\prime} b_{+}^{\sigma} \hat{\otimes} I_{+}^{\prime \circ} \cdot \mathfrak{S}\left[\mathscr{H}^{R^{\prime}}\right]^{\circ} R^{\prime}\left\langle\sigma^{\circ}\right\rangle
$$

2.9. Let $N_{+}$(resp. $\tilde{N}_{+}$) be the unital R-subalgebra of $N_{+}^{\prime}$ (resp. $\tilde{N}_{+}^{\prime}$ ) generated by the elements $E_{i}(1 \leq i \leq n)$. Since $N_{+}^{\prime}$ is free (see 2.7.1), $N_{+}$ is a free $R$-module. Let $I_{+}=I_{+}^{\prime} \cap \tilde{N}_{+}$. For $\lambda \in P_{+}$, we put $\tilde{N}_{+, \lambda}=\tilde{N}_{+, \lambda}^{\prime} \cap \tilde{N}_{+}$, $N_{+, \lambda}=N_{+, \lambda}^{\prime} \cap N_{+}$and $I_{+, \lambda}=I_{+, \lambda}^{\prime} \cap N_{+}$.

Lemma 2.9.1. (i)

$$
\begin{aligned}
& N_{+}^{\prime}=N_{+} \oplus \sqrt{h} N_{+}, I_{+}^{\prime}=I_{+} \oplus \sqrt{h} I_{+}, N_{+}=\tilde{N}_{+} / I_{+}, \\
& N_{+, \lambda}^{\prime}=N_{+, \lambda} \oplus \sqrt{h} N_{+, \lambda}, I_{+, \lambda}^{\prime}=I_{+, \lambda} \oplus \sqrt{h} I_{+, \lambda}, \\
& N_{+, \lambda}=\tilde{N}_{+, \lambda} / I_{+, \lambda} .
\end{aligned}
$$

where $\lambda \in P_{+}$.

(ii) For $\lambda \in P_{+}$, there exists a free $R$-module $L_{+, \lambda}$ such that $\tilde{N}_{+, \lambda}=$ $I_{+, \lambda} \oplus L_{+, \lambda}$.

Proof. (i) We have $I_{+, \lambda}^{\prime}=I_{+, \lambda} \oplus \sqrt{h} I_{+, \lambda}$, since $\langle X, Y\rangle \in R$ for all $X, Y \in \tilde{N}_{+}$. The rests follow easily from this.

(ii) By Lemma 2.7.1, $N_{+. \lambda}^{\prime}$ is a free $R^{\prime}$-module of finite rank. Hence, by (i), $N_{+, \lambda}^{\prime}$ is a free $R$-module of finite rank. Since $N_{+, \lambda}=\tilde{N}_{+, \lambda} / I_{+, \lambda}$, 
choosing representatives $x_{1}, \cdots, x_{u} \in \tilde{N}_{+, \lambda}$ such that $x_{1}, \cdots, x_{u}$ form a basis of $N_{+, \lambda}$ (modulo $I_{+, \lambda}$ ), we have $\tilde{N}_{+, \lambda}=I_{+, \lambda} \oplus\left(R x_{1} \oplus \cdots \oplus R x_{u}\right)$. Hence, putting, $L_{+, \lambda}=R x_{1} \oplus \cdots \oplus R x_{u}$ the part (ii) follows.

Lemma 2.9.2. Put $K_{i}=\exp \left(\sqrt{h} H_{\alpha_{i}}^{\prime}\right), K_{i}^{\circ}=\exp \left(\sqrt{h} H_{\alpha_{i}}^{\circ}\right) \in \mathfrak{D}^{\prime}(1 \leq i \leq n)$. Let $\boldsymbol{T}$ (resp. $\boldsymbol{T}^{\circ}$ ) be the $R$-subalgebra of $\mathfrak{D}^{\prime}$ generated by the elements $K_{i}^{ \pm 1}$ (resp. $\left.K_{i}^{\circ \pm 1}\right)(1 \leq i \leq n)$. Let $U$ be the $R$-subalgebra of $\mathfrak{D}^{\prime}$ algebraically generated by the elements $\sigma^{ \pm 1}, \sigma^{\circ \pm 1}$ and $K_{i}^{ \pm 1}, K_{i}^{\circ \pm 1}, E_{i}, E_{i}^{\circ}(1 \leq i \leq n)$. Then

(i) $U$ is a (non-topological) $R$-Hopf subalgebra of $\mathfrak{D}^{\prime}$.

(ii) As R-modules, $U \simeq N_{+} \otimes T \otimes R\langle\sigma\rangle \otimes N_{+}^{\circ} \otimes T^{\circ} \otimes R\left\langle\sigma^{\circ}\right\rangle$ $\left(X t \sigma^{c} \quad Y^{\circ} s^{\circ} \sigma^{o d} \leftarrow X \otimes t \otimes \sigma^{c} \otimes Y^{\circ} \otimes s^{\circ} \otimes \sigma^{\circ d}\right)\left(X \in N_{+}, t \in T, c \in\{0,1\}, Y^{\circ} \in\right.$ $\left.N_{+}^{\circ}, s^{\circ} \in T^{\circ}, d \in\{0,1\}\right)$. The elements $K_{1}^{l_{1}} \cdots K_{n}^{l_{n}}\left(\right.$ resp. $\left.K_{1}^{\circ l_{1}} \cdots K_{n}^{\circ l_{n}}\right)\left(l_{1}, \cdots, l_{n} \in Z\right)$ form an R-basis of $\boldsymbol{T}$ (resp. $\boldsymbol{T}^{\circ}$ ).

Proof. (i) By (2.2.4-6) and Proposition 1.4.1, we have (i).

(ii) By (2.2.1-3), (2.5.2-5), we have

$$
\boldsymbol{U}=N_{+} \cdot \boldsymbol{T} \cdot R\langle\sigma\rangle \cdot N_{+}^{\circ} \cdot \boldsymbol{T}^{\circ} \cdot R\left\langle\sigma^{\circ}\right\rangle
$$

By Lemma 2.8.1 (i), we can easily show that

$$
\begin{aligned}
N_{+} \cdot K_{1}^{l_{1}} \cdots K_{n}^{l_{n}} \cdot R\langle\sigma\rangle \cdot N_{+}^{\circ} \cdot K_{1}^{\mathrm{om}} \cdots K_{n}^{\circ m_{n}} \cdot R\left\langle\sigma^{\circ}\right\rangle \\
\simeq N_{+} \otimes K_{1}^{l_{1}} \cdots K_{n}^{l_{n}} \otimes R\langle\sigma\rangle \otimes N_{+}^{\circ} \otimes K_{1}^{\circ m_{1}} \cdots K_{n}^{\circ m_{n}} \otimes R\left\langle\sigma^{\circ}\right\rangle
\end{aligned}
$$

holds in $\mathfrak{D}^{\prime}$ for each $l_{1}, \cdots, l_{n}, m_{1}, \cdots, m_{n} \in \boldsymbol{Z}$. Therefore, it is enough to show

$$
U \simeq \bigoplus_{l_{i}, m_{1} \in Z} N_{+} \cdot K_{1}^{l_{1}} \cdots K_{n}^{l_{n}} \cdot R\langle\sigma\rangle \cdot N_{+}^{\circ} \cdot K_{1}^{\circ m_{1}} \cdots K_{n}^{\circ m_{n}} \cdot R\left\langle\sigma^{\circ}\right\rangle
$$

Take $\alpha_{n+1}, \cdots, \alpha_{N} \in \mathscr{E}$ such that $\alpha_{1}, \cdots, \alpha_{n}, \alpha_{n+1}, \cdots, \alpha_{N}$ form a basis of $\mathscr{E}$. For $1 \leq i \leq n$ define $R^{\prime}$-module maps $\partial_{i}: \mathfrak{D}^{\prime} \rightarrow \mathfrak{D}^{\prime}$ and $\partial_{i}^{\circ}: \mathfrak{D}^{\prime} \rightarrow \mathfrak{D}^{\prime}$ by:

$$
\begin{aligned}
& \partial_{i}\left(X \otimes H_{\alpha_{1}}^{\prime a_{1}} \cdots H_{\alpha_{N}}^{\prime a_{N}} \otimes \sigma^{c} \otimes Y^{\circ} \otimes H_{\alpha_{1}}^{\prime \circ b_{1}} \cdots H_{\alpha_{N}}^{\prime \circ b_{N}} \otimes \sigma^{\circ d}\right) \\
& \quad=a_{i} \cdot X \otimes H_{\alpha_{1}}^{\prime a_{1}} \cdots H_{\alpha_{i}}^{\prime a_{i}-1} \cdots H_{\alpha_{N}}^{\prime a_{N}} \otimes \sigma^{c} \otimes Y^{\circ} \otimes H_{\alpha_{1}}^{\prime o b_{1}} \cdots H_{\alpha_{N}}^{\prime \circ b_{N}} \otimes \sigma^{\circ d},
\end{aligned}
$$




$$
\begin{aligned}
\partial_{i}^{\circ}\left(X \otimes H_{\alpha_{1}}^{\prime a_{1}} \cdots H_{\alpha_{N}}^{\prime a_{N}} \otimes \sigma^{c} \otimes Y^{\circ} \otimes H_{\alpha_{1}}^{\prime \circ b_{1}} \cdots H_{\alpha_{N}}^{\prime \circ b_{N}} \otimes \sigma^{\circ d}\right) \\
\quad=b_{i} \cdot X \otimes H_{\alpha_{1}}^{\prime a_{1}} \cdots H_{\alpha_{N}}^{\prime a_{N}} \otimes \sigma^{c} \otimes Y^{\circ} \otimes H_{\alpha_{1}}^{\prime o b_{1}} \cdots H_{\alpha_{i}}^{\prime o b_{i}-1} \cdots H_{\alpha_{N}}^{\prime \circ b_{N}} \otimes \sigma^{\circ d}
\end{aligned}
$$

where the elements

$$
X \otimes H_{\alpha_{1}}^{\prime a_{1}} \cdots H_{\alpha_{N}}^{\prime a_{N}} \otimes \sigma^{c} \otimes Y^{\circ} \otimes H_{\alpha_{1}}^{\prime \circ b_{1}} \cdots H_{\alpha_{N}}^{\prime \circ b_{N}} \otimes \sigma^{\circ d}
$$

are basis elements of

$$
\mathfrak{D}^{\prime} \simeq N^{\prime} \hat{\otimes} \subseteq\left[\mathscr{H}^{R^{\prime}}\right] \hat{\otimes} R^{\prime}\langle\sigma\rangle \hat{\otimes} N^{\prime \circ} \hat{\otimes} \subseteq\left[\mathscr{H}^{R^{\prime}}\right]^{\circ} \hat{\otimes} R^{\prime}\left\langle\sigma^{\circ}\right\rangle
$$

(see (2.8.3)). Then we have:

$$
\begin{aligned}
& \partial_{i}\left(X \otimes K_{1}^{l_{1}} \cdots K_{n}^{l_{n}} \otimes \sigma^{c} \otimes Y^{\circ} \otimes K_{1}^{\circ m_{1}} \cdots K_{n}^{o m_{n}} \otimes \sigma^{\circ d}\right) \\
& =l_{i} \sqrt{h} \cdot\left(X \otimes K_{1}^{l_{1}} \cdots K_{n}^{l_{n}} \otimes \sigma^{c} \otimes Y^{\circ} \otimes K_{1}^{o m_{1}} \cdots K_{n}^{o m_{n}} \otimes \sigma^{\circ d}\right), \\
& \partial_{i}^{\circ}\left(X \otimes K_{1}^{l_{1}} \cdots K_{n}^{l_{n}} \otimes \sigma^{c} \otimes Y^{\circ} \otimes K_{1}^{\circ m_{1}} \cdots K_{n}^{\circ m_{n}} \otimes \sigma^{\circ d}\right) \\
& =m_{i} \sqrt{h} \cdot\left(X \otimes K_{1}^{l_{1}} \cdots K_{n}^{l_{n}} \otimes \sigma^{c} \otimes Y^{\circ} \otimes K_{1}^{o m_{1}} \cdots K_{n}^{\circ m_{n}} \otimes \sigma^{\circ d}\right) .
\end{aligned}
$$

Hence (2.9.3) is the eigenspace decomposition of $U$ with respect to $\partial_{i}$ and $\partial_{i}^{\circ}$.

Now we state the main theorem of $\S 2$. We put $q=e^{h}$.

Theorem 2.9.4. Let $\left(\mathscr{E}, \Pi=\left\{\alpha_{1}, \cdots, \alpha_{n}\right\}, p\right)$ be a triple system and $D=\operatorname{diag}\left(d_{1}, \cdots, d_{n}\right)\left(d_{i} \in \frac{1}{2} \mathbb{Z} \backslash\{0\}\right) . \quad$ Put $q_{i}=q^{d_{i}} \in R$. Then there exists a unique topologically free $R$-Hopf algebra $U_{h}^{\sigma}=U_{h}^{\sigma}((\mathscr{E}, \Pi, p), D)$ satisfying the following conditions:

(i) The R-algebra $U_{h}^{\sigma}$ contains $\mathfrak{S}\left[\mathscr{H}^{R}\right], R\langle\sigma\rangle, N_{+}, N_{+}^{\circ}$ as $R$-subalgebras. Here $N_{+}^{\circ}$ is another algebra isomorphic to $N_{+}$. For $Y \in N_{+}$, we denote the corresponding element of $N_{+}^{\circ}$ by $Y^{\circ}$. As topological R-modules,

$$
U_{h}^{\sigma} \simeq N_{+} \hat{\otimes} \subseteq\left[\mathscr{H}^{R}\right] \hat{\otimes} R\langle\sigma\rangle \hat{\otimes} N_{+}^{\circ}\left(X \cdot P \cdot \sigma^{c} \cdot Y^{\circ} \leftarrow X \otimes P \otimes \sigma^{c} \otimes Y^{\circ}\right) .
$$


(ii) There is a topological $R^{\prime}$-Hopf algebra homomorphism $\Omega^{\prime}: \mathfrak{D}^{\prime} \rightarrow U_{h}^{\sigma} \otimes R^{\prime}$ such that

$$
\begin{aligned}
& \Omega^{\prime}\left(E_{i}\right)=E_{i}(1 \leq i \leq n), \Omega^{\prime}\left(H^{\prime}\right)=-\Omega^{\prime}\left(H^{\circ}\right)=\sqrt{h} H(H \in \mathscr{H}) . \\
& \Omega^{\prime}(\sigma)=\Omega^{\prime}\left(\sigma^{\circ}\right)=\sigma, \Omega^{\prime}\left(E_{i}^{\circ}\right)=\left(q_{i}^{-1}-q_{i}\right) E_{i}^{\circ}(1 \leq i \leq n) .
\end{aligned}
$$

Proof. StepI. Put $E_{i}^{\circ}=F_{i} \sigma^{p\left(\alpha_{2}\right)} \in \tilde{U}_{h}^{\sigma}(1 \leq i \leq n)$. By Lemma 2.5.1, we see that there exists a topological $R^{\prime}$-Hopf algebra homomorphism $\tilde{\Omega}^{\prime}$ : $\tilde{\mathfrak{D}}^{\prime} \rightarrow \tilde{U}_{h}^{\sigma} \otimes R^{\prime}$ satisfying the conditions (2.9.5). Let $\tilde{N}_{+}^{o}$ be the unital subalgebra of $\tilde{U}_{h}^{\sigma}$ algebraically generated by the elements $E_{i}^{\circ}(1 \leq i \leq n)$. By Lemma 2.1.4, it is clear that

$$
\tilde{U}_{h}^{\sigma} \simeq \tilde{N}_{+} \hat{\otimes} \mathfrak{S}\left[\mathscr{H}^{R}\right] \hat{\otimes} R\langle\sigma\rangle \hat{\otimes} \tilde{N}_{+}^{\circ} .
$$

Step II. We construct $U_{h}^{\sigma}$ as a quotient of $\tilde{U}_{h}^{\sigma}$. Set $J_{1}=$ $\overline{I_{+} \cdot \Im\left[\mathscr{H}^{R}\right] \cdot R\langle\sigma\rangle \cdot \tilde{N}_{+}^{\circ}}$ and $J_{2}=\overline{N_{+} \cdot S\left[\mathscr{H}^{R}\right] \cdot R\langle\sigma\rangle \cdot I_{+}^{\circ}}$ where $I_{+}^{\circ}=\left\{Y^{\circ} \mid Y \in I_{+}\right\}$. We claim:

$$
J_{1} \text { and } J_{2} \text { are Hopf ideals of } \tilde{U}_{h}^{\sigma} \text {. }
$$

Assume this fact for a moment. We define the Hopf algebra $U_{h}^{\sigma}$ by $\tilde{U}_{h}^{\sigma} /\left(J_{1}+J_{2}\right)$. By Lemma 2.8.1, there exists an $R^{\prime}$-Hopf algebra homomorphism $\Omega^{\prime}: \mathfrak{D}^{\prime} \rightarrow U_{h}^{\sigma} \otimes R^{\prime}$ naturally induced from $\tilde{\Omega}^{\prime}: \tilde{\mathfrak{D}} \rightarrow \tilde{U}_{h}^{\sigma} \otimes R^{\prime}$ of Step 1 . By (2.9.6) and the definition of $U_{h}^{\sigma}$, it is clear that $U_{h}^{\sigma} \simeq N_{+} \hat{\otimes} \subseteq\left[\mathscr{H}^{R}\right] \hat{\otimes}$ $R\langle\sigma\rangle \otimes N_{+}^{\circ}$ as $R$-modules. In particular, $U_{h}^{\sigma}$ is topologically free. Since $h\left(U_{h}^{\sigma} \otimes R^{\prime}\right) \subset \operatorname{Im} \Omega^{\prime}$, the product in $U_{h}^{\sigma}$ is uniquely determined by $\Omega^{\prime}$. Hence the uniquiness of $U_{h}^{\sigma}$ follows.

It remains to prove (2.9.7). We shall prove this only for $J_{1} ; J_{2}$ can be treated similarly.

First we prove that $J_{1}$ is a two-sided ideal of the algebra $\tilde{U}_{h}^{\sigma}$. Evidently, $J_{1}$ is a right ideal and $E_{i} \cdot J_{1} \subset J_{1}(1 \leq i \leq n), H \cdot J_{1} \subset J_{1}(H \in \mathscr{H}), \sigma \cdot J_{1} \subset J_{1}$. Recall $I_{+}=\underset{\lambda \in P_{+}}{\bigoplus} I_{+, \lambda}$. By Lemma 2.9.1, $J_{1}$ is a direct summand of $\tilde{U}_{h}^{\sigma}$. Hence it is enough to show that

$$
\left(q_{i}^{-1}-q_{i}\right) E_{i}^{\circ} \cdot I_{+, \lambda} \subset I_{+, \lambda} \cdot E_{i}^{\circ}
$$




$$
+I_{+, \lambda-\alpha_{i}} \cdot \exp \left(-h H_{\alpha_{i}}\right) \sigma^{p\left(\alpha_{2}\right)}+I_{+, \lambda-\alpha_{i}} \cdot \exp \left(h H_{\alpha_{i}}\right) \sigma^{p\left(\alpha_{i}\right)}
$$

Let $X$ be an element of $I_{+, \lambda}$. By Lemma 2.5.1, we have that

$$
E_{i}^{\circ} \cdot X=X \cdot E_{i}^{\circ}+X^{(1)} \cdot \exp \left(\sqrt{h} H_{\alpha_{i}}^{\circ}\right) \sigma^{\circ p\left(\alpha_{i}\right)}+X^{(2)} \cdot \exp \left(\sqrt{h} H_{\alpha_{i}}^{\prime}\right) \sigma^{p\left(\alpha_{i}\right)}
$$

with some $X^{(1)}, X^{(2)} \in \tilde{N}_{+, \lambda-\alpha_{i}}$ in the algebra $\tilde{D}^{\prime}$. Let $\Psi: \tilde{D}^{\prime} \rightarrow \mathfrak{D}^{\prime}$ be the Hopf algebra homorphism defined in Lemma 2.8.1. By Lemma 2.9.2, $X^{(1)}$, $X^{(2)} \in \operatorname{Ker} \Psi$. Hence, by Lemma 2.8.1, $X^{(1)}, X^{(2)} \in I_{+, \lambda-\alpha_{i}}$. If we let operate $\tilde{\Omega}^{\prime}$ on the left and right hand sides of (2.9.9), we obtain:

$$
\begin{aligned}
\left(q_{i}^{-1}\right. & \left.-q_{i}\right) E_{i}^{\circ} \cdot X \\
& =\left(q_{i}^{-1}-q_{i}\right) X \cdot E_{i}^{\circ}+X^{(1)} \cdot \exp \left(-h H_{\alpha_{i}}\right) \sigma^{p\left(\alpha_{i}\right)}+X^{(2)} \cdot \exp \left(h H_{\alpha_{i}}\right) \sigma^{p\left(\alpha_{i}\right)}
\end{aligned}
$$

Hence (2.9.8) follows. Thus we showed that $J_{1}$ is a two-sided ideal.

Noting that $\operatorname{Ker} \Psi$ is a Hopf ideal, and using an argument similar to the above, we have

$$
\begin{aligned}
\Delta(X)= & \sum_{\mu, v \in P_{+}, \mu+v=\lambda}\left\{X_{\mu}^{(1)} \cdot \exp \left(h H_{v}\right) \sigma^{p(v)} \otimes Y_{v}^{(1)}\right. \\
& \left.+Y_{\mu}^{(2)} \cdot \exp \left(h H_{v}\right) \sigma^{p(v)} \otimes X_{v}^{(2)}\right\}, X \in I_{+, \lambda},
\end{aligned}
$$

with some $X_{\mu}^{(1)} \in I_{+, \mu}, \quad X_{v}^{(2)} \in I_{+, v}, \quad Y_{v}^{(1)} \in N_{+, v}, \quad Y_{\mu}^{(2)} \in N_{+, \mu} . \quad$ Hence $\Delta\left(J_{1}\right) \subset$ $J_{1} \otimes \tilde{U}_{h}^{\sigma}+\tilde{U}_{h}^{\sigma} \otimes J_{1}$. Similarly we have:

$$
S\left(I_{+, \lambda}\right) \subset I_{+, \lambda} \exp \left(h H_{\lambda}\right) \cdot \sigma^{p(\lambda)} .
$$

Hence $S\left(J_{1}\right) \subset J_{1}$. It is clear that $\varepsilon\left(J_{1}\right)=0$. Hence (2.9.7) is proved. This completes the proof.

By Proposition 1.7.1 and Lemma 2.4.1, we have:

Lemma 2.9.10. Let $\Omega^{\prime}: \mathfrak{D}^{\prime} \rightarrow U_{h}^{\sigma} \otimes R^{\prime}$ be the Hopf algebra homomorphism defined in Theorem 2.9.4. Let $K^{\prime}$ (resp. $K$ ) be the fraction field of $R^{\prime}$ (resp. $R)$. Denote the scalar extention $\Omega^{\prime} \otimes \mathrm{id}_{K^{\prime}}: \mathfrak{D}^{\prime} \otimes K^{\prime} \rightarrow U_{h}^{\sigma} \otimes K^{\prime}$ of $\Omega^{\prime}$ again by $\Omega^{\prime}$. For $\lambda \in P_{+}$, put $p_{\lambda}=\operatorname{rank} N_{+, \lambda}$, and let $\left\{e_{(\lambda, i)}\right\}_{1 \leq i \leq p_{\lambda}},\left\{e^{(\lambda, i)}\right\}_{1 \leq i \leq p_{\lambda}}$ be bases of $N_{+, \lambda} \otimes K$ such that $\left\langle e_{(\lambda, i)}, e^{(\lambda, j)}\right\rangle=\delta_{i j}$. Let $\left\{\varepsilon_{i}\right\}_{1 \leq i \leq N}$ be a basis of $\mathscr{E}$ 
such that $\left(\varepsilon_{i}, \varepsilon_{j}\right)=\delta_{i j} . \quad$ Put $t_{0}=\sum_{i=1}^{N} H_{\varepsilon_{i}} \otimes H_{\varepsilon_{i}} \in \mathscr{H} \otimes \mathscr{H} . \quad$ If the element

$$
\mathscr{R}=\left\{\sum_{\lambda \in P_{+}} \sum_{i=1}^{p \lambda} \Omega^{\prime}\left(e_{(\lambda, i)}\right) \otimes \Omega^{\prime}\left(e^{(\lambda, i)}\right)\right\} \cdot \exp \left(-h t_{0}\right) \cdot\left\{\frac{1}{2_{c, d=0}} \sum^{1}(-1)^{c d} \sigma^{c} \otimes \sigma^{d}\right\}
$$

converges in $U_{h}^{\sigma} \otimes U_{h}^{\sigma} \otimes K^{\prime}$ and $\mathscr{R} \in U_{h}^{\sigma} \hat{\otimes} U_{h}^{\sigma}$, then $\left(U_{h}^{\sigma}, \Delta, \mathscr{R}\right)$ is a quasitriangular Hopf algebra.

By Proposition 1.9.2 and Theorem 2.9.4, we can easily show:

Corollary 2.9.11. Let $F_{i}=E_{i}^{\circ} \sigma^{p\left(\alpha_{i}\right)} \in U_{h}^{\sigma}(1 \leq i \leq n) . \quad$ Let $U_{h}=U_{h}(\Pi, p)=$ $U_{h}((\mathscr{E}, \Pi, p), D)$ be an $R$-subalgebra of $U_{h}^{\sigma} h$-adically generated by the elements $E_{i}, F_{i}(1 \leq i \leq n)$ and $H \in \mathscr{H}$. Then we have

(i) $U_{h}^{\sigma}=U_{h} \oplus U_{h} \cdot \sigma$. In particular, $U_{h}$ is topologically free.

(ii) $U_{h}$ has a superalgebra structure such that the parities of $E_{i}, F_{i}$ and $H \in \mathscr{H}$ are $p\left(\alpha_{i}\right), p\left(\alpha_{i}\right)$ and 0 respectively.

(iii) $U_{h}$ has a topological Hopf superalgebra structure such that the Hopf algebra $U_{h}^{\sigma}$ is isomorphic to the Hopf algebra in Proposition 1.9.1 for $U_{h}$.

2.10. Here we show that, if $\Pi=\left\{\alpha_{1}, \cdots, \alpha_{n}\right\}$ is the set of simple roots of a symmetrizable Kac-Moody Lie algebra $\boldsymbol{G}$ and $p\left(\alpha_{i}\right)=0$ $(1 \leq i \leq n)$, then $U_{h}$ is isomorphic to the quantized enveloping algebra $U_{h}(G)$ introduced by Drinfeld [4] and Jimbo [5]. More precisely, we obtain the following theorem.

Theorem 2.10.1. Suppose $p\left(\alpha_{i}\right)=0$ for all $i$. Assume that $\left(\alpha_{i}, \alpha_{i}\right)>0$ $(1 \leq i \leq n),\left(\alpha_{i}, \alpha_{j}\right) \leq 0(i \neq j)$ and $a_{i j}=2\left(\alpha_{i}, \alpha_{j}\right) /\left(\alpha_{i}, \alpha_{i}\right) \in Z$ Z. Let $d_{i}=\frac{\left(\alpha_{i}, \alpha_{i}\right)}{2}(1 \leq i \leq n)$ and $\boldsymbol{D}=\operatorname{diag}\left(d_{1}, \cdots, d_{n}\right)$. Then $I_{+}$is the ideal of $\tilde{N}_{+}$generated by the elements

$$
\sum_{v=0}^{1-a_{i j}}(-1)^{v}\left[\begin{array}{c}
1-a_{i j} \\
v
\end{array}\right]_{q_{i}} E_{i}^{v} E_{j} E_{i}^{1-a_{i j}-v} \quad(i \neq j)
$$


where $q_{i}=\exp \left(h d_{i}\right)$ and

$$
\left[\begin{array}{c}
1-a_{i j} \\
v
\end{array}\right]_{q_{i}}=\prod_{s=1}^{v} \frac{q_{i}^{2-a_{i j}-s}-q_{i}^{a_{i j}+s-2}}{q_{i}^{s}-q_{i}^{-s}} \in R .
$$

Proof. Let us denote $y_{i j} \in \tilde{N}_{+}$the element (2.10.2). By a direct computation, we see that $\Delta^{\prime}\left(y_{i j}\right)=y_{i j} \otimes 1+\exp \left(\sqrt{h} H_{\left(\left(1-a_{i j}\right) \alpha_{i}+\alpha_{j}\right)}^{\prime}\right) \otimes y_{i j}$. Hence the ideal of $\tilde{U}_{\sqrt{h}}^{\prime} b_{+}^{\sigma}$ generated by the elements $y_{i j}$ is the bi-ideal. Hence, by Lemma 2.6.1, $y_{i j} \in I_{+}$. Put $\mathfrak{U}=U_{h} / h U_{h}$ where $U_{h}$ is of Corollary 2.9.11. Since $U(G)$ is defined by the Serre relations, there exists a natural epimorphism $\psi: U(G) \rightarrow \mathfrak{U}$.

Let $U(\boldsymbol{G})=U\left(\boldsymbol{n}_{+}\right) \otimes U(\mathscr{H}) \otimes U\left(\boldsymbol{n}_{-}\right)$be the triangular dcomposition. Put $\mathfrak{N}_{+}=N_{+} / h N_{+}$. Let $U\left(\boldsymbol{n}_{+}\right)_{\gamma}$ and $\mathfrak{N}_{+, \gamma}$ denote the weight space of a weight $\gamma \in P_{+}$. Let $\mathscr{V}(\lambda)$ and $\mathfrak{B}(\lambda)$ denote the irreducible highest weight module with highest weight $\lambda$ of $U(G)$ and $\mathcal{U}$ respectively. Let $\mathscr{V}(\lambda)_{\lambda-\gamma} \subset \mathscr{V}(\lambda)$ and $\mathfrak{B}(\lambda)_{\lambda-\gamma} \subset \mathfrak{B}(\lambda)$ be the weight spaces of weight $\lambda-\gamma$. From a well-known fact in the representation theory of $G$ (see the formula (10.4.6) in [6]), we can see that, if $\lambda$ is sufficiently large as compared with $\gamma \in P_{+}, \operatorname{dim} U\left(\boldsymbol{n}_{+}\right)_{\gamma}=$ $\operatorname{dim} \mathscr{V}(\lambda)_{\lambda-\gamma}$. On the other hand, using $\psi: U(G) \rightarrow \mathfrak{U}$, we can regard $\mathfrak{B}(\lambda)$ as an irreducible $U(G)$-module isomorphic to $\mathscr{V}(\lambda)$. Hence we have:

$$
\operatorname{dim} U\left(\boldsymbol{n}_{+}\right)_{\gamma}=\operatorname{dim} \mathscr{V}(\lambda)_{\lambda-\gamma}=\operatorname{dim} \mathfrak{B}(\lambda)_{\lambda-\gamma} \leq \operatorname{dim} \mathfrak{N}_{+, \gamma} .
$$

Since $\psi$ is an epimorphism, $\operatorname{dim} U\left(\boldsymbol{n}_{+}\right)_{\gamma} \geq \operatorname{dim} \mathfrak{N}_{+, \gamma} . \quad$ Hence we have

$$
\operatorname{dim} U\left(\boldsymbol{n}_{+}\right)_{\gamma}=\operatorname{dim} \mathfrak{N}_{+, \gamma} .
$$

Note that $\tilde{N}_{+, \lambda}$ is a free $R$-module of finite rank and $I_{+, \lambda}$ is a direct summand of $\tilde{N}_{+, \lambda}$ (see Lemma 2.9.1). Hence, if $w_{u}\left(1 \leq u \leq \operatorname{rank} I_{+, \lambda}\right)$ are elements of $I_{+, \lambda}$ such that $\left\{w_{u}+h I_{+, \lambda}\right\}$ is a $C$-basis of $I_{+, \lambda} / h I_{+, \lambda}$, then $\left\{w_{u}\right\}$ is an $R$-basis of $I_{+, \lambda}$. In particular, by (2.10.3), we can put

$$
w_{u}=E_{u_{1}} \cdots E_{u_{k-1}} y_{u_{k}, u_{k+1}} E_{u_{k+2}} \cdots E_{u_{p}} \in I_{+, \lambda}\left(\lambda=-a_{u_{k}, u_{k+1}} \alpha_{u_{k}}+\sum_{t=1}^{p} \alpha_{u_{t}}\right) .
$$


Hence the theorem follows.

Remark 2.10.4. We remark that the above theorem can also be proved by using Tanisaki's result; as an immediately consequence of Proposition 2.4.1 in [20], we can show the non-degeneracy of the Hopf pairing $\langle$,$\rangle :$ $U_{\boldsymbol{h}}\left(\boldsymbol{n}_{+}\right) \times U_{\boldsymbol{h}}\left(\boldsymbol{n}_{+}\right) \rightarrow R$ where $U_{\boldsymbol{h}}\left(\boldsymbol{n}_{+}\right)=\tilde{N}_{+} /\left(y_{i j}(1 \leq i \neq j \leq n)\right)$. Hence $I_{+}=\left(y_{i j}\right)$.

\section{$\S 3$. Root Systems of Simple Lie Superalgebras}

3.1. Let $\mathscr{G}=\mathscr{G}_{0} \oplus \mathscr{G}_{1}$ be a finite dimensional complex simple Lie superalgebra of type $A-G$. Let $\left(\Phi, \mathscr{E}_{R}\right)$ be a root system of $\mathscr{G}$. For terminologies related to simple Lie superalgebras, see [7]. Here $\mathscr{E}_{\boldsymbol{R}}$ is an $N$-dimensional real vector space with a non-degenerate symmetric bilinear form ( , ): $\mathscr{E}_{\boldsymbol{R}} \times \mathscr{E}_{\boldsymbol{R}} \rightarrow \boldsymbol{R}$ and $\Phi\left(\subset \mathscr{E}_{\boldsymbol{R}}\right)$ is the set of roots. Put $\mathscr{E}=\boldsymbol{C} \otimes \mathscr{E}_{\boldsymbol{R}}$. Let $\Pi=\left\{\alpha_{1}, \cdots, \alpha_{n}\right\}$ be a set of simple roots and $p: \Pi \rightarrow\{0,1\}=\boldsymbol{Z} / 2 \boldsymbol{Z}$ the parity function. Put $P=\boldsymbol{Z} \alpha_{1}+\cdots+\boldsymbol{Z} \alpha_{n}(\subset \mathscr{E})$. We extend $p$ to the function $p$ : $P \rightarrow \boldsymbol{Z} / 2 \boldsymbol{Z}$ additively. We assume that the triple $(\mathscr{E}, \Pi, p)$ is of distinguished type if $\mathscr{G}$ is of type $F_{4}$ or $G_{3}$. We do not treat $(\mathscr{E}, \Pi, p)$ of type $D(2,1 ; \alpha)$. That case is easy. However we need some unpleasant notation for type $D(2,1 ; \alpha)$. To fix notation, we list below Dynkin diagrams, systems of simple roots $\Pi=\left\{\alpha_{1}, \cdots, \alpha_{n}\right\}$, the set of positive roots $\Phi_{+}$and parity function $p$ : $\Pi \rightarrow\{0,1\}$ of triples $(\mathscr{E}, \Pi, p)$ of type $A-G$. We put $N=n+1$ if $(\Phi, \Pi, p)$ is of type $A$, and $N=n$ otherwise. Let $\left\{\bar{\varepsilon}_{i}(1 \leq i \leq N)\right\}$ be a fixed orthogonal basis of $\mathscr{E}_{R}$; the values of $\left(\bar{\varepsilon}_{i}, \bar{\varepsilon}_{i}\right)$ are given below. The element of $\mathscr{E}_{R}$ written under the dot with the $i$-th label is the simple root $\alpha_{i}$. Note that the numbering of $\alpha_{i}$ 's is not the standard one for types $F_{4}$ and $G_{3}$. In the following diagram, the parity function $p: \Pi \rightarrow\{0,1\}$ is defined as follows. The $\operatorname{dot} \times$ at the $i$-th label stands for the $\operatorname{dot} \bigcirc$ (resp. $\otimes)$ if $\left(\alpha_{i}, \alpha_{i}\right) \neq 0$ (resp. $\left(\alpha_{i}, \alpha_{i}\right)=0$ ). If the $i$-th dot is $\bigcirc, \otimes$ or, then we define $p\left(\alpha_{i}\right)=0,1,1$ respectively. We also give the diagonal matrix $\boldsymbol{D}=\operatorname{diag}\left(d_{1}, \cdots, d_{n}\right)$ such that $A=D^{-1}\left[\left(\alpha_{i}, \alpha_{j}\right)\right]$ is the Cartan matrix of $(\Phi, \Pi)$.

(i) Types $A, B, C$ or $D$. For types $A-D$, we put $\left(\bar{\varepsilon}_{i}, \bar{\varepsilon}_{j}\right)= \pm \delta_{i j}$ $(1 \leq i, j \leq N)$, where we can arbitrarily choose the signs of $\left(\bar{\varepsilon}_{i}, \bar{\varepsilon}_{i}\right)$. 


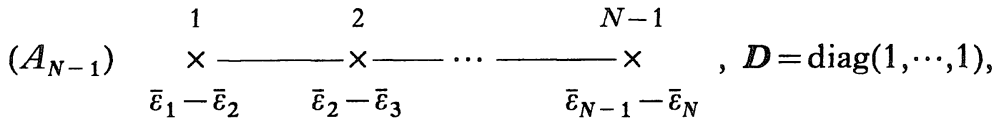

$$
\begin{aligned}
& \Phi_{+}=\left\{\bar{\varepsilon}_{i}-\bar{\varepsilon}_{j}(1 \leq i<j \leq N)\right\} \text {. }
\end{aligned}
$$
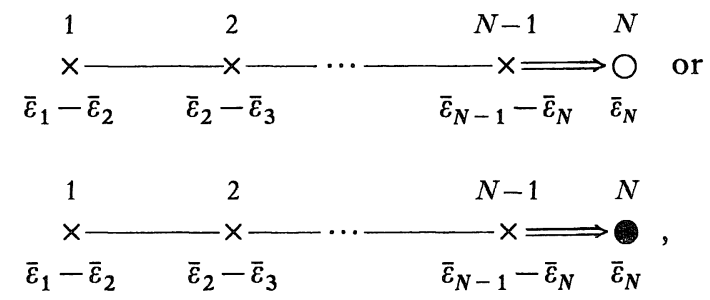

$\left(C_{N}\right)$

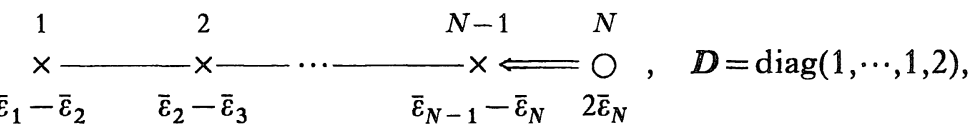

$$
\begin{aligned}
& \Phi_{+}=\left\{\bar{\varepsilon}_{i} \pm \bar{\varepsilon}_{j}(1 \leq i<j \leq N), 2 \bar{\varepsilon}_{i}\left(1 \leq i \leq N, p\left(\bar{\varepsilon}_{i}-\bar{\varepsilon}_{N}\right)=0\right)\right\} .
\end{aligned}
$$
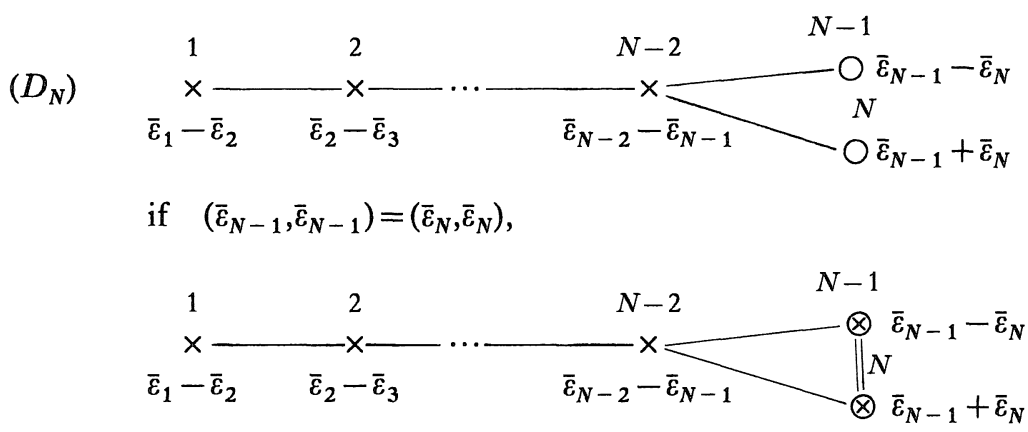

if $\left(\bar{\varepsilon}_{N-1}, \bar{\varepsilon}_{N-1}\right)=-\left(\bar{\varepsilon}_{N}, \bar{\varepsilon}_{N}\right)$,

$\boldsymbol{D}=\operatorname{diag}(1, \cdots, 1)$,

$\Phi_{+}=\left\{\bar{\varepsilon}_{i} \pm \bar{\varepsilon}_{j}(1 \leq i<j \leq N), 2 \bar{\varepsilon}_{i}\left(1 \leq i \leq N, p\left(\bar{\varepsilon}_{i}-\bar{\varepsilon}_{N}\right)=1\right)\right\}$.

(ii) Types $F_{4}$ and $G_{3}$. 


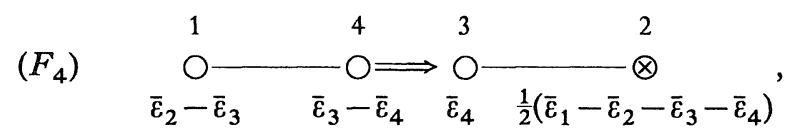

$\left[\left(\bar{\varepsilon}_{i}, \bar{\varepsilon}_{j}\right)\right\}=\operatorname{diag}(6,-2,-2,-2), \boldsymbol{D}=\operatorname{diag}(2,1,1,2)$,

$\Phi_{+}=\left\{n_{1} \alpha_{1}+n_{4} \alpha_{4}+n_{3} \alpha_{3}+n_{2} \alpha_{2} \mid\left(n_{1}, n_{4}, n_{3}, n_{2}\right)=(1,0,0,0), \quad(1,1,0,0), \quad(1,1,1,0)\right.$, $(1,1,2,0),(1,1,1,1),(1,2,2,0),(1,1,2,1),(1,2,2,1),(1,2,3,1),(1,2,3,2),(0,0,0,1)$, $(0,0,1,1),(0,1,1,1),(0,1,2,1),(0,0,1,0),(0,1,2,0),(0,1,1,0),(0,1,0,0)\}$.

$\left(G_{3}\right)$

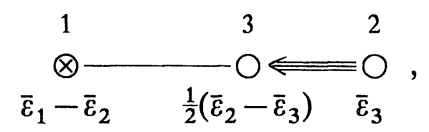

$\left[\left(\bar{\varepsilon}_{i}, \bar{\varepsilon}_{j}\right)\right]=\operatorname{diag}(-2,2,6), \boldsymbol{D}=\operatorname{diag}(1,3,1)$, $\Phi_{+}=\left\{n_{1} \alpha_{1}+n_{3} \alpha_{3}+n_{2} \alpha_{2} \mid\left(n_{1}, n_{3}, n_{2}\right)=(1,0,0),(1,1,0),(1,1,1),(1,2,1),(1,3,1)\right.$, $(1,3,2),(1,4,2),(2,4,2),(0,0,1),(0,1,1),(0,3,2)(0,2,1),(0,3,1),(0,1,0)\}$.

3.2. Let $\Phi_{+}$be the set of positive roots with respect to $\Pi$. Put $\Phi_{+}^{\text {red }}=\left\{\beta \in \Phi_{+} \mid \frac{1}{2} \beta \notin \Phi_{+}\right\}$. We define the partial order $<$on $\Phi_{+}^{\text {red }}$ as follows. Given $\beta=c_{1} \alpha_{1}+\cdots+c_{n} \alpha_{n} \in \Phi_{+}^{\text {red }}$, we define integers $h t(\beta), g(\beta), c_{\beta} \in Z_{+}$ by $h t(\beta)=c_{1}+\cdots+c_{n}, g(\beta)=\min \left\{i \mid c_{i} \neq 0\right\}, c_{\beta}=c_{g(\beta)}$ respectively. Define a half integer $h t^{\prime}(\beta)$ by $h t^{\prime}(\beta)=h t(\beta) / c_{\beta}$. Let $\alpha, \beta$ be elements of $\Phi_{+}^{\text {red }}$. We say that $\alpha<\beta$ if they satisfy one of the following

(i) $g(\alpha)<g(\beta)$,

(ii) $g(\alpha)=g(\beta)$ and $h t^{\prime}(\alpha)<h t^{\prime}(\beta)$

or

(iii) $\Phi_{+}^{\text {red }}$ is of type $D_{N}, p\left(\bar{\varepsilon}_{i}-\bar{\varepsilon}_{N}\right)=1$ and $\alpha=\bar{\varepsilon}_{i}-\bar{\varepsilon}_{N}, \beta=2 \bar{\varepsilon}_{i}$ or $\alpha=2 \bar{\varepsilon}_{i}$, $\beta=\bar{\varepsilon}_{i}+\bar{\varepsilon}_{N}$, or, $\alpha=\bar{\varepsilon}_{i}-\bar{\varepsilon}_{N}, \beta=\bar{\varepsilon}_{i}+\bar{\varepsilon}_{N}$. 
For, $\alpha, \beta \in \Phi_{+}^{\text {red }}$, let $\Phi_{+}^{\text {red }}(<\alpha)=\left\{\gamma \in \Phi_{+}^{\text {red }} \mid \gamma<\alpha\right\}, \quad \Phi_{+}^{\text {red }}(\alpha<\beta)=\left\{\gamma \in \Phi_{+}^{\text {red }} \mid \alpha<\right.$ $\gamma<\beta\}, \Phi_{+}^{\text {red }}(\beta<)=\left\{\gamma \in \Phi_{+}^{\text {red }} \mid \beta<\gamma\right\}$.

Let $\Phi_{+, i}^{\mathrm{red}}=\left\{\beta \in \Phi_{+}^{\mathrm{red}} \mid g(\beta)=i\right\}$. For $\alpha, \beta \in \Phi_{+, i}^{\mathrm{red}}$ let $\Phi_{+, i}^{\mathrm{red}}(<\alpha)=\Phi_{+, i}^{\mathrm{red}} \cap \Phi_{+}^{\mathrm{red}}$ $(<\alpha), \Phi_{+, i}^{\mathrm{red}}(\alpha<\beta)=\Phi_{+, i}^{\mathrm{red}} \cap \Phi_{+}^{\mathrm{red}}(\alpha<\beta), \Phi_{+, i}^{\mathrm{red}}(\beta<)=\Phi_{+, i}^{\mathrm{red}} \cap \Phi_{+}^{\mathrm{red}}(\beta<)$.

\section{§4. Defining Relations of $N_{+}$}

4.1. Keep the notation in $\S 2-3$. Assume that the triple system $\left(\mathscr{E}, \Pi=\left\{\alpha_{1}, \cdots, \alpha_{n}\right\}, p\right)$ satisfies the assumption in 3.1. Let $A=\left(a_{i j}\right)_{1 \leq i, j \leq n}=$ $D^{-1}\left(\left(\alpha_{i}, \alpha_{j}\right)\right)$ be the corresponding Cartan matrix. Let $\tilde{U}_{\sqrt{h}}^{\prime} b_{+}^{\sigma}=\tilde{N}_{+}^{\prime} \hat{\otimes}$ $\circlearrowleft\left[\mathscr{H}^{R^{\prime}}\right] \otimes R^{\prime}\langle\sigma\rangle$ be the topologically free $R^{\prime}$-Hopf algebra defined in 2.2 for the triple $(\mathscr{E}, \Pi, p)$. The purpose of this section is to define an ideal $\mathscr{I}_{+}$ (@ $I_{+}$) of $\tilde{N}_{+}$with an explicit set of generators. In $\S 10$, we will show $\mathscr{I}_{+}=I_{+}$.

4.2. We define the $\boldsymbol{Z}_{2}$-graded algebra structure on $\tilde{N}_{+}$such that the parity of $E_{i}$ is $p\left(\alpha_{i}\right)$. Denote the parity of $X \in \tilde{N}_{+}$by $p(X)$. Put $[X, Y]_{v}=X Y-(-1)^{p(X) p(Y)} v Y X$ and $[X, Y]=[X, Y]_{1}$ where $p(X)$ and $p(Y)$ are the parities of $X$ and $Y$. Set $\left[\begin{array}{c}m+n \\ n\end{array}\right]_{t}=\prod_{i=0}^{n-1}\left(\left(t^{m+n-i}-t^{-m-n+i}\right) /\left(t^{i+1}-t^{-i-1}\right)\right)$ $\in C[t]$. Put $q=e^{h}, v_{i}=q^{\left(\bar{\varepsilon}_{i}, \bar{\varepsilon}_{i}\right)}$ and $q_{i}=q^{d_{i}}$.

Definition 4.2.1. Let $\mathscr{I}_{+}$be the ideal of $\tilde{N}_{+}$generated by the following elements:

(i) $\left[E_{i}, E_{j}\right]$ for $1 \leq i, j \leq n$ such that $a_{i j}=0$,

(ii) $\sum_{v=0}^{1+\left|a_{i j}\right|}(-1)^{v}\left[\begin{array}{c}1+\left|a_{i j}\right| \\ v\end{array}\right]_{q_{i}} E_{i}^{1+\left|a_{i j}\right|-v} E_{j} E_{i}^{v}$ for $1 \leq i \neq j \leq n$

such that $p\left(\alpha_{i}\right)=0$,

(iii) $\left[\left[\left[E_{i}, E_{j}\right]_{v_{j}}, E_{k}\right]_{v_{j+1}}, E_{j}\right]$ with $\stackrel{i}{\times} \underset{-}{\otimes} \longrightarrow \stackrel{k}{\times}(1<j<k)$,

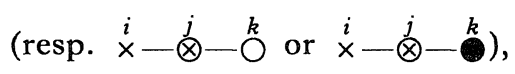

(iv) $\left[\left[\left[E_{N-1}, E_{N}\right]_{v_{N}}, E_{N}\right], E_{N}\right]_{v_{\bar{N}}^{-1}}$ with $\times \Rightarrow$ 
if $A$ is of type $B_{N}$,

(v) $\left[\left[E_{N-2}, E_{N-1}\right]_{v_{N-1}}, E_{N}\right]_{v_{N}}-\left[\left[E_{N-2}, E_{N}\right]_{v_{N-1}}, E_{N-1}\right]_{v_{N}}$

$$
N-1
$$

with

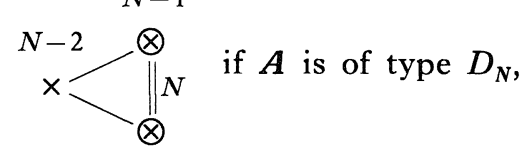

(vi) $\left[\left[\left[\left[E_{N-2}, E_{N-1}\right]_{v_{N-1}}, E_{N}\right]_{2 v_{N}},\left[E_{N-2}, E_{N-1}\right]_{v_{N-1}}\right], E_{N-1}\right]$

(resp. $\left[\left[\left[\left[\left[\left[E_{N-3}, E_{N-2}\right]_{v_{N-2}}, E_{N-1}\right]_{v_{N-1}}, E_{N}\right]_{2 v_{N}}, E_{N-1}\right]_{v_{N}}, E_{N-2}\right]_{v_{N-1}}, E_{N-1}\right]$

for

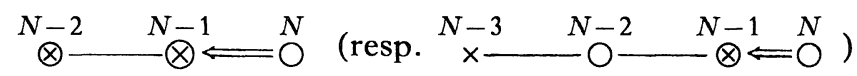

if $A$ is of type $C_{N}$.

4.3. The following proposition will be used in proving $\mathscr{I}_{+}=I_{+}$.

Proposition 4.3.1. (i) The $R^{\prime}$-submodule $\mathscr{I}_{b_{+}}^{\prime}=\left(\mathscr{I}_{+} \otimes R^{\prime}\right) \cdot \Im\left[\mathscr{H}^{R^{\prime}}\right] \cdot R^{\prime}\langle\sigma\rangle$ is a bi-ideal of $\widetilde{U}_{\sqrt{h}}^{\prime} b_{+}^{\sigma}$.

(ii) $\mathscr{I}_{+} \subset I_{+}$.

Proof. (i) Here we consider the case of the triple $(\mathscr{E}, \Pi, p)$ given by 12

$\mathrm{O}-\mathrm{O}$. In this case, the ideal $\mathscr{I}_{+}$is generated $\bar{\varepsilon}_{1}-\bar{\varepsilon}_{2} \quad \bar{\varepsilon}_{2}-\bar{\varepsilon}_{3} \quad \bar{\varepsilon}_{3}-\bar{\varepsilon}_{4}$

by the elements

$$
\begin{aligned}
& s_{\alpha_{1}+\alpha_{3}}=\left[E_{1}, E_{3}\right], s_{2 \alpha_{2}}=E_{2}^{2}, \\
& s_{2 \alpha_{1}+\alpha_{2}}=E_{1}^{2} E_{2}-\left(q+q^{-1}\right) E_{1} E_{2} E_{1}+E_{2} E_{1}^{2}, \\
& s_{2 \alpha_{2}+2 \alpha_{3}}=E_{2} E_{3}^{2}-\left(q+q^{-1}\right) E_{3} E_{2} E_{3}+E_{3}^{2} E_{2}
\end{aligned}
$$

and

$$
t_{1223}=\left[w_{123}, E_{2}\right]
$$

where $w_{123}=\left[\left[E_{1}, E_{2}\right]_{v_{2}}, E_{3}\right]_{v_{3}}$.

By an easy computation, it follows 


$$
\Delta^{\prime}\left(s_{v}\right)=s_{v} \otimes 1+\exp \left(\sqrt{h} H_{v}^{\prime}\right) \cdot \sigma^{p(v)} \otimes s_{v}
$$

for any $s_{v}$. We also have:

$$
\begin{aligned}
\Delta^{\prime}\left(w_{123}\right)= & w_{123} \otimes 1 \\
& +\left(v_{2}^{-1}-v_{2}\right)\left[E_{2}, E_{3}\right]_{v^{3}} \cdot \exp \left(\sqrt{h} H_{\bar{\varepsilon}_{1}-\bar{\varepsilon}_{2}}^{\prime}\right) \otimes E_{1} \\
& +\left(v_{3}^{-1}-v_{3}\right) E_{3} \cdot \exp \left(\sqrt{h} H_{\bar{\varepsilon}_{1}-\bar{\varepsilon}_{3}}^{\prime}\right) \cdot \sigma \otimes\left[E_{1}, E_{2}\right]_{v_{2}} \\
& +\exp \left(\sqrt{h} H_{\bar{\varepsilon}_{1}-\bar{\varepsilon}_{4}}^{\prime}\right) \cdot \sigma \otimes w_{123} .
\end{aligned}
$$

Let $\mathscr{S}$ be the ideal generated by the element $s_{2 \alpha_{2}}$. Then $\mathscr{S}$ is a bi-ideal and we have:

$$
\left.\left[\left[E_{1}, E_{2}\right]_{v_{2}}, E_{2}\right]_{v_{2}} \equiv\left[E_{2},\left[E_{2}, E_{3}\right]_{v_{3}}\right]_{v_{3}} \equiv 0 \text { (modulo } \mathscr{S}\right) \text {. }
$$

Therefore we have:

$$
\begin{aligned}
\Delta^{\prime}\left(t_{1223}\right)= & \Delta^{\prime}\left(\left[w_{123}, E_{2}\right]\right) \\
= & {\left[\Delta^{\prime}\left(w_{123}\right), E_{2} \otimes 1+\exp \left(\sqrt{h} H_{\bar{\varepsilon}_{2}-\bar{\varepsilon}_{3}}^{\prime}\right) \cdot \sigma \otimes E_{2}\right] } \\
\equiv & \left\{t_{1223} \otimes 1+0\right\} \\
& +\left\{0+\left(v_{2}^{-1}-v_{2}\right)\left[E_{2}, E_{3}\right]_{v_{3}} \cdot \exp \left(\sqrt{h} H_{\bar{\varepsilon}_{1}-\bar{\varepsilon}_{3}}^{\prime}\right) \cdot \sigma \otimes\left[E_{1}, E_{2}\right]_{v_{2}}\right\} \\
& +\left\{\left(v_{3}^{-1}-v_{3}\right)\left[E_{2}, E_{3}\right]_{v_{3}} \cdot \exp \left(\sqrt{h} H_{\bar{\varepsilon}_{1}-\bar{\varepsilon}_{3}}^{\prime}\right) \cdot \sigma \otimes\left[E_{1}, E_{2}\right]_{v_{2}}+0\right\} \\
& +\left\{0+\exp \left(\sqrt{h} H_{\bar{\varepsilon}_{1}-\bar{\varepsilon}_{4}}^{\prime}\right) \cdot \sigma \otimes t_{1223}\right\}\left(\operatorname{modulo} \mathscr{S} \otimes \widetilde{U}_{\sqrt{h}^{\prime}}^{\prime} b_{+}^{\sigma}\right. \\
& \left.+\widetilde{U}_{\sqrt{h}}^{\prime} b_{+}^{\sigma} \otimes \mathscr{S}\right) \\
= & t_{1223} \otimes 1+\exp \left(\sqrt{h} H_{\bar{\varepsilon}_{1}-\bar{\varepsilon}_{4}}^{\prime}\right) \cdot \sigma \otimes t_{1223} .
\end{aligned}
$$

Hence $\Delta^{\prime}\left(\mathscr{I}_{+}\right) \subset \mathscr{I}_{+} \otimes \tilde{U}_{\sqrt{h}}^{\prime} b_{+}^{\sigma}+\tilde{U}_{\sqrt{h}}^{\prime} b_{+}^{\sigma} \otimes \mathscr{I}_{+} \cdot$ This implies that $\mathscr{I}_{b_{+}}^{\prime}$ is a bi-ideal of $\tilde{U}_{\sqrt{h}}^{\prime} b_{+}^{\sigma}$. The other cases can be proved similarly.

(ii) It is immediate consequence of (i) and Lemma 2.6.1.

Denote the $h$-adic topological $R^{\prime}$-bi-algebra $\widetilde{U}_{\sqrt{h}}^{\prime} b_{+}^{\sigma} / \mathscr{I}_{b_{+}}^{\prime}$ by $\mathscr{U}_{\sqrt{h}}^{\prime} b_{+}^{\sigma}$. Put 
$\mathscr{N}_{+}=\tilde{N}_{+} / \mathscr{I}_{+}$and $\mathscr{N}_{+}^{\prime}=\mathscr{N}_{+} \otimes R^{\prime}\left(=\tilde{N}_{+}^{\prime} / \mathscr{I}_{+}^{\prime}\right)$. Then we have:

$$
\mathscr{U}_{\sqrt{h}}^{\prime} b_{+}^{\sigma} \simeq \mathscr{N}_{+}^{\prime} \hat{\otimes} \subseteq\left[\mathscr{H}^{R^{\prime}}\right] \hat{\otimes} R^{\prime}\langle\sigma\rangle
$$

4.4. Put $\mathscr{N}_{+, v}=\tilde{N}_{+, v} /\left(\mathscr{I}_{+} \cap \tilde{N}_{+, v}\right)\left(\subset \mathscr{N}_{+}\right)$for $v \in P_{+}$. For $v, \mu \in \mathscr{P}_{+}$and $X_{v} \in \mathscr{N}_{+, v}, X_{\mu} \in \mathscr{N}_{+, \mu}$, we put

$$
\llbracket X_{v}, X_{\mu} \rrbracket=\left[X_{v}, X_{\mu}\right]_{q^{-(v, \mu)}}
$$

Let $X_{v} \in \mathscr{N}_{+, v}, \quad X_{\mu} \in \mathscr{N}_{+, \mu}, \quad X_{\eta} \in \mathscr{N}_{+, \eta}\left(v, \mu, \eta \in P_{+}\right)$. In $\S 6-\S 9$, we shall frequently use the following identities:

$$
\begin{aligned}
& \llbracket X_{v} \cdot X_{\mu}, X_{\eta} \rrbracket=X_{v} \cdot \llbracket X_{\mu}, X_{\eta} \rrbracket+(-1)^{p(\mu) p(\eta)} q^{-(\mu, \eta)} \llbracket X_{v}, X_{\eta} \rrbracket \cdot X_{\mu}, \\
& \llbracket X_{v}, X_{\mu} \cdot X_{\eta} \rrbracket=\llbracket X_{v}, X_{\mu} \rrbracket \cdot X_{\eta}+(-1)^{p(v) p(\mu)} q^{-(v, \mu)} X_{v} \llbracket \llbracket X_{\mu}, X_{\eta} \rrbracket
\end{aligned}
$$

$$
\begin{aligned}
& \llbracket \llbracket X_{v}, X_{\mu} \rrbracket, X_{\eta} \rrbracket-\llbracket X_{v}, \llbracket X_{\mu}, X_{\eta} \rrbracket \rrbracket \\
& =(-1)^{p(\mu) p(\eta)} q^{-(\mu, \eta)} \llbracket X_{v}, X_{\eta} \rrbracket \cdot X_{\mu}-(-1)^{p(v) p(\mu)} q^{-(v, \mu)} X_{\mu} \cdot \llbracket X_{v}, X_{\eta} \rrbracket
\end{aligned}
$$

(4.4.3) If $X_{\mu}^{2}=0$,, we have:

$$
\begin{aligned}
& \llbracket \llbracket X_{v}, X_{\mu} \rrbracket, X_{\mu} \rrbracket=0, \\
& \llbracket X_{\mu}, \llbracket X_{\mu}, X_{v} \rrbracket \rrbracket=0 .
\end{aligned}
$$

(4.4.4) $\quad \llbracket \llbracket \llbracket X_{v}, X_{\mu} \rrbracket, X_{\eta} \rrbracket, X_{\chi} \rrbracket$

$$
\begin{aligned}
= & \llbracket X_{v}, \llbracket \llbracket X_{\mu}, X_{\eta} \rrbracket, X_{\chi} \rrbracket \rrbracket \\
& +(-1)^{p(\mu+\eta) p(\chi)} q^{-(\mu+\eta, \chi)} \llbracket X_{v}, X_{\chi} \rrbracket \llbracket X_{\mu}, X_{\eta} \rrbracket \\
& -(-1)^{p(v) p(\mu+\eta)} q^{-(v, \mu+\eta)} \llbracket X_{\mu}, X_{\eta} \rrbracket \llbracket X_{v}, X_{\chi} \rrbracket \\
& +(-1)^{p(\mu) p(\eta)} q^{-(\mu, \eta)} \llbracket X_{v}, X_{\eta} \rrbracket \llbracket X_{\mu}, X_{\chi} \rrbracket \\
& +(-1)^{p(\mu) p(\eta+\chi)} q^{-(\mu, \eta+\chi)} \llbracket \llbracket X_{v}, X_{\eta} \rrbracket, X_{\chi} \rrbracket X_{\mu}
\end{aligned}
$$




$$
\begin{aligned}
& -(-1)^{p(v) p(\mu)} q^{-(v, \mu)} X_{\mu} \llbracket \llbracket X_{v}, X_{\eta} \rrbracket, X_{\chi} \rrbracket \\
& -(-1)^{p(v) p(\mu)} q^{-(v, \mu)}(-1)^{p(v+\eta) p(\chi)} q^{-(v+\eta, \chi)} \llbracket X_{\mu}, X_{\chi} \rrbracket \llbracket X_{v}, X_{\eta} \rrbracket .
\end{aligned}
$$

In particular, if $\chi=\eta$, we have:

$$
\begin{aligned}
\mathbb{U} & \llbracket \llbracket X_{v}, X_{\mu} \rrbracket, X_{\eta} \rrbracket X_{\eta} \rrbracket \\
= & \llbracket X_{v}, \llbracket \llbracket \llbracket X_{\mu}, X_{\eta} \rrbracket, X_{\eta} \rrbracket \rrbracket \\
& +\left(1+(-1)^{p(\eta)} q^{-(\eta, \eta)}\right)\left\{(-1)^{p(\mu) p(\eta)} q^{-(\mu, \eta)} \llbracket X_{v}, X_{\eta} \rrbracket \llbracket X_{\mu}, X_{\eta} \rrbracket\right. \\
& \left.-(-1)^{p(v) p(\mu+\eta)} q^{-(v, \mu+\eta)} \llbracket X_{\mu}, X_{\eta} \rrbracket \llbracket \llbracket X_{v}, X_{\eta} \rrbracket\right\} \\
& +q^{-(\mu, 2 \eta)} \llbracket \llbracket X_{v}, X_{\eta} \rrbracket, X_{\eta} \rrbracket X_{\mu} \\
& -(-1)^{p(v) p(\mu)} q^{-(v, \mu)} X_{\mu} \llbracket \llbracket X_{v}, X_{\eta} \rrbracket, X_{\eta} \rrbracket .
\end{aligned}
$$

\section{§5. Root Vectors of $\mathscr{N}_{+}$}

5.1. Here we define $q$-root vectors $E_{\alpha}\left(\alpha \in \Phi_{+}^{\text {red }}\right)$ of $\mathscr{N}_{+}$.

Definition 5.1.1. For $\beta \in \Phi_{+}^{\text {red }}$, we define the element $E_{\beta} \in \mathcal{N}_{+}$ as follows. (For type $F_{4}$ (resp. $G_{3}$ ), we write $E_{a b c d}$ and $E_{a b c d}^{\prime}$ (resp. $E_{a b c}$ and $E_{a b c}^{\prime}$ ) for $E_{a \alpha_{1}+b \alpha_{4}+c \alpha_{3}+d \alpha_{2}}$ and $E_{a \alpha_{1}+b \alpha_{4}+c \alpha_{3}+d \alpha_{2}}^{\prime}$ (resp. $E_{a \alpha_{1}+b \alpha_{3}+c \alpha_{2}}$ and $\left.E_{a \alpha_{1}+b \alpha_{3}+c \alpha_{2}}^{\prime}\right)$.

(i) We put $E_{\alpha_{i}}=E_{i}(1 \leq i \leq n)$

(ii) Let $\alpha \in \Phi_{+}^{\text {red }}$ and $1 \leq i \leq n$ be such that $g(\alpha)<i$ (see 3.2 for the definition of $g(\alpha))$ and $\alpha+\alpha_{i} \in \Phi$. We put $E_{\alpha+\alpha_{i}}^{\prime}=\left[E_{\alpha}, E_{\alpha_{i}}\right]_{q}-\left(\alpha, \alpha_{i}\right)$. If $A$ is of type $B_{N}, i=N$ and $\alpha=\bar{\varepsilon}_{j}(1 \leq j \leq N-1)$, let $E_{\alpha+\alpha_{N}}=\left(q^{1 / 2}+q^{-1 / 2}\right)^{-1} E_{\alpha+\alpha_{N}}^{\prime}$. If $A$ is of type $D_{N}, i=N$ and $\alpha=\alpha_{N-1}$, let $E_{\alpha+\alpha_{N}}=\left(q+q^{-1}\right)^{-1} E_{\alpha+\alpha_{N}}^{\prime}$. If $A$ is of type $F_{4}$, let $E_{1120}=\left(q+q^{-1}\right)^{-1} E_{1120}^{\prime}$ and $E_{1232}=\left(q^{2}+1+q^{-2}\right)^{-1} E_{1232}^{\prime}$. If $A$ is of type $G_{3}$, let $E_{121}=\left(q+q^{-1}\right)^{-1} E_{121}^{\prime}, E_{021}=\left(q+q^{-1}\right)^{-1} E_{021}^{\prime}$ and $E_{031}=\left(q^{2}+1+q^{-2}\right)^{-1} E_{031}^{\prime}$. Otherwise, put $E_{\alpha+\alpha_{i}}=E_{\alpha+\alpha_{i}}^{\prime}$.

(iii) For $\alpha, \beta \in \Phi_{+}^{\text {red }}$ such that $g(\alpha)=g(\beta), \alpha<\beta, h t(\beta)-h t(\alpha) \leq 1$ and $\alpha+\beta \in \Phi_{+}^{\text {red }}$, we put $E_{\alpha+\beta}^{\prime}=\left[E_{\alpha}, E_{\beta}\right]_{q-(\alpha, \beta)}$. If $A$ is of type $C_{N}$ (resp. $D_{N}, F_{4}$ or $\left.G_{3}\right)$, then $E_{\alpha+\beta}$ is defined by $\left(q+q^{-1}\right)^{-1} E_{\alpha+\beta}^{\prime}$ (resp. $\left(q+q^{-1}\right)^{-1} E_{\alpha+\beta}^{\prime}$, $\left(q^{2}+q^{-2}\right)^{-1} E_{\alpha+\beta}^{\prime}$ or $\left.\left(q^{2}+1+q^{-2}\right)^{-1} E_{\alpha+\beta}^{\prime}\right)$.

5.2. The following lemma will play key role in proving our main results (Theorem 10.6.1). 
Lemma 5.2.1. (i) Let $\alpha \in \Phi_{+, i}^{\mathrm{red}}$ (see 3.2 for this definition) and $j>i$. Then we have:

$$
\left[E_{\alpha}, E_{j}\right]_{q}-\left(\alpha, \alpha_{j}\right)=\sum_{\gamma_{1}, \cdots, \gamma_{u} \in \Phi_{+, i}^{\mathrm{red}(\alpha<)}} c_{\gamma_{1}, \cdots, \gamma_{u}} E_{\gamma_{1}} \cdots E_{\gamma_{u}}
$$

for some $c_{\gamma_{1}, \cdots, \gamma_{u}} \in R$.

(ii) Let $\alpha, \beta \in \Phi_{+, i}^{\mathrm{red}}$. If $\alpha<\beta$, then we have:

$$
\left[E_{\alpha}, E_{\beta}\right]_{q}^{-(\alpha, \beta)}=\sum_{\gamma_{1}, \cdots, \gamma_{u} \in \Phi_{+, i}^{\text {red }}(\alpha<\beta)} c_{\gamma_{1}, \cdots, \gamma_{u}} E_{\gamma_{1}} \cdots E_{\gamma_{u}}
$$

for some $c_{\gamma_{1}, \cdots, \gamma_{u}} \in R$.

(iii) $E_{\alpha}^{2}=0$ if $(\alpha, \alpha)=0$.

Remark 5.2.2. In some special cases, we can show more detailed results than Lemma 5.2.1.

(i) Let $\alpha \in \Phi_{+}^{\text {red }}$ satisfy $c_{\alpha}=1$. Take $\alpha_{i} \in \Pi$ such that $i>g(\alpha)$ and $\alpha+\alpha_{i} \notin \Phi_{+}^{\text {red }}$. Then we have:

$$
\left[E_{\alpha}, E_{i}\right]_{q}-\left(\alpha, \alpha_{i}\right)=0
$$

(ii) Assume that $\alpha, \beta \in \Phi_{+}^{\text {red }}$ satisfies $\alpha \nless \beta$ and $\beta \nless \alpha$. Then we have:

$$
\left[E_{\alpha}, E_{\beta}\right]=0
$$

5.3. Let $\prod_{a \in \Phi_{+}^{\text {red }}}^{<}$denote the product taken with respect to a total order on $\Phi_{+}^{\text {red }}$ compatible with the partial order $<$.

As an immediate consequence of Lemma 5.2.1, we have:

Proposition 5.3.1. The $R$-module $\mathcal{N}_{+}$is generated by the elements

$$
\prod_{\alpha \in \Phi_{+}^{\text {red }}}^{<} E_{\alpha}^{n_{\alpha}} \quad\left(n_{\alpha} \in Z_{+} \text {if }(\alpha, \alpha) \neq 0, n_{\alpha}=0,1 \text { if }(\alpha, \alpha)=0\right) .
$$


5.4. The purpose of $\S 6-9$ below, is to prove Lemma 5.2.1 and Remark 5.2.2.

In $\S 10$, we shall prove that the monomials $\prod_{\alpha \in \Phi_{+}^{\text {red }}}^{<} E_{\alpha}^{n_{\alpha}}$ form an orthogonal basis of $\mathscr{N}_{+}$, which implies $\mathscr{I}_{+}=I_{+}$.

\section{§6. Commutation Relations of Root Vectors of $\mathcal{N}_{+}$ (type $A_{N-1}, B_{N}, C_{N}$ or $D_{N}$ )}

6.1. In this section, we assme that $\mathscr{N}_{+}$is of type $A_{N-1}, B_{N}, C_{N}$ or $D_{N-1}$. Put $\bar{d}_{i}=\left(\bar{\varepsilon}_{i}, \bar{\varepsilon}_{i}\right) \in\{1,-1\} \quad(1 \leq i \leq N)$.

Lemma 6.1.1. The following identities hold in $\mathscr{N}_{+}$.

(i) $\left[E_{\alpha_{1}+\alpha_{j}+\alpha_{k}}, E_{j}\right]=0$ for $\stackrel{i}{\times}-\dot{j}^{j}-{ }^{k} \times(1 \leq j \leq k)$,

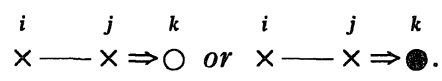

(ii) $\left[E_{\alpha_{1}+\alpha_{j}}, E_{j}\right]_{q-\bar{d}_{j}}=\left[E_{i}, E_{\alpha_{1}+\alpha_{j}}\right]_{q-\bar{d}_{i}}=0$ for $\stackrel{i}{\times} \stackrel{i}{\times} \times(i<j)$.

(iii) $\left[E_{\alpha_{N-2}+\alpha_{N-1}+\alpha_{N}}, E_{N-1}\right]_{q-\bar{d}_{N-1}}$

$$
=\left[E_{\alpha_{N-2}+\alpha_{N-1}+\alpha_{N}}, E_{N}\right]_{q^{-\bar{d}_{N-1}}}=0 \text { for type } D_{N} \text {. }
$$

(iv) $\left[E_{N-1}, E_{\alpha_{N-1}+\alpha_{N}},\right]_{q-\bar{d}_{N-1}}=\left[E_{\alpha_{N-1}+2 \alpha_{N}}, E_{N}\right]_{q-\bar{d}_{N}}=0$ for type $B_{N}$.

(v) $\left[E_{\alpha_{N-1}+\alpha_{N}}, E_{N}\right]_{q^{-2 \bar{d}_{N}}}=0$,

$$
\left[E_{N-1}, E_{2 \alpha_{N-1}+\alpha_{N}}\right]_{q^{-2 \bar{d}_{N-1}}}=0 \text { for type } C_{N} \text {. }
$$

(vi) $\left[E_{\alpha_{N-2}+2 \alpha_{N-1}+\alpha_{N}}, E_{N-1}\right]_{q^{-\bar{d}_{N-1}}}=0$ for type $C_{N}$.

(vii) $\left[E_{2 \alpha_{N-2}+2 \alpha_{N-1}+\alpha_{N}}, E_{N-1}\right]=0\left(p\left(\alpha_{N-2}+\alpha_{N-1}\right)=0\right)$ for type $C_{N}$.

(viii) $\left[E_{\alpha_{N-3}+2 \alpha_{N-2}+2 \alpha_{N-1}+\alpha_{N}}, E_{N-1}\right]=0$ for type $C_{N}$.

Proof. (ii)-(v) These can be proved by direct computations. The proofs 
are immediately obtained from the formula (4.4.3) or Definition 4.2.1 (ii).

(i) This is the defining relation (iii) of Definition 4.2.1 if $p\left(\alpha_{j}\right)=1$. If, $p\left(\alpha_{j}\right)=0$, by Definition 4.2.1 (ii), (4.4.1), and the formula (ii), we have:

$$
\begin{aligned}
& 0= \llbracket E_{i}, E_{j}^{2} E_{k}-\left(q+q^{-1}\right) E_{j} E_{k} E_{j}+E_{k} E_{j}^{2} \rrbracket \\
&=\left\{E_{\alpha_{i}+\alpha_{j}} E_{j}+q^{\bar{d}_{j}} E_{j} E_{\alpha_{i}+\alpha_{j}}\right\} E_{k}-\left(q+q^{-1}\right) E_{\alpha_{i}+\alpha_{j}} E_{k} E_{j} \\
& \quad-(-1)^{p\left(\alpha_{i}\right) p\left(\alpha_{k}\right)}\left(q+q^{-1}\right) q^{\bar{d}_{j}} E_{j} E_{k} E_{\alpha_{i}+\alpha_{j}} \\
& \quad+(-1)^{p\left(\alpha_{i}\right) p\left(\alpha_{k}\right)} E_{k}\left\{E_{\alpha_{i}+\alpha_{j}} E_{j}+q^{\bar{d}_{j}} E_{j} E_{\alpha_{i}+\alpha_{j}}\right\} \\
&=\left(q+q^{-1}\right) E_{j} E_{\alpha_{i}+\alpha_{j}} E_{k}-\left(q+q^{-1}\right) E_{\alpha_{i}+\alpha_{j}} E_{k} E_{j} \\
& \quad-(-1)^{p\left(\alpha_{i}\right) p\left(\alpha_{k}\right)}\left(q+q^{-1}\right) q^{\bar{d}_{j}} E_{j} E_{k} E_{\alpha_{i}+\alpha_{j}} \\
& \quad+(-1)^{p\left(\alpha_{i}\right) p\left(\alpha_{k}\right)} q^{\bar{d}_{j}}\left(q+q^{-1}\right) E_{k} E_{\alpha_{i}+\alpha_{j}} E_{j} \\
&=-\left(q+q^{-1}\right)\left[E_{\alpha_{i}+\alpha_{j}+\alpha_{k}}, E_{j}\right] .
\end{aligned}
$$

(vi) The case $p\left(\alpha_{N-1}\right)=1$ follows from (4.4.3) since, in this case, we have $E_{N-1}^{2}=0$. Assume $p\left(\alpha_{N-1}\right)=0$. By using the facts $p\left(\alpha_{N-1}\right)=p\left(\alpha_{N}\right)=0$ and $\bar{d}_{N-1}=\bar{d}_{N}$, and, by using Definition 4.2.1 (i-ii), (4.4.1) and (ii), we have:

$$
\begin{aligned}
0= & \llbracket E_{N-2},\left\{E_{N-1}^{3} E_{N}-\left(q^{2}+1+q^{-2}\right) E_{N-1}^{2} E_{N} E_{N-1}\right. \\
& \left.+\left(q^{2}+1+q^{-2}\right) E_{N-1} E_{N} E_{N-1}^{2}-E_{N} E_{N-1}^{3}\right\} \rrbracket \\
= & \left(q^{2}+1+q^{-2}\right)\left\{E_{N-1}^{2} E_{\alpha_{N-2}+\alpha_{N-1}} E_{N}-q^{2 \bar{d}_{N-1}} E_{N-1}^{2} E_{N} E_{\alpha_{N-2}+\alpha_{N-1}}\right. \\
& -\left(q+q^{-1}\right) E_{N-1} E_{\alpha_{N-2}+\alpha_{N-1}} E_{N} E_{N-1} \\
& +q^{2 \bar{d}_{N-1}}\left(q+q^{-1}\right) E_{N-1} E_{N} E_{\alpha_{N-2}+\alpha_{N-1}} E_{N-1} \\
& \left.+E_{\alpha_{N-2}+\alpha_{N-1}} E_{N} E_{N-1}^{2}-q^{2 \bar{d}_{N-1}} E_{N} E_{\alpha_{N-2}+\alpha_{N-1}} E_{N-1}^{2}\right\} \\
= & \left(q^{2}+1+q^{-2}\right)\left\{E_{N-1}^{2} E_{\alpha_{N-2}+\alpha_{N-1} \alpha_{N}}\right. \\
& \left.-\left(q+q^{-1}\right) E_{N-1} E_{\alpha_{N-2}+\alpha_{N-1}+\alpha_{N}} E_{N-1}+E_{\alpha_{N-2}+\alpha_{N-1}+\alpha_{N}} E_{N-1}^{2}\right\} \\
= & \left(q^{2}+1+q^{-2}\right) \llbracket E_{\alpha_{N-2}+2 \alpha_{N-1}+\alpha_{N}}, E_{N-1} \rrbracket .
\end{aligned}
$$


(vii) This is the defining relation (vi) of Definition 4.2.1 if $p\left(\alpha_{N-1}\right)=1$. Assume $p\left(\alpha_{N-1}\right)=0$. By (ii), we easily see $\llbracket E_{N-2}, E_{\alpha_{N-2}+\alpha_{N-1}+\alpha_{N}} \rrbracket=0$. By (vi), similarly to the proof of (i), we have:

$$
\begin{aligned}
0= & \llbracket E_{N-2}, \llbracket E_{\alpha_{N-2}+2 \alpha_{N-1}+\alpha_{N}}, E_{N-1} \rrbracket \rrbracket \\
= & \llbracket E_{N-2}\left\{E_{\alpha_{N-2}+\alpha_{N-1}+\alpha_{N}} E_{N-1}^{2}-\left(q+q^{-1}\right) E_{N-1} E_{\alpha_{N-2}+\alpha_{N-1}+\alpha_{N}} E_{N-1}\right. \\
& \left.+E_{N-1}^{2} E_{\alpha_{N-2}+\alpha_{N-1}+\alpha_{N}}\right\} \rrbracket \\
= & \left(q+q^{-1}\right)\left\{E_{N-1} E_{\alpha_{N-2}+\alpha_{N-1}} E_{\alpha_{N-2}+\alpha_{N-1}+\alpha_{N}}\right. \\
& -E_{N-1} E_{\alpha_{N-2}+\alpha_{N-1}+\alpha_{N}} E_{\alpha_{N-2}+\alpha_{N-1}}-E_{\alpha_{N-2}+\alpha_{N-1}} E_{\alpha_{N-2}+\alpha_{N-1}+\alpha_{N}} E_{N-1} \\
& \left.+E_{\alpha_{N-2}+\alpha_{N-1}+\alpha_{N}} E_{\alpha_{N-2}+\alpha_{N-1}} E_{N-1}\right\} \\
= & -\left(q+q^{-1}\right)^{2}\left[E_{2 \alpha_{N-2}+2 \alpha_{N-1}+\alpha_{N}}, E_{N-1}\right] .
\end{aligned}
$$

(viii) If $p\left(\alpha_{N-2}\right)=0$ and, $p\left(\alpha_{N-1}\right)=1$, then (viii) is defining relation (vi) of Definition 4.2.1. Next, we assume $p\left(\alpha_{N-1}\right)=0$. Note that, by (i) and (vi), we have

$$
\begin{aligned}
& \llbracket E_{\alpha_{N-3}+\alpha_{N-2}+\alpha_{N-1}+\alpha_{N}}, E_{N-2} \rrbracket=0, \\
& \llbracket E_{\alpha_{N-3}+\alpha_{N-2}+2 \alpha_{N-1}+\alpha_{N}}, E_{N-1} \rrbracket=0
\end{aligned}
$$

respectively. Hence, by (4.4.5) and (ii), we have:

$$
\begin{aligned}
0= & \llbracket \llbracket \llbracket\left[E_{\alpha_{N-3}+\alpha_{N-2}+\alpha_{N-1}+\alpha_{N}}, E_{N-2} \rrbracket, E_{N-1} \rrbracket, E_{N-1} \rrbracket\right. \\
= & \left(1+(-1)^{p\left(\alpha_{N}-1\right)} q^{-\bar{d}_{N-1}-\bar{d}_{N}}\right) \\
& \left\{(-1)^{p\left(\alpha_{N}-1\right) p\left(\alpha_{N}-2\right)} q^{\bar{d}_{N-1}} E_{\alpha_{N-3}+\alpha_{N-2}+2 \alpha_{N-1}+\alpha_{N}} E_{\alpha_{N-2}+\alpha_{N-1}}\right. \\
& -(-1)^{p\left(\alpha_{N-3}+\alpha_{N-2}+\alpha_{N-1}+\alpha_{N}\right) p\left(\alpha_{N-2}+\alpha_{N-1}\right)} q^{\bar{d}_{N}} \\
& \left.E_{\alpha_{N-2}+\alpha_{N-1}} E_{\alpha_{N-3}+\alpha_{N-2}+2 \alpha_{N-1}+\alpha_{N}}\right\} .
\end{aligned}
$$

Using $p\left(\alpha_{N-1}\right)=0$ and $\bar{d}_{N-1}=\bar{d}_{N}$, the right hand side equals 


$$
\left(q+q^{-1}\right) \llbracket E_{\alpha_{N-3}+\alpha_{N-2}+2 \alpha_{N-1}+\alpha_{N}}, \llbracket E_{N-2}, E_{N-1} \rrbracket \rrbracket .
$$

Here we used the identity $E_{\alpha_{N-2}+\alpha_{N-1}}=\llbracket E_{N-2}, E_{N-1} \rrbracket$. Hence, by (4.4.2) and (6.1.3), this equals

(6.1.4) $\quad\left(q+q^{-1}\right)\left[E_{\alpha_{N-3}+2 \alpha_{N-2}+2 \alpha_{N-1}+\alpha_{N}}, E_{N-1}\right]$.

Finally, we assume $p\left(\alpha_{N-2}\right)=p\left(\alpha_{N-1}\right)=1$. By (i) and (ii), we have:

$$
\llbracket E_{\alpha_{N-3}+\alpha_{N-2}+\alpha_{N-1}}, E_{\alpha_{N-2}+\alpha_{N-1}} \rrbracket=0 .
$$

From this, we have:

$$
\begin{aligned}
& \llbracket E_{N-3}, E_{2 \alpha_{N-2}+2 \alpha_{N-1}+\alpha_{N}} \rrbracket \\
= & \left(q+q^{-1}\right)^{-1} \llbracket E_{N-3},\left\{E_{\alpha_{N-2}+\alpha_{N-1}}^{2} E_{N}\right. \\
& \left.-\left(1+q^{2 \bar{d}_{N}}\right) E_{\alpha_{N-2}+\alpha_{N-1}} E_{N} E_{\alpha_{N-2}+\alpha_{N-1}}+q^{2 \bar{d}_{N}} E_{N} E_{\alpha_{N-2}+\alpha_{N-1}}^{2}\right\} \rrbracket \\
= & \left(q+q^{-1}\right)^{-1}\left\{\left(q+q^{-1}\right) E_{\alpha_{N-2}+\alpha_{N-1}} E_{\alpha_{N-3}+\alpha_{N-2}+\alpha_{N-1}} E_{N}\right. \\
& -\left(q+q^{-1}\right) q^{\bar{d}_{N}} E_{\alpha_{N-2}+\alpha_{N-1}+\alpha_{N}} E_{N} E_{\alpha_{N-2}+\alpha_{N-1}} \\
& -\left(q+q^{-1}\right) q^{\bar{d}_{N-2}+\bar{d}_{N}} E_{\alpha_{N-2}+\alpha_{N-1}} E_{N} E_{\alpha_{N-2}+\alpha_{N-1}+\alpha_{N}} \\
& \left.+\left(q+q^{-1}\right) q^{2 \bar{d}_{N-2}+\bar{d}_{N}} E_{N} E_{\alpha_{N-2}+\alpha_{N-1}+\alpha_{N}} E_{\alpha_{N-2}+\alpha_{N-1}}\right\} \\
= & \llbracket E_{\alpha_{N-2}+\alpha_{N-1}} E_{\alpha_{N-3}+\alpha_{N-2}+\alpha_{N-1}+\alpha_{N}} \rrbracket .
\end{aligned}
$$

Hence, by Definition 4.2.1 (vi), Lemma 6.1.1 (ii) and (4.4.2), we have:

$$
\begin{aligned}
0 & =\llbracket\left[E_{N-3}, \llbracket E_{2 \alpha_{N-2}+2 \alpha_{N-1}+\alpha_{N}}, E_{N-1} \rrbracket \rrbracket\right. \\
& =\llbracket \llbracket\left[E_{N-3}, E_{2 \alpha_{N-2}+2 \alpha_{N-1}+\alpha_{N}} \rrbracket, E_{N-1} \rrbracket\right. \\
& =\llbracket \llbracket E_{\alpha_{N-2}+\alpha_{N-1}}, E_{\alpha_{N-3}+\alpha_{N-2}+\alpha_{N-1}+\alpha_{N}} \rrbracket, E_{N-1} \rrbracket \\
& =-\left[E_{\alpha_{N-3}+\alpha_{N-2}+2 \alpha_{N-1}+\alpha_{N}}, E_{\alpha_{N-2}+\alpha_{N-1}}\right] .
\end{aligned}
$$


But, by (6.1.3) and (4.4.2), this equals

$$
\left[E_{\alpha_{N-3}+2 \alpha_{N-2}+2 \alpha_{N-1}+\alpha_{N}}, E_{N-1}\right]
$$

Hence we get the desired formula.

6.2. Similarly to the proof of the formula (i) of Lemma 6.1.1, we obtain the following lemma.

Lemma 6.2.1. For $\stackrel{i}{\times}-\stackrel{j}{\times}-{ }^{k} \times(i<j<k), \times-\times \Rightarrow$ or $\stackrel{i}{\times}-\stackrel{i}{x} \Rightarrow \stackrel{k}{*}$, the following identities hold:

$$
\begin{aligned}
& {\left[\left[\left[E_{i}, E_{j}\right]_{q^{\left(\alpha_{i}, \alpha_{j}\right)}}, E_{k}\right]_{q^{\left(\alpha_{i}+\alpha_{j}, \alpha_{k}\right)}}, E_{j}\right]=0,} \\
& {\left[E_{\alpha_{i}+\alpha_{j}},\left[E_{j}, E_{k}\right]_{q\left(\alpha_{j}, \alpha_{k}\right)}\right]=0 .}
\end{aligned}
$$

\section{3.}

Lemma 6.3.1. For $1 \leq i \leq N-1$, we have the following identities:

(i) For type $B_{N}$, we have:

$$
\left[E_{\bar{\varepsilon}_{i}}, E_{\bar{\varepsilon}_{i}+\bar{\varepsilon}_{N}}\right]_{q}-\bar{d}_{i}=0 .
$$

(ii) For type $C_{N}$, we have:

$$
\left[E_{2 \bar{\varepsilon}_{i}}, E_{\bar{\varepsilon}_{i}+\bar{\varepsilon}_{N}}\right]_{q-2 \bar{d}_{i}}=0 \quad\left(p\left(\bar{\varepsilon}_{i}-\bar{\varepsilon}_{N}\right)=0\right) .
$$

In the formulas (iii)-(ix) below, we assume that $A$ is of type $D_{N}$.

(iii) $\left[E_{\bar{\varepsilon}_{i}-\bar{\varepsilon}_{N}}, E_{\bar{\varepsilon}_{i}+\bar{\varepsilon}_{N}}\right]=0 \quad\left(p\left(\bar{\varepsilon}_{i}-\bar{\varepsilon}_{N}\right)=0\right)$,

(iv) $\left[E_{\bar{\varepsilon}_{i}-\bar{\varepsilon}_{N}}, E_{\bar{\varepsilon}_{i}+\bar{\varepsilon}_{N-1}}\right]_{q-\bar{d}_{i}}=0$,

(v) $\left[E_{\bar{\varepsilon}_{i}+\bar{\varepsilon}_{N}}, E_{\bar{\varepsilon}_{i}+\bar{\varepsilon}_{N-1}}\right]_{q}-\bar{d}_{i}=0$,

(vi) $\left[E_{2 \bar{\varepsilon}_{i}}, E_{N-1}\right]=0 \quad\left(p\left(\bar{\varepsilon}_{i}-\bar{\varepsilon}_{N}\right)=1\right)$,

(vii) $\left[E_{2 \bar{\varepsilon}_{i}}, E_{N}\right]=\bar{d}_{N}\left(q-q^{-1}\right) E_{\bar{\varepsilon}_{i}+\bar{\varepsilon}_{N}-1} E_{\bar{\varepsilon}_{i}+\bar{\varepsilon}_{N}} \quad\left(p\left(\bar{\varepsilon}_{i}-\bar{\varepsilon}_{N}\right)=1\right)$,

(viii) $\left[E_{\bar{\varepsilon}_{i}-\bar{\varepsilon}_{N}}, E_{2 \bar{\varepsilon}_{i}}\right]_{q-2 \bar{d}_{i}}=0\left(p\left(\bar{\varepsilon}_{i}-\bar{\varepsilon}_{N}\right)=1\right)$, 
(ix) $\left[E_{2 \bar{\varepsilon}_{i}}, E_{\bar{\varepsilon}_{i}+\bar{\varepsilon}_{N}}\right]_{q^{-2} \bar{d}_{i}}=0\left(p\left(\bar{\varepsilon}_{i}-\bar{\varepsilon}_{N}\right)=1\right)$.

Proof. (i) By Lemma 6.1.1 (i), (ii), (iv), we can easily show

$$
\begin{aligned}
& \llbracket E_{\bar{\varepsilon}_{i}-\bar{\varepsilon}_{N}}, E_{\bar{\varepsilon}_{i}} \rrbracket=0, \\
& \llbracket E_{\bar{\varepsilon}_{i}+\bar{\varepsilon}_{N}}, E_{N} \rrbracket=0 .
\end{aligned}
$$

By (4.4.5), we have:

$$
\begin{aligned}
0= & \llbracket \llbracket \llbracket E_{\bar{\varepsilon}_{i}-\bar{\varepsilon}_{N}}, E_{\bar{\varepsilon}_{i}} \rrbracket, E_{N} \rrbracket, E_{N} \rrbracket \\
= & \left(q^{1 / 2}+q^{-1 / 2}\right)\left(( 1 + ( - 1 ) ^ { p ( \overline { \varepsilon } _ { N } ) } q ^ { - \overline { d } _ { N } } ) \left\{(-1)^{p\left(\bar{\varepsilon}_{i}\right) p\left(\bar{\varepsilon}_{N}\right)} E_{\bar{\varepsilon}_{i}} E_{\bar{\varepsilon}_{i}+\bar{\varepsilon}_{N}}\right.\right. \\
& \left.-(-1)^{p\left(\bar{\varepsilon}_{i}-\bar{\varepsilon}_{N}\right) p\left(\bar{\varepsilon}_{i}+\bar{\varepsilon}_{N}\right)} q^{-\bar{d}_{i}+\bar{d}_{N}} E_{\bar{\varepsilon}_{i}+\bar{\varepsilon}_{N}} E_{\bar{\varepsilon}_{i}}\right\} \\
& \left.+E_{\bar{\varepsilon}_{i}+\bar{\varepsilon}_{N}} E_{\bar{\varepsilon}_{i}}-(-1)^{p\left(\bar{\varepsilon}_{i}-\bar{\varepsilon}_{N}\right) p\left(\bar{\varepsilon}_{i}+\bar{\varepsilon}_{N}\right)} q^{-\bar{d}_{i}} E_{\bar{\varepsilon}_{i}} E_{\bar{\varepsilon}_{i}+\bar{\varepsilon}_{N}}\right) \\
= & \left(q^{1 / 2}+q^{-1 / 2}\right)\left(-\left((-1)^{p\left(\bar{\varepsilon}_{i}-\bar{\varepsilon}_{N}\right)} q^{-\bar{d}_{i}}-(-1)^{p\left(\bar{\varepsilon}_{N}\right)}-q^{-\bar{d}_{N}}\right)\right. \\
& (-1)^{p\left(\bar{\varepsilon}_{i}-\bar{\varepsilon}_{N}\right) p\left(\bar{\varepsilon}_{N}\right)} E_{\bar{\varepsilon}_{i}} E_{\bar{\varepsilon}_{i}+\bar{\varepsilon}_{N}} \\
& \left.+\left((-1)^{p\left(\bar{\varepsilon}_{i}-\bar{\varepsilon}_{N}\right)}-q^{-\bar{d}_{i}+\bar{d}_{N}}-(-1)^{p\left(\bar{\varepsilon}_{N}\right)} q^{-\bar{d}_{i}}\right)(-1)^{p\left(\bar{\varepsilon}_{i}-\bar{\varepsilon}_{N}\right)} E_{\bar{\varepsilon}_{i}+\bar{\varepsilon}_{N}} E_{\bar{\varepsilon}_{i}}\right) \\
= & -\left(q^{1 / 2}+q^{-1 / 2}\right)\left((-1)^{p\left(\bar{\varepsilon}_{i}-\bar{\varepsilon}_{N}\right)} q^{-\bar{d}_{i}}-(-1)^{p\left(\bar{\varepsilon}_{N}\right)}-q^{-\bar{d}_{N}}\right) \\
& (-1)^{p\left(\bar{\varepsilon}_{i}-\bar{\varepsilon}_{N}\right) p\left(\bar{\varepsilon}_{N}\right)} \llbracket E_{\bar{\varepsilon}_{i}} E_{\bar{\varepsilon}_{i}+\bar{\varepsilon}_{N}} \rrbracket .
\end{aligned}
$$

(ii) This can be proved similarly to (i).

(iii) By Lemma 6.1.1 (i)-(ii), it can be easily proved that

$$
\begin{aligned}
& 0=\llbracket E_{\bar{\varepsilon}_{i}-\bar{\varepsilon}_{N-1}}, E_{\bar{\varepsilon}_{i}-\bar{\varepsilon}_{N}} \rrbracket, \\
& 0=\llbracket E_{\bar{\varepsilon}_{i}-\bar{\varepsilon}_{N-1}}, E_{\bar{\varepsilon}_{i}+\bar{\varepsilon}_{N}} \rrbracket .
\end{aligned}
$$

If $p\left(\bar{\varepsilon}_{i}-\bar{\varepsilon}_{N-1}\right)=0$ (resp. $p\left(\bar{\varepsilon}_{i}-\bar{\varepsilon}_{N-1}\right)=1$ ), by Definition 4.2 .1 . (i)-(ii) (resp. (v)), we have:

$$
0=\llbracket E_{\bar{\varepsilon}_{i}-\bar{\varepsilon}_{N}}, E_{N} \rrbracket-\llbracket E_{\bar{\varepsilon}_{i}+\bar{\varepsilon}_{N}}, E_{N-1} \rrbracket .
$$


Hence, by (6.3.2-3) and (4.4.2), we have:

$$
\begin{aligned}
0= & \llbracket E_{\bar{\varepsilon}_{i}-\bar{\varepsilon}_{N-1}},\left\{\llbracket E_{\bar{\varepsilon}_{i}-\bar{\varepsilon}_{N}}, E_{N} \rrbracket-\llbracket E_{\bar{\varepsilon}_{i}+\bar{\varepsilon}_{N}}, E_{N-1} \rrbracket\right\} \rrbracket \\
= & \left\{(-1)^{p\left(\bar{\varepsilon}_{i}-\bar{\varepsilon}_{N}-1\right) p\left(\bar{\varepsilon}_{i}-\bar{\varepsilon}_{N}\right)} q^{-\bar{d}_{i}}\right. \\
& \left.+(-1)^{p\left(\bar{\varepsilon}_{i}+\bar{\varepsilon}_{N}\right) p\left(\bar{\varepsilon}_{N-1}-\bar{\varepsilon}_{N}\right)} q^{\bar{d}_{N}}\right\} E_{\bar{\varepsilon}_{i}+\bar{\varepsilon}_{N}} E_{\bar{\varepsilon}_{i}-\bar{\varepsilon}_{N}} \\
& -\left\{(-1)^{p\left(\bar{\varepsilon}_{i}-\bar{\varepsilon}_{N}\right) p\left(\bar{\varepsilon}_{N-1}+\bar{\varepsilon}_{N}\right)} q^{\bar{d}_{N}}\right. \\
& \left.+(-1)^{p\left(\bar{\varepsilon}_{i}-\bar{\varepsilon}_{N}-1\right) p\left(\bar{\varepsilon}_{i}+\bar{\varepsilon}_{N}\right)} q^{-\bar{d}_{i}}\right\} E_{\bar{\varepsilon}_{i}+\bar{\varepsilon}_{N}} E_{\bar{\varepsilon}_{i}-\bar{\varepsilon}_{N}} .
\end{aligned}
$$

Since $p\left(\bar{\varepsilon}_{i}-\bar{\varepsilon}_{N}\right)=p\left(\bar{\varepsilon}_{i}+\bar{\varepsilon}_{N}\right)=0$ and $\bar{d}_{i}=\bar{d}_{N}$, this equals $\left(q+q^{-1}\right)\left[E_{\bar{\varepsilon}_{i}-\bar{\varepsilon}_{N}}, E_{\bar{\varepsilon}_{i}+\bar{\varepsilon}_{N}}\right]$, which implies (iii).

(iv) By Lemma 6.1.1 (ii), we can easily show:

$$
\llbracket E_{\bar{\varepsilon}_{i}-\bar{\varepsilon}_{N}}, E_{N-1} \rrbracket=0
$$

If $p\left(\bar{\varepsilon}_{i}-\bar{\varepsilon}_{N}\right)=0$, by (iii), (6.3.4) and (4.4.2), we have:

$$
0=\llbracket \llbracket E_{\bar{\varepsilon}_{i}-\bar{\varepsilon}_{N}}, E_{\bar{\varepsilon}_{i}+\bar{\varepsilon}_{N}} \rrbracket, E_{N-1} \rrbracket=\llbracket E_{\bar{\varepsilon}_{i}-\bar{\varepsilon}_{N}}, E_{\bar{\varepsilon}_{i}+\bar{\varepsilon}_{N-1}} \rrbracket .
$$

By (6.3.2) and (6.3.4), we have:

$$
0=(-1)^{p\left(\bar{\varepsilon}_{i}-\bar{\varepsilon}_{N}\right) p\left(\bar{\varepsilon}_{N}-1-\bar{\varepsilon}_{N}\right)} q^{-\bar{d}_{N}} E_{\bar{\varepsilon}_{i}-\bar{\varepsilon}_{N}}^{2}-(-1)^{p\left(\bar{\varepsilon}_{i}-\bar{\varepsilon}_{N}-1\right) p\left(\bar{\varepsilon}_{i}-\bar{\varepsilon}_{N}\right)} q^{-\bar{d}_{i}} E_{\bar{\varepsilon}_{i}-\bar{\varepsilon}_{N}}^{2}
$$

If $p\left(\bar{\varepsilon}_{i}-\bar{\varepsilon}_{N}\right)=1$, this implies $0=\left(q^{\bar{d}_{N}}+q^{-\bar{d}_{N}}\right) E_{\bar{\varepsilon}_{i}-\bar{\varepsilon}_{N}}^{2}$. Hence, by (4.4.3), we have (iv).

The formula (v) can be proved quite similarly to (iv).

The formula (vi) - (ix) can be easily proved by using (iii)-(v).

\section{4.}

Lemma 6.4.1. The following identities hold in $\mathcal{N}_{+}$.

(i) $\left[E_{\alpha_{N-3}+2 \alpha_{N-2}+\alpha_{N-1}+\alpha_{N}}, E_{N}\right]=0$ for type $D_{N}$.

(ii) $\left[E_{\alpha_{N-3}+2 \alpha_{N-2}+\alpha_{N-1}+\alpha_{N}}, E_{N-1}\right]=0$ for type $D_{N}$. 
(iii) $\left[E_{\alpha_{N-2}+2 \alpha_{N-1}+2 \alpha_{N}}, E_{N}\right]=0$ for type $B_{N}$.

(iv) $\left[E_{\alpha_{N-2}+2 \alpha_{N-1}+\alpha_{N}}, E_{N}\right]=0$ for type $C_{N}$.

Proof. In the proof, we may assume that $N=4$. Put $E_{\text {abcd }}=$ $E_{a \alpha_{1}+b \alpha_{2}+c \alpha_{3}+d \alpha_{4}}$. Denote $p\left(\alpha_{i}\right)\left(\operatorname{resp} . p\left(\alpha_{i_{1}}+\cdots+\alpha_{i_{r}}\right)\right)$ by $p(i)\left(\operatorname{resp} . p\left(i_{1} \cdots i_{r}\right)\right)$.

(i) We are in one of the following three cases.

(1) $p(3)=p(4)=0$,

(2) $p(2)=0$,

(3) $p(2)=p(3)=p(4)$.

First consider the case (1). From Lemma 6.1.1 (i) and (4.4.5), we have:

$$
\begin{aligned}
0= & \llbracket \llbracket \llbracket \llbracket E_{1110}, E_{2} \rrbracket, E_{4} \rrbracket, E_{4} \rrbracket \\
= & \llbracket E_{1110}, \llbracket E_{0101}, E_{4} \rrbracket \rrbracket \\
& +\left(1+(-1)^{p(4)} q^{-\bar{d}_{3}-\bar{d}_{4}}\right)\left\{(-1)^{p(2) p(4)} q^{\bar{d}_{3}} E_{1111} E_{0101}\right. \\
& \left.-(-1)^{p(123) p(24)} q^{\bar{d}_{4}} E_{0101} E_{1111}\right\} \\
& +q^{2 \bar{d}_{4}} \llbracket E_{1111}, E_{4} \rrbracket E_{2}-(-1)^{p(123) p(2)} E_{2} \llbracket E_{1111} E_{4} \rrbracket .
\end{aligned}
$$

By Definition 4.2 .1 , we can easily show $\llbracket E_{0101}, E_{4} \rrbracket=\llbracket E_{1111}, E_{4} \rrbracket=0$. Since $p(3)=p(4)=0$ and $\bar{d}_{3}=\bar{d}_{4}$, the right hand side equals

$$
\left(q+q^{-1}\right)\left[E_{1111}, E_{0101}\right]
$$

Using (4.4.2) and Lemma 6.1.1 (iii), we have:

$$
\left[E_{1111}, E_{0101}\right]=\left[E_{1111},\left[E_{2}, E_{4}\right]_{q^{\bar{q}_{3}}}\right]=\left[E_{1211}, E_{4}\right]
$$

Hence we have $\left[E_{1211}, E_{4}\right]=0$.

The case (2); by Lemma 6.3.1(v), $\left[E_{0101}, E_{0111}\right]_{q^{-\bar{d}_{2}}}=0$.

Similarly to the case (1), by Lemma 6.1 .1 (i) and (4.4.2), (4.4.4), we have: 


$$
\begin{aligned}
0= & \llbracket \llbracket \llbracket E_{1101}, E_{2} \rrbracket, E_{3} \rrbracket, E_{4} \rrbracket-\llbracket E_{1}, \llbracket E_{0101}, E_{0111} \rrbracket \rrbracket \\
= & \left\{\llbracket E_{1101}, E_{0111} \rrbracket+(-1)^{p(23) p(4)} q^{\bar{d}_{4}} \llbracket E_{1101}, E_{4} \rrbracket E_{0110}\right. \\
& -(-1)^{p(124) p(23)} q^{\bar{d}_{4}} E_{0110} \llbracket E_{1101}, E_{4} \rrbracket \\
& +(-1)^{p(2) p(3)} q^{\bar{d}_{3}} E_{1111} E_{0101}+(-1)^{p(2) p(34)} q^{2 \bar{d}_{3}} \llbracket E_{1111}, E_{4} \rrbracket E_{2} \\
& -(-1)^{p(124) p(2)} E_{2} \llbracket E_{1111}, E_{4} \rrbracket \\
& \left.-(-1)^{p(124) p(2)}(-1)^{p(1234) p(4)} q^{-\bar{d}_{3}} E_{0101}, E_{1111}\right\} \\
& -\left\{\llbracket E_{1101}, E_{0111} \rrbracket-(-1)^{p(24) p(234)} q^{-\bar{d}_{2}} E_{1111}, E_{0101}\right. \\
& \left.+(-1)^{p(1) p(24)} q^{\bar{d}_{2}} E_{0101} E_{1111}\right\} .
\end{aligned}
$$

By Definition 4.2.1, it can be easily shown that

$$
\llbracket E_{1101}, E_{4} \rrbracket=\llbracket E_{1111}, E_{4} \rrbracket=0 .
$$

Since $p(2)=0, p(3)=p(4)$ and $\bar{d}_{3}=\bar{d}_{2}$, the above equals

$$
\begin{aligned}
& \left\{q^{\bar{d}_{2}} E_{1111} E_{0101}-(-1)^{p(1) p(4)} q^{-\bar{d}_{2}} E_{0101} E_{1111}\right\} \\
+ & \left\{q^{-\bar{d}_{2}} E_{1111} E_{0101}+(-1)^{p(1) p(4)} q^{\bar{d}_{2}} E_{0101} E_{1111}\right\} \\
= & \left(q+q^{-1}\right) \llbracket\left[E_{1111}, E_{0101} \rrbracket .\right.
\end{aligned}
$$

Hence, similarly to (6.4.2), we have $\left[E_{1211}, E_{4}\right]=0$.

Finally assume that we are in the case (3). We can easily show that $E_{0101}^{2} E_{1}-\left(q+q^{-1}\right) E_{0101} E_{1} E_{0101}+E_{1} E_{0101}^{2}=0$ and $E_{0101}^{2} E_{3}-\left(q+q^{-1}\right) E_{0101}$ $E_{3} E_{0101}+E_{3} E_{0101}^{2}=0$. Then, similarly to Lemma 6.1 .1 (i), we can prove our formula.

(ii) The proof of (ii) is quite similar to that of (i).

(iii) The proof is similarly to the case (2) in the proof of (i). By Lemma 6.4.1 (i), we have $\llbracket E_{0011}, E_{0012} \rrbracket=0$. Hence, by (4.4.2) and (4.4.5), we have:

$$
\begin{aligned}
0= & \llbracket \llbracket \llbracket E_{0111}, E_{3} \rrbracket, E_{4} \rrbracket E_{4} \rrbracket \\
& -\left(q^{1 / 2}+q^{-1 / 2}\right) \llbracket E_{2}, \llbracket E_{0011}, E_{0012} \rrbracket \rrbracket \\
= & \left\{\llbracket E_{0111},\left(q^{1 / 2}+q^{-1 / 2}\right) E_{0012} \rrbracket\right. \\
& +\left(1+(-1)^{p(4)} q^{-\bar{d}_{4}}\left((-1)^{p(3) p(4)} q^{\bar{d}_{4}}\left(q^{1 / 2}+q^{-1 / 2}\right) E_{0112} E_{0011}\right.\right.
\end{aligned}
$$




$$
\begin{aligned}
& \left.-(-1)^{p(234) p(34)}\left(q^{1 / 2}+q^{-1 / 2}\right) E_{0011} E_{0112}\right) \\
& +q^{2 \bar{d}_{4}}\left(q^{1 / 2}+q^{-1 / 2}\right) \llbracket E_{0112}, E_{4} \rrbracket E_{3} \\
& \left.+(-1)^{p(234) p(3)}\left(q^{1 / 2}+q^{-1 / 2}\right) E_{3} \llbracket E_{0112}, E_{4} \rrbracket\right\} \\
& -\left(q^{1 / 2}+q^{-1 / 2}\right)\left\{\llbracket E_{0111}, E_{0012} \rrbracket-(-1)^{p(34) p(344)} q^{-\bar{d}_{3}} E_{0112} E_{0011}\right. \\
& \left.+(-1)^{p(2) p(34)} q^{\bar{d}_{3}} E_{0011} E_{0112}\right\}
\end{aligned}
$$

By Lemma 6.1.1 (iv), we can easily show $\llbracket E_{0112}, E_{4} \rrbracket=0$. Hence the above equals

$$
\begin{aligned}
& \left\{\left(1+(-1)^{p(4)} q^{-\bar{d}_{4}}\left((-1)^{p(3) p(4)} q^{\bar{d}_{4}}\left(q^{1 / 2}+q^{-1 / 2}\right) E_{0112} E_{0011}\right.\right.\right. \\
& \left.\left.-(-1)^{p(234) p(34)}\left(q^{1 / 2}+q^{-1 / 2}\right) E_{0011} E_{0112}\right)\right\} \\
& -\left(q^{1 / 2}+q^{-1 / 2}\right)\left\{-(-1)^{p(34) p(3)} q^{-\bar{d}_{3}} E_{0112}, E_{0011}\right. \\
& \left.+(-1)^{p(2) p(34)} q^{\bar{d}_{3}} E_{0011}, E_{0112}\right\} \\
= & \left(q^{1 / 2}+q^{-1 / 2}\right)\left\{(-1)^{p(3) p(4)}\left(q^{\bar{d}_{4}}+(-1)^{p(4)}+(-1)^{p(3)} q^{-\bar{d}_{3}}\right) E_{0112} E_{0011}\right. \\
& -(-1)^{p(234) p(34)}\left(1+(-1)^{p(4)} q^{-\bar{d}_{4}}+(-1)^{p(34)} q^{\bar{d}_{3}}\right) E_{0011} E_{0112} \\
= & \left(q^{1 / 2}+q^{-1 / 2}\right)\left(q^{\bar{d}_{4}}+(-1)^{p(4)}+(-1)^{p(3)} q^{-\bar{d}_{3}}\right)(-1)^{p(3) p(4)} \llbracket E_{0112}, E_{0011} \rrbracket .
\end{aligned}
$$

Hence, similarly to $(6.4 .2)$, we have $\left[E_{0122}, E_{4}\right]=0$.

(iv) By Lemma $6.1 .1(\mathrm{v})$, we have $\left[E_{0011}, E_{4}\right]_{q^{-2 \bar{d}_{4}}}=\left[E_{0111}, E_{4}\right]_{q^{-2 \bar{d}_{4}}}=0$. Therefore, using (4.4.5), we have:

$$
\begin{aligned}
0= & \llbracket \llbracket \llbracket E_{0110}, E_{3} \rrbracket, E_{4} \rrbracket, E_{4} \rrbracket \\
= & \left(1+(-1)^{p(4)} q^{-4 \bar{d}_{4}}\right)\left((-1)^{p(3) p(4)} q^{2 \bar{d}_{4}} E_{0111} E_{0011}\right. \\
& \left.-(-1)^{p(23) p(34)} q^{\bar{d}_{4}} E_{0011}, E_{0111}\right) .
\end{aligned}
$$

Since $p(4)=0$, this equals

$$
\left(q^{2}+q^{-2}\right) \llbracket E_{0111}, E_{0011} \rrbracket .
$$

Hence, by Lemma 6.1.1 (v) and (4.4.2), we have

$$
0=\llbracket E_{0111}, E_{0011} \rrbracket=\llbracket E_{0111}, \llbracket E_{3}, E_{4} \rrbracket \rrbracket=\left[E_{0121}, E_{4}\right]
$$

This completes the proof of Lemma 6.4.1. 


\section{5 .}

Lemma 6.5.1. Let $\mathcal{N}_{+}$be of type $C_{N}$. Let $i \in\{1, \cdots, N-1\}$. We have:

(i) $\left[E_{\bar{\varepsilon}_{i}+\bar{\varepsilon}_{N-1}}, E_{\bar{\varepsilon}_{i}-\bar{\varepsilon}_{N}}\right]=0(1 \leq i \leq N-3)$.

(ii) $\left[E_{2 \bar{\varepsilon}_{i}}, E_{N-1}\right]=0(1 \leq i \leq N-2)$ if $p\left(\bar{\varepsilon}_{i}-\bar{\varepsilon}_{N-1}\right)=0$.

Proof. (i) If $i=N-2$, this is proved easily by using Lemma 6.1 .1 (ii), (vii). Assume $i \leq N-3$. By Lemma 6.1.1 (i)-(ii) (resp. (viii)), we can easily show that

$$
0=\llbracket E_{\bar{\varepsilon}_{i}-\bar{\varepsilon}_{N-2}}, E_{\bar{\varepsilon}_{i}+\bar{\varepsilon}_{N-1}} \rrbracket
$$

(resp.

$$
\left.0=\llbracket E_{\bar{\varepsilon}_{i}+\bar{\varepsilon}_{N-2}}, E_{N-1} \rrbracket\right) \text {. }
$$

Using (4.4.2), from (6.5.3), we obtain:

$$
0=\llbracket E_{\bar{\varepsilon}_{i}+\bar{\varepsilon}_{N-1}}, E_{\bar{\varepsilon}_{N-2}-\bar{\varepsilon}_{N}} \rrbracket .
$$

Hence, using this and (6.5.2), we have (i).

(ii) By Lemma 6.1.1 (ii), we can easily show that $\llbracket E_{\bar{\varepsilon}_{i}-\bar{\varepsilon}_{N}}, E_{N-1} \rrbracket=0$. From this and the formula (i), we can immediately prove (ii).

6.6. Lemma 5.2 .1 (and Remark 5.2.2) for $\Phi_{+}^{\text {red }}$ of type $A_{N-1}, B_{N}, C_{N}$ or $D_{N}$ can be proved using lemmas in 6.1-6.5. In 6.7-6.9 below, this will be done only in some special cases. In the remaining cases, the proof can be done similarly and more easily.

6.7. Proof of Lemma 5.2.1 (i) for $\Phi_{+}^{\mathrm{red}}$ of type $A_{N-1}, B_{N}, C_{N}$ or $D_{N}$. Here we give a proof in the case when $\Phi_{+}^{\text {red }}$ is of type $B_{N}$ and $\alpha=\bar{\varepsilon}_{i}+\bar{\varepsilon}_{k}$ $(i+1<k<N-1)$. The other case can be treated similarly.

Since $\left[E_{i}, E_{j}\right]=0$ if $|i-j| \geq 2$, by (4.4.2), we have

(6.7.1) $E_{\bar{\varepsilon}_{i}}=\left[\left[E_{\bar{\varepsilon}_{i}-\bar{\varepsilon}_{u-1}}, E_{\bar{\varepsilon}_{u-1}-\bar{\varepsilon}_{u+2}}\right]_{q} \bar{d}_{u-1}, E_{\bar{\varepsilon}_{u+2}}\right]_{q} \bar{d}_{u+2}$

if $i<u<N$.

Hence, by Lemma 6.1.1 (i), we have: 


$$
\left[E_{\bar{\varepsilon}_{i}}, E_{u}\right]=0 \text { if } i<u<N
$$

Hence, putting $E_{\bar{\varepsilon}_{k}}^{v}=\left[\left[\cdots\left[E_{N}, E_{N-1}\right]_{q^{\bar{d}_{N}} \cdots, E_{k+1}}\right]_{q^{\bar{d}_{k}+2}}, E_{k}\right]_{q} \bar{q}_{\bar{d}_{k+1}}$, we have:

$$
E_{\bar{\varepsilon}_{i}+\bar{\varepsilon}_{k}}=\left(q^{1 / 2}+q^{-1 / 2}\right)^{-1}\left[E_{\bar{\varepsilon}_{i}}, E_{\bar{\varepsilon}_{k}}^{\vee}\right]
$$

Similarly to (6.7.2), using Lemma 6.2.1 (i), we have:

$$
\left[E_{\bar{\varepsilon}_{k}}^{\vee}, E_{u}\right]=0 \text { if } k<u<N
$$

Hence, we have:

$$
\left[E_{\bar{\varepsilon}_{i}+\bar{\varepsilon}_{k}}, E_{u}\right]=0 \text { if } i<u<k-1 \text { or } k<u<N .
$$

By Definition 5.1.1, we have:

$$
\left[E_{\bar{\varepsilon}_{i}+\bar{\varepsilon}_{k}}, E_{k-1}\right]_{q}{\overline{\bar{d}_{k}}}_{k}=E_{\bar{\varepsilon}_{i}+\bar{\varepsilon}_{k-1}} .
$$

By Definition 4.2.1 (i),(ii), it can be easily shown that

$$
\left[E_{\bar{\varepsilon}_{k}}^{\vee}, E_{k}\right]_{q-\bar{d}_{k}}=0
$$

Hence, we have:

$$
\left[E_{\bar{\varepsilon}_{i}+\bar{\varepsilon}_{k}}, E_{k}\right]_{q-\bar{d}_{k}}=0 \text {. }
$$

By (6.7.2), putting

$$
\begin{gathered}
E_{\bar{\varepsilon}_{k}-\bar{\varepsilon}_{N-1}}^{\vee}=\left[\left[\cdots\left[E_{N-2}, E_{N-3}\right]_{q} \bar{d}_{N-3} \cdots, E_{k+1}\right]_{q}{\overline{\bar{d}_{k+2}}}_{\bar{d}_{k}}, E_{k}\right]_{q^{\bar{d}_{k+1}}} \text {, we have: } \\
E_{\bar{\varepsilon}_{i}+\bar{\varepsilon}_{k}}=\left[\left[E_{\bar{\varepsilon}_{t}-\bar{\varepsilon}_{k-1}}, E_{\bar{\varepsilon}_{k-1}+\bar{\varepsilon}_{N-1}}\right]_{q} \bar{d}_{\bar{d}_{k-1}}, E_{\bar{\varepsilon}_{k}-\bar{\varepsilon}_{N-1}}^{\vee}\right]_{q} \bar{d}_{N-1} .
\end{gathered}
$$

Hence, by Lemma 6.4.1 (iii), we have:

$$
\left[E_{\bar{\varepsilon}_{i}+\bar{\varepsilon}_{k}}, E_{N}\right]=0
$$

as required. Remark 5.2.2 (i) also follows from this and (6.7.5). 
6.8. Proof of Lemma 5.2 .1 (ii) for $\Phi_{+}^{\text {red }}$ of type $A_{N-1}, B_{N}, C_{N}$ or $D_{N}$. Here we give a proof in the case when $\Phi_{+}^{\text {red }}$ is of type $B_{N}$ and $\beta=\bar{\varepsilon}_{i}+\bar{\varepsilon}_{k}$ $(i+1<k<N-1)$.

By Lemma 6.1.1 (ii) and (6.7.2), we have:

$$
\left[E_{\bar{\varepsilon}_{i}-\bar{\varepsilon}_{u}}, E_{\bar{\varepsilon}_{i}}\right]_{q}-\bar{d}_{i}=0 \text { if } i<u<N \text {. }
$$

Similarly to (6.7.1), we have

$$
\begin{aligned}
& E_{\bar{\varepsilon}_{i}-\bar{\varepsilon}_{u}}=\left[\left[E_{\bar{\varepsilon}_{i}-\bar{\varepsilon}_{k-1}}, E_{\bar{\varepsilon}_{k-1}+\bar{\varepsilon}_{k+1}}\right]_{q}{\overline{\bar{k}_{k-1}}}_{1}, E_{\bar{\varepsilon}_{k+1}-\bar{\varepsilon}_{u}}\right]_{q} \bar{d}_{k+1}, \\
& E_{\bar{\varepsilon}_{k}}^{\vee}=\left[E_{\bar{\varepsilon}_{k+2}},\left[E_{k+1}, E_{k}\right]_{q^{\bar{k}_{k}}}\right]_{q^{\bar{d}_{k+2}}} .
\end{aligned}
$$

if $i<k<u \leq N$.

Hence, by Lemma 6.2.1 (ii) and (6.7.4), we have:

$$
\left[E_{\bar{\varepsilon}_{i}-\bar{\varepsilon}_{u}}, E_{\bar{\varepsilon}_{k}}^{\vee}\right] \text { if } k<u \leq N
$$

By (6.7.3) and (6.8.1-2), we have:

$$
\left[E_{\bar{\varepsilon}_{i}-\bar{\varepsilon}_{u}}, E_{\bar{\varepsilon}_{i}+\bar{\varepsilon}_{k}}\right]_{q-\bar{d}_{i}}=0
$$

if $i<u<k-1$ or $k<u \leq N$.

If $\alpha=\bar{\varepsilon}_{i}-\bar{\varepsilon}_{k}$, then we can inductively show the formula by using the following fact.

$$
\begin{aligned}
& {\left[E_{\bar{\varepsilon}_{i}-\bar{\varepsilon}_{k}}, E_{\bar{\varepsilon}_{i}+\bar{\varepsilon}_{k}}\right]_{q} \bar{d}_{k}-\bar{d}_{i}} \\
& =\left[E_{\bar{\varepsilon}_{i}-\bar{\varepsilon}_{k}},\left[E_{\bar{\varepsilon}_{i}+\bar{\varepsilon}_{k+1}}, E_{k}\right]_{q} \bar{d}_{k+1}\right]_{q}{\overline{\bar{d}_{k}}}-\bar{d}_{i} \\
& =(-1)^{p\left(\bar{\varepsilon}_{i}+\bar{\varepsilon}_{k+1}\right) p\left(\bar{\varepsilon}_{k}-\bar{\varepsilon}_{k+1}\right)}\left(q^{-\bar{d}_{k+1}}-q^{\bar{d}_{k+1}}\right) E_{\bar{\varepsilon}_{i}-\bar{\varepsilon}_{k+1}} E_{\bar{\varepsilon}_{i}+\bar{\varepsilon}_{k+1}} \\
& -(-1)^{p\left(\bar{\varepsilon}_{i}+\bar{\varepsilon}_{k+1}\right) p\left(\bar{\varepsilon}_{k}-\bar{\varepsilon}_{k+1}\right)} q^{-\bar{d}_{k+1}}\left[E_{\bar{\varepsilon}_{i}-\bar{\varepsilon}_{k+1}}, E_{\bar{\varepsilon}_{i}+\bar{\varepsilon}_{k+1}}\right]_{q}{\overline{d_{k}+1}}-\bar{d}_{i} .
\end{aligned}
$$

Since $E_{\bar{\varepsilon}_{i}}=\left[E_{\bar{\varepsilon}_{i}-\bar{\varepsilon}_{N}}, E_{N}\right]_{q \bar{d}_{N}}$ by $(6.7 .7)$ and (6.8.3), we have:

$$
\left[E_{\bar{\varepsilon}_{i}}, E_{\bar{\varepsilon}_{i}+\bar{\varepsilon}_{k}}\right]_{q-\bar{d}_{i}}=0 \text {. }
$$


By (6.7.5), (6.7.7) and (6.8.4), we have

$$
\left[E_{\bar{\varepsilon}_{i}+\bar{\varepsilon}_{u}}, E_{\bar{\varepsilon}_{i}+\bar{\varepsilon}_{k}}\right]_{q}-\bar{d}_{i}=0 \text { if } k<u \leq N .
$$

Other cases can be shown similarly.

6.9. Proof of Lemma 5.2.1 (iii) for $\Phi_{+}^{\text {red }}$ of type $A_{N-1}, B_{N}, C_{N}$ or $D_{N}$. Here we give a proof in the case when $\Phi_{+}^{\text {red }}$ is of type $B_{N}$ and $\alpha=\bar{\varepsilon}_{i}+\bar{\varepsilon}_{k}(i+1<k<N-1)$.

By Definition 5.1.1 and (6.7.6), (6.8.5), we have:

$$
\begin{aligned}
0 & =\left[\left[E_{\bar{\varepsilon}_{i}+\bar{\varepsilon}_{k+1}}, E_{k}\right]_{q} \bar{d}_{k}, E_{\bar{\varepsilon}_{i}+\bar{\varepsilon}_{k}}\right]_{q} \bar{d}_{k_{k}}-\bar{d}_{i} \\
& =\left[E_{\bar{\varepsilon}_{i}+\bar{\varepsilon}_{K}}, E_{\bar{\varepsilon}_{i}+\bar{\varepsilon}_{k}}\right]_{q_{\bar{d}_{k}}-\bar{d}_{i}} \\
& =\left(1-(-1)^{p\left(\bar{\varepsilon}_{i}+\bar{\varepsilon}_{k}\right)} q^{\bar{d}_{k}-\bar{d}_{i}}\right) E_{\bar{\varepsilon}_{i}+\bar{\varepsilon}_{K}}^{2} .
\end{aligned}
$$

Since $p\left(\bar{\varepsilon}_{i}+\bar{\varepsilon}_{k}\right)=1$, we have $E_{\bar{\varepsilon}_{i}+\bar{\varepsilon}_{K}}^{2}=0$. Other cases can be proved similarly.

\section{§7. Braid Group Actions on Quantized Enveloping Algebras}

7.1. In this section, we briefly explain the braid group action on $U_{h}(G)$ introduced by Lusztig [11] and [12].

Let $\left(\mathscr{E}, \Pi=\left\{\alpha_{1}, \cdots, \alpha_{n}\right\}, p\right)$ be a triple system. Assume that $\Pi$ is the set of the simple roots of a complex simple Lie algebra $G$ and that $p\left(\alpha_{i}\right)=0$ for any $\alpha_{i}$. Put $d_{i}=\left(\alpha_{i}, \alpha_{i}\right) / 2(1 \leq i \leq n)$ and $D=\operatorname{diag}\left(d_{1}, \cdots, d_{n}\right)$. Let $s_{i} \in G L(\mathscr{E})$ be such that $s_{i}(x)=x-\frac{2\left(\alpha_{i}, x\right)}{\left(\alpha_{i}, \alpha_{i}\right)} \alpha_{i}(x \in \mathscr{E})$. Let $\mathscr{W}$ be the Weyl group, i.e., the group generated by the elements $s_{i}(1 \leq i \leq n)$. Let $U_{h}^{\sigma}=U_{h}^{\sigma}((\mathscr{E}, \Pi, p), D)$ be the $h$-adic $R$-Hopf algebra defined in Theorem 2.9.4. Put $F_{i}=E_{i}^{\circ}$. Then Drinfeld's [4] $U_{h}(G)$ is equal to the unital subalgebra of $U_{h}^{\sigma} h$-adically generated by the elements $E_{i}, F_{i}(1 \leq i \leq n), H \in \mathscr{H}$ (see Theorem 2.10.1). We have $U_{h}^{\sigma}=U_{h}(G) \oplus R\langle\sigma\rangle$. We put $U_{h}(\mathscr{E}, \Pi)=U_{h}(G)$.

Let $d_{i}=\frac{\left(\alpha_{i}, \alpha_{i}\right)}{2} \quad(1 \leq i \leq n)$ and $q_{i}=\exp \left(h d_{i}\right)$. Put $K_{i}=\exp \left(h H_{\alpha_{i}}\right)$ and $E_{i}^{(r)}=E_{i}^{r} /[r]_{q_{i}} !, \quad F_{i}^{(r)}=F_{i}^{r} /[r]_{q_{i}} !$ where $[r]_{q_{i}} !=\prod_{v=1}^{r} \frac{q_{i}^{v}-q_{i}^{-v}}{q_{i}-q_{i}^{-1}}$. In [11] and [12], 
Lusztig introduced a braid group action on $U_{h}(\mathscr{E}, \Pi)$.

Proposition 7.1.1. ([11] and [12] (i) For any $1 \leq i \leq n$, there is a unique algebra automorphism $T_{i}$ (resp. $T_{i}^{-1}$ ) on $U_{h}(G)$ such that

$$
\begin{aligned}
& T_{i}\left(E_{i}\right)=-F_{i} K_{i},\left(\text { resp. } T_{i}^{-1}\left(E_{i}\right)=-K_{i}^{-1} F_{i}\right), \\
& T_{i}\left(F_{i}\right)=-K_{i}^{-1} E_{i},\left(\text { resp. } T_{i}^{-1}\left(F_{i}\right)=-E_{i} K_{i}\right), \\
& T_{i}\left(E_{j}\right)=\sum_{r+s=-a_{i j}}(-1)^{r} q_{i}^{-s} E_{i}^{(r)} E_{j} E_{i}^{(s)}=0,
\end{aligned}
$$

(resp. $\left.T_{i}^{-1}\left(E_{j}\right)=\sum_{r+s=-a_{i j}}(-1)^{r} q_{i}^{-s} E_{i}^{(s)} E_{j} E_{i}^{(r)}=0\right)(i \neq j)$,

$$
T_{i}\left(F_{j}\right)=\sum_{r+s=-a_{i j}}(-1)^{r} q_{i}^{s} F_{i}^{(s)} E_{j} E_{i}^{(r)}=0
$$

(resp. $\left.T_{i}^{-1}\left(F_{j}\right)=\sum_{r+s=-a_{i j}}(-1)^{r} q_{i}^{s} E_{i}^{(r)} E_{j} E_{i}^{(s)}=0\right)(i \neq j)$

$$
T_{i}\left(H_{\lambda}\right)=H_{s_{i}(\lambda)}\left(\text { resp. } T_{i}^{-1}\left(H_{\lambda}\right)=H_{s_{i}(\lambda)}\right)(\lambda \in \mathscr{E})
$$

(ii) $T_{i}$ 's satisfy the braid relations:

$$
\underbrace{T_{i} T_{j} T_{i} \cdots}_{m_{i j}}=\underbrace{T_{j} T_{i} T_{j}}_{m_{i j}} \cdots \quad(1 \leq i \neq j \leq n)
$$

where $m_{i j}=2+4\left(\alpha_{i}, \alpha_{j}\right)^{2} /\left(\alpha_{i}, \alpha_{i}\right)\left(\alpha_{j}, \alpha_{j}\right)$. In particular, for any $w \in \mathscr{W}$, there is a unique element $T_{w}$ such that $T_{w}=T_{i_{1}} \cdots T_{i_{r}}$ for any reduced expression $w=s_{i_{1}} \cdots s_{i_{r}}$.

7.2. Let $\Phi(\subset \mathscr{E})$ be the set of the roots of $\boldsymbol{G}$ and $\Phi_{+}$the set of the positive roots with respect to $\Pi$. Put $N_{-}=N_{+}^{\circ}$. Lusztig proved:

Proposition 7.2.1. ([11] and [12]) (i) If $w \in \mathscr{W}$ and $\alpha_{i} \in \Pi$ satisfies $w\left(\alpha_{i}\right) \in \Phi_{+}$, then $T_{w}\left(E_{i}\right) \in N_{+}$and $T_{w}\left(F_{i}\right) \in N_{-}$.

(ii) If $w \in \mathscr{W}$ and $\alpha_{i}, \alpha_{j} \in \Pi$ satisfies $w\left(\alpha_{i}\right)=\alpha_{j}$, then $T_{w}\left(E_{i}\right)=E_{j}$ and $T_{w}\left(F_{i}\right)=F_{j}$. 


\section{§8. Commutation Relations for Root Vectors of $\mathscr{N}_{+}$(type $\left.F_{4}\right)$}

8.1. First, in the subsections $8.1-3$, we treat $N_{+}\left(\subset U_{h}\left(G\left(F_{4}\right)\right)\right)$ associated to the complex simple Lie algebra $\boldsymbol{G}\left(F_{4}\right)$ of type $F_{4}$. We denote this $N_{+}$ by $\ddot{N}_{+}$. In $\S 8$, the symbols $\ddot{\Pi}, \ddot{\alpha}_{i}, \ddot{I}_{+}, \ddot{E}_{i}, \cdots$ respectively mean $\Pi, \alpha_{i}, I_{+}$, $E_{i}, \cdots$ defined for the simple Lie algebra $G\left(F_{4}\right)$. So, for example, II is the set of simple roots of $\boldsymbol{G}\left(F_{4}\right)$ in an Euclidean space $\ddot{\mathscr{E}}$. Namely $\ddot{\Pi}=\left\{\ddot{\alpha}_{1}, \ddot{\alpha}_{2}, \ddot{\alpha}_{3}, \ddot{\alpha}_{4}\right\}$ with $\ddot{\alpha}_{1}=\varepsilon_{2}-\varepsilon_{3}, \ddot{\alpha}_{2}=\frac{1}{2}\left(\varepsilon_{1}-\varepsilon_{2}-\varepsilon_{3}-\varepsilon_{4}\right), \ddot{\alpha}_{3}=\varepsilon_{4}, \ddot{\alpha}_{4}=\varepsilon_{3}-\varepsilon_{4}$ where $\varepsilon_{i}(1 \leq i \leq 4)$ is a basis of $\mathscr{E}$ satisfying $\left(\varepsilon_{i}, \varepsilon_{j}\right)=\delta_{i j}$. The Dynkin diagram of $(\ddot{\mathscr{E}}, \ddot{\Pi})$ is given by:

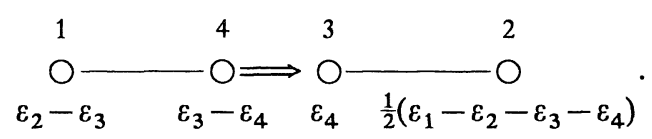

8.2. Let $\ddot{\Phi}_{+}$be the set of positive roots of $\boldsymbol{G}\left(F_{4}\right)$. Put $\ddot{\Phi}_{+, 1}=$ $\left\{\ddot{\beta}=\ddot{\Phi}_{+} \mid \ddot{\beta}=\ddot{\alpha}_{1}+n_{4} \ddot{\alpha}_{4}+n_{3} \ddot{\alpha}_{3}+n_{2} \ddot{\alpha}_{2}\right\}$. Then the number of elements $\ddot{\Phi}_{+, 1}$ is equal to 15 . Define $w_{1} \in \mathscr{W}$ by the following reduced expression:

$$
w_{1}=s_{1} s_{2} s_{3} \cdot s_{2} s_{4} \cdot s_{1} s_{3} \cdot s_{2} \cdot s_{3} s_{1} \cdot s_{4} s_{2} \cdot s_{3} s_{2} s_{1}
$$

The following lemma can be verified directly.

Lemma 8.2.2. For $1 \leq t \leq 15$, let $s_{i_{t}}$ be the $t$-th generator in the reduced expression (8.2.1) of $w_{1}$. We put $\ddot{\beta}_{t}=s_{i_{1}} \cdots s_{i_{t-1}}\left(\ddot{\alpha}_{i_{t}}\right)$. Then $\ddot{\Phi}_{+, 1}=\left\{\ddot{\beta}_{t}\right.$ $(1 \leq t \leq 15)\}$.

For $\ddot{\beta}_{t}=s_{i_{1}} \cdots s_{i_{t-1}}\left(\ddot{\alpha}_{i_{t}}\right) \in \ddot{\Phi}_{+, 1}$, put $e_{\ddot{\beta}_{t}}=T_{i_{1}} \cdots T_{i_{t-1}}\left(\ddot{E}_{i_{t}}\right)$. By Proposition 7.2.1, we see that $e_{\ddot{\beta}_{t}} \in N_{+}$.

8.3. By using Proposition 7.2.1, and reducing to rank 2 cases, we can obtain the following identities. Here we put $e_{a b c d}=e_{a \ddot{\alpha}_{1}+b \ddot{\alpha}_{4}+c \ddot{\alpha ̆}_{3}+d \ddot{\alpha}_{2}}$. See also [12].

Lemma 8.3.1. The following identities hold. 


$$
\begin{gathered}
-\left[e_{1110}, \ddot{E}_{2}\right]_{q^{-1}}=e_{1111},\left[e_{1111}, e_{1120}\right]=0, \\
-\left[e_{1120}, \ddot{E}_{2}\right]_{q^{-2}}=e_{1121}, \\
-\left[e_{1111}, \ddot{E}_{4}\right]=0,-\left[e_{1111}, \ddot{E}_{3}\right]_{q^{-1}}=e_{1121}, \\
-\left[e_{1220}, \ddot{E}_{2}\right]_{q^{-2}}=e_{1221},\left[e_{1121}, e_{1220}\right]=0, \\
-\left[e_{1121}, \ddot{E}_{4}\right]_{q^{-2}}=e_{1221},\left[e_{1121}, \ddot{E}_{3}\right]_{q}=0, \\
{\left[e_{1221}, \ddot{E}_{4}\right]_{q^{2}}=0,-\left[e_{1221}, \ddot{E}_{3}\right]_{q}=e_{1231},} \\
\quad\left[e_{1231}, \ddot{E}_{4}\right]=0,-\left[e_{1231}, \ddot{E}_{3}\right]_{q}=0 .
\end{gathered}
$$

8.4. For type $F_{4}$, we use the following fact.

Lemma 8.4.1. Let $\mathscr{N}_{+}$be the R-algebra defined for the distinguished triple system $(\mathscr{E}, \Pi, p)$ of type $F_{4}($ see $\S 4) . \quad$ Let $v=n_{1} \alpha_{1}+n_{4} \alpha_{4}+n_{3} \alpha_{3}+n_{2} \alpha_{2} \in P_{+}$ be such that $n_{2}=0$ or 1 . Then there exists an $R$-module isomorphism $j_{v}: N_{+, v} \rightarrow \mathscr{N}_{+, v}$ such that $j_{v}\left(\ddot{E}_{i_{1}} \cdots \ddot{E}_{i_{u}}\right)=E_{i_{1}} \cdots E_{i_{u}}$ for any monomial $\ddot{E}_{i_{1}} \cdots \ddot{E}_{i_{u}}$ $\left(\alpha_{i_{1}}+\cdots+\alpha_{i_{u}}=v\right)$ in $\ddot{N}_{+, v}$.

Proof. By Theorem 2.10.1, $\ddot{I}_{+}=\left(y_{i j}(i \neq j)\right)$ where $y_{i j}(i \neq j)$ are elements given in (2.10.2). Let $\mathscr{I}_{+}$be the ideal of $\tilde{N}_{+}$defined in Definition 4.2.1 for type $F_{4}$. Then, for $v \in P_{+}, \ddot{N}_{+, v}=\tilde{N}_{+, v} /\left(\ddot{I}_{+} \cap \tilde{N}_{+, v}\right), \quad \mathscr{N}_{+, v}=$ $\tilde{N}_{+, v} /\left(\mathscr{I}_{+} \cap \tilde{N}_{+, v}\right)$. The lemma now follows by observing that $\ddot{I}_{+} \cap \tilde{N}_{+, v}=$ $\mathscr{I}_{+} \cap \tilde{N}_{+, v}$ if $v=n_{1} \alpha_{1}+n_{4} \alpha_{4}+n_{3} \alpha_{3}+n_{2} \alpha_{2} \in P_{+}$with $n_{2}=0$ or 1 .

8.5. By Lemma 8.3.1, we can easily show:

Lemma 8.5.1. Let $\alpha=a \alpha_{1}+b \alpha_{4}+c \alpha_{3}+d \alpha_{2} \in \Phi_{+, 1}^{\mathrm{red}} \backslash\left\{\alpha_{1}+2 \alpha_{4}+3 \alpha_{3}+2 \alpha_{2}\right\}$. Then we have

$$
j_{v}\left(e_{a \ddot{\alpha}_{1}+b \ddot{\alpha}_{4}+c \ddot{\alpha}_{3}+d \ddot{\alpha}_{2}}\right)=-(-1)^{a+b+c+d} E_{a \alpha_{1}+b \alpha_{4}+c \alpha_{3}+d \alpha_{2}} .
$$

8.6. Proof of Lemma 5.2.1 (i) for $\Phi_{+}^{\text {red }}$ of type $F_{4}$. Here we put $E_{a b c d}=E_{a \alpha_{1}+b \alpha_{4}+c \alpha_{3}+d \alpha_{2}}$. By Lemma 8.3.1, Lemma 8.4.1 and Lemma 5.2.1 (i) for type $B_{3}$ and $C_{3}$ (see $\S 6$ ), it is enough to show:

$$
\left[E_{\alpha}, E_{2}\right]_{q^{-\left(\alpha, \alpha_{2}\right)}}=0 \text { for } E_{\alpha}=E_{1111}, E_{1121}, E_{1221}, E_{1232} \text {, }
$$

and 


$$
\left[E_{1232}, E_{4}\right]=\left[E_{1232}, E_{3}\right]=0
$$

Since $E_{2}^{2}=0$ and $E_{\alpha} \in R\left[E_{\alpha-\alpha_{2}}, E_{2}\right]_{q-\left(\alpha-\alpha_{2}, \alpha_{2}\right)}$, the formulas (8.6.1) follow from (4.4.3).

Since $E_{1232}=\left(q^{2}+1+q^{-2}\right)^{-1}\left[E_{1231}, E_{2}\right]_{q^{-3}}$, by Lemma 8.3.1 and Lemma 8.4.1, we have $\left[E_{1232}, E_{4}\right]=0$. By Lemma 8.3.1, Lemma 8.4.1, (8.6.1) and (4.4.5), we have:

$$
\begin{aligned}
0 & =\left[\left[\left[E_{1221}, E_{2}\right]_{q^{-2}}, E_{3}\right]_{q^{-2}}, E_{3}\right] \\
& =\left(q+q^{2}\right)\left\{q^{-1} E_{1231} E_{0011}+q^{-3} E_{0011} E_{1231}\right\} \\
& =\left(q+q^{-1}\right)\left[E_{1231}, E_{0011}\right]_{q^{-2}}=\left(q+q^{-1}\right)\left[E_{1232}, E_{3}\right] .
\end{aligned}
$$

8.7. Proof of Lemma 5.2.1 (iii) for type $F_{4}$. By Lemma 5.2 .1 (iii) for type $C_{3}$ (see $\S 6$ ), it is enough to show:

$$
E_{\alpha}^{2}=0 \text { if } \alpha \in \Phi_{+, 1}^{\mathrm{red}} \text { and }(\alpha, \alpha)=0
$$

We show (8.7.1) by the induction on $h t(\alpha)$. Since $E_{2}^{2}=0$ and $E_{1111}=\left[E_{1110}, E_{2}\right]_{q^{-1}}, \quad\left[E_{1111}, E_{2}\right]_{q^{-1}}=0$. Hence, by Lemma 8.3 .1 and Lemma 8.4.1,

$$
0=\left[\left[E_{1110}, E_{2}\right]_{q^{-1}}, E_{1111}\right]_{q^{2}}=\left(1+q^{2}\right) E_{1111}^{2}
$$

If $h t(\alpha)>4, E_{\alpha}=\left[E_{\beta}, E_{i}\right]_{q^{-\left(\alpha, \alpha_{i}\right)}}$ for some $i \in\{3,4\}$ and $\beta \in \Phi_{+, 1}^{\text {red }}$ such that $(\beta, \beta)=0$. In this case, $\left[E_{\alpha}, E_{i}\right]_{q^{-\left(\alpha, \alpha_{i}\right)}}=0$ by Lemma 8.3.1 and Lemma 8.4.1, and $\left[E_{\beta}, E_{\alpha}\right]_{q^{-(\alpha, \beta)}}=0$ since $E_{\beta}^{2}=0$ and (4.4.3). Hence we have:

$$
\begin{aligned}
0 & =\left[\left[E_{\beta}, E_{i}\right]_{q^{-\left(\beta, \alpha_{i}\right)},} E_{\alpha}\right]_{q^{\left\{-(\alpha, \beta)+\left(\alpha, \alpha_{i}\right)\right\}}} \\
& =\left(1+q^{\left\{-(\alpha, \beta)+\left(\alpha, \alpha_{i}\right)\right\}}\right) E_{\alpha}^{2} .
\end{aligned}
$$

8.8. Proof of Lemma 5.2.1 (ii) for type $F_{4}$. By Lemma 5.2 .1 (ii) for type $B_{3}$ and $C_{3}$ (see $\S 6$ ), it is enough to show:

$$
\left[E_{\alpha}, E_{\beta}\right]_{q^{-(\alpha, \beta)}}=0 \text { if } \alpha, \beta \in \Phi_{+, 1}^{\mathrm{red}} \text {. }
$$


By Lemma 8.3.1, Lemma 8.4.1 and (8.7.1), (4.4.3), we can easily show:

$$
\left[E_{\alpha}, E_{\beta}\right]=0 \text { if } \alpha, \beta \in \Phi_{+, 1}^{\mathrm{red}} \text { and } h t(\beta)=h t(\alpha) \text {, }
$$

and

$$
\left[E_{\alpha}, E_{\beta}\right]_{q^{-(\alpha, \beta)}}=0 \text { if } \alpha, \beta \in \Phi_{+, 1}^{\mathrm{red}} \text { and } h t(\beta)-h t(\alpha)=1
$$

In the case of $h t(\beta)-h t(\alpha) \geq 2$, we can choose the elements $\gamma \in \Phi_{+, 1}^{\text {red }}$ and $\alpha_{i} \in \Pi$ in such a way that $E_{\beta}=X\left[E_{\gamma}, E_{i}\right]_{q-\left(\gamma, \alpha_{i}\right)}$ holds for some $X \in R^{\times}$. By (4.4.2), we see:

$$
\begin{aligned}
& {\left[E_{\alpha},\left[E_{\gamma}, E_{i}\right]_{q}-\left(\gamma, \alpha_{i}\right)\right]_{q}-\left(\alpha, \gamma+\alpha_{i}\right) } \\
&= {\left.\left[\left[E_{\alpha}, E_{\gamma}\right]_{q}^{-(\alpha, \gamma)}, E_{i}\right]\right]_{q}^{-\left(\alpha+\gamma, \alpha_{i}\right)} } \\
&+(-1)^{p(\alpha) p(\gamma)} q^{-(\alpha, \gamma)} E_{\gamma}\left[E_{\alpha}, E_{i}\right]_{q}^{-\left(\alpha, \alpha_{i}\right)} \\
&-(-1)^{p(\gamma) p\left(\alpha_{i}\right)} q^{-\left(\gamma, \alpha_{i}\right)}\left[E_{\alpha}, E_{i}\right]_{q}^{-\left(\alpha, \alpha_{i}\right)} E_{\gamma} .
\end{aligned}
$$

Since $\alpha<\gamma<\beta$, we finish the proof using part (i) of Lemma 5.2.1.

\section{§9. Commutation Relations for Root Vectors of $\mathscr{N}_{+}$(type $G_{3}$ )}

9.1. Let $\left(\mathscr{E}, \Pi=\left\{\alpha_{1}, \alpha_{2}, \alpha_{3}\right\}, p\right)$ be the distinguished triple system of type $G_{3}$ (see $\S 3$ ). Let $\mathscr{U}_{h}=\mathscr{U}_{h}\left(\mathscr{G}\left(G_{3}\right)\right)$ be an $h$-adic $R$-algebra with generators $E_{i}, F_{i}(1 \leq i \leq 3), H \in \mathscr{H}$ and relations:

$$
\left[H_{1}, H_{2}\right]=0\left(H_{1}, H_{2} \in \mathscr{H}\right)
$$

$$
\begin{aligned}
& {\left[H, E_{i}\right]=\alpha_{i}(H) E_{i},\left[H, F_{i}\right]=-\alpha_{i}(H) F_{i}(H \in \mathscr{H}),} \\
& E_{i} F_{j}-(-1)^{p\left(\alpha_{i}\right) p\left(\alpha_{j}\right)} F_{j} E_{i}=\delta_{i j} \frac{\operatorname{sh}\left(h H_{\alpha_{i}}\right)}{\operatorname{sh}\left(h d_{i}\right)}
\end{aligned}
$$

$$
\begin{aligned}
& E_{1}^{2}=0, \\
& \sum_{v=0}^{1+\left|a_{i j}\right|}(-1)^{v}\left[\begin{array}{c}
1+\left|a_{i j}\right| \\
v
\end{array}\right]_{q^{d_{i}}} E_{i}^{1+\left|a_{i j}\right|-v} E_{j} E_{i}^{v}=0 \text { for } i \neq j \text { and }
\end{aligned}
$$




$$
p\left(\alpha_{i}\right)=0
$$

(9.1.5) $\quad F_{1}^{2}=0$,

$$
\begin{aligned}
& \sum_{v=0}^{1+\left|a_{i j}\right|}(-1)^{v}\left[\begin{array}{c}
1+\left|a_{i j}\right| \\
v
\end{array}\right]_{q} d_{i} F_{i}^{1+\left|a_{i j}\right|-v} F_{j} F_{i}^{v}=0 \text { for } i \neq j \text { and } \\
& p\left(x_{i}\right)=0 .
\end{aligned}
$$

Let $\mathcal{N}_{+}$be the $R$-algebra with generators $E_{1}, E_{2}, E_{3}$ which was defined in $\S 4$ for the distinguished triple system $(\mathscr{E}, \Pi, p)$ of type $G_{3}$. Then it is obvious that there exists an $R$-algebra map $i_{+}: \mathscr{N}_{+} \rightarrow \mathscr{U}_{h}$ (resp. $i_{-}: \mathscr{N}_{+} \rightarrow \mathscr{U}_{h}$ ) such that $i_{+}\left(E_{i}\right)=E_{i}$ (resp. $\left.i_{+}\left(E_{i}\right)=F_{i}\right)(i=1,2,3)$. Let $\mathscr{N}_{-}=i_{-}\left(\mathscr{N}_{+}\right)$and $\mathscr{N}_{-, \alpha}=i_{-}\left(\mathscr{N}_{+, \alpha}\right)\left(\alpha \in P_{+}\right)$.

Put $U_{h}\left(\boldsymbol{G}\left(G_{2}\right)\right)=U_{h}\left(\boldsymbol{C} \alpha_{3} \oplus \boldsymbol{C} \alpha_{2},\left\{\alpha_{3}, \alpha_{2}\right\}\right)$ (see 7.1).

Similarly to Theorem 1 (iii)-(iv) of [19], we have:

Lemma 9.1.6.(The triangular decomposition of $\left.\mathscr{U}_{h}=\left(\mathscr{G}\left(G_{3}\right)\right)\right)$

(i) The maps $i_{+}$and $i_{-}$are injective. As h-adic topological $R$-modules,

$$
\mathscr{U}_{h} \simeq \mathscr{N}_{+} \hat{\otimes} \subseteq\left[\mathscr{H}^{R}\right] \hat{\otimes} \mathscr{N}_{-} .
$$

(ii) There exists an injective h-adic topological algebra map j: $U_{h}\left(G\left(G_{2}\right)\right) \rightarrow$ $\mathscr{U}_{h}$ such that $j\left(E_{i}\right)=E_{i}, j\left(F_{i}\right)=F_{i}(i=3,2)$ and $j\left(H_{\lambda}\right)=H_{\lambda}(\lambda \in \ddot{E})$.

We omit the proof.

9.2. We shall extend the braid group action on $U_{h}\left(\left(G\left(G_{2}\right)\right)\right.$ in $\S 7$ to the one on $U_{h}\left(\left(\mathscr{G}\left(G_{3}\right)\right)\left(\supset \mathscr{U}_{h}\left(G\left(G_{2}\right)\right)\right)\right.$. By direct computations, we can show the following lemma. We omit the proof.

Lemma 9.2.1. Let $T_{i}, T_{i}^{-1} \in \operatorname{Aut}\left(U_{h}\left(\left(\boldsymbol{G}\left(G_{2}\right)\right)\right)(i=3,2)\right.$ be of Proposition 7.2.1. Then $T_{i}, T_{i}^{-1}(i=3,2)$ can be extended to automorphisms of $\mathscr{U}_{h}\left(\mathscr{G}\left(G_{3}\right)\right)$ such that

$$
T_{3}\left(E_{1}\right)=-E_{3} E_{1}+q^{-1} E_{1} E_{3}, T_{2}\left(E_{1}\right)=E_{1}
$$




$$
\begin{aligned}
& T_{3}^{-1}\left(E_{1}\right)=-E_{1} E_{3}+q^{-1} E_{3} E_{1}, T_{2}^{-1}\left(E_{1}\right)=E_{1}, \\
& T_{3}\left(F_{1}\right)=-q F_{3} F_{1}+F_{1} F_{3}, T_{2}\left(F_{1}\right)=F_{1}, \\
& T_{3}^{-1}\left(F_{1}\right)=-q F_{1} F_{3}+F_{3} F_{1}, T_{2}^{-1}\left(F_{1}\right)=F_{1}, \\
& T_{i}\left(H_{\lambda}\right)=H_{s_{i}(\lambda)}\left(\operatorname{resp} . T_{i}^{-1}\left(H_{\lambda}\right)=H_{s_{i}(\lambda)}\right)(\lambda \in \mathscr{E})(i=3,2) .
\end{aligned}
$$

\subsection{Put}

$$
\begin{aligned}
& \text { (9.3.1) } e_{110}=T_{3}\left(E_{1}\right), e_{111}=T_{2} T_{3}\left(E_{1}\right), e_{131}=T_{3} T_{2} T_{3}\left(E_{1}\right), \\
& \text { (9.3.2) } \left.e_{121}=\left(q+q^{-1}\right)^{-1} \llbracket e_{111}, E_{3} \rrbracket \text { (see } 4.4 \text { for the notation } \llbracket, \rrbracket\right) . \\
& \text { (9.3.3) } e_{132}=T_{2} T_{3} T_{2} T_{3}\left(E_{1}\right), e_{142}=T_{2} T_{3} T_{2} T_{3} T_{2} T_{3}\left(E_{1}\right) .
\end{aligned}
$$

Lemma 9.3.4. We have:

$$
\llbracket e_{a b c}, E_{i} \rrbracket=0
$$

if $i \in\{3,2\}$ and $a \alpha_{1}+b \alpha_{3}+c \alpha_{2}+\alpha_{i} \notin \Phi_{+}^{\text {red }}$.

Proof. We are in one of the following three cases.

(i) $(a, b, c, i)=(1,1,0,3),(1,1,1,2),(1,3,1,3),(1,4,2,2)$,

(ii) $(a, b, c, i)=(1,2,1,1)$,

(iii) $(a, b, c, i)=(1,4,2,3)$.

(i) In this case, if we write $e_{a b c}=T_{i_{1}} T_{i_{2}} \cdots T_{i_{u}}\left(E_{1}\right)$ as in (9.3.1-3), then, by Proposition 7.1.1 and Proposition 7.2.1, we have:

$$
\left(T_{i_{1}} T_{i_{2}} \cdots T_{i_{u}}\right)^{-1}\left(E_{i}\right)=-\exp \left(-h H_{\gamma}\right) X
$$

where $\gamma=\left(s_{i_{2}} \cdots s_{i_{u}}\right)^{-1}\left(\alpha_{i}\right)$ and $X=\left(T_{i_{2}} \cdots T_{i_{u}}\right)^{-1}\left(F_{i}\right) \in \mathcal{N}_{-, \gamma}$. Hence we have the formula in this case.

(ii) By (4.4.2) and (i), we have:

$$
\begin{aligned}
\llbracket e_{121}, E_{2} \rrbracket & =\left(q+q^{-1}\right)^{-1} \llbracket \llbracket e_{111}, E_{3} \rrbracket, E_{2} \rrbracket \\
& =\left(q+q^{-1}\right)^{-1} \llbracket e_{111}, \llbracket E_{3}, E_{2} \rrbracket \rrbracket=\left(q+q^{-1}\right)^{-1} \llbracket T_{2} T_{3}\left(E_{1}\right),-T_{2}\left(E_{3}\right) \rrbracket
\end{aligned}
$$




$$
=-\left(q+q^{-1}\right)^{-1} T_{2}\left(\llbracket e_{110}, E_{3} \rrbracket\right)=0 .
$$

(iii) By Proposition 7.2.1 and (i), we have:

$$
\begin{aligned}
\llbracket e_{142}, E_{3} \rrbracket & =\llbracket T_{2} T_{3} T_{2} T_{3} T_{2} T_{3}\left(E_{1}\right), T_{2} T_{3} T_{2} T_{3} T_{2}\left(E_{3}\right) \rrbracket \\
& =T_{2} T_{3} T_{2} T_{3} T_{2}\left(\llbracket e_{110}, E_{3} \rrbracket\right)=0
\end{aligned}
$$

This completes the proof.

Lemma 9.3.5. We have:

(i) $e_{110}=q^{-1} \llbracket E_{1}, E_{3} \rrbracket$.

(ii) $e_{142}=q^{-1} \llbracket e_{132}, E_{3} \rrbracket$.

(iii)

$$
\begin{aligned}
& e_{111}=q^{-3} \llbracket e_{110}, E_{2} \rrbracket, e_{131}=q^{-2} \llbracket e_{121}, E_{3} \rrbracket \\
& e_{132}=q^{-3} \llbracket e_{131}, E_{2} \rrbracket .
\end{aligned}
$$

In particular, $e_{a b c} \in \mathscr{N}_{+}$.

Proof. (i) Clear.

(ii) $e_{142}=T_{2} T_{3} T_{2} T_{3} T_{2} T_{3}\left(E_{1}\right)=T_{2} T_{3} T_{2} T_{3} T_{2}\left(e_{110}\right)$

$$
=q^{-1} \llbracket T_{2} T_{3} T_{2} T_{3}\left(E_{1}\right), T_{2} T_{3} T_{2} T_{3} T_{2}\left(E_{3}\right) \rrbracket .
$$

By Proposition 7.2.1, $T_{2} T_{3} T_{2} T_{3} T_{2}\left(E_{3}\right)=E_{3}$. Hence

$$
e_{142}=q^{-1} \llbracket e_{132}, E_{3} \rrbracket
$$

(iii) The formulas (9.3.6) can be verified by direct computations. We sketch the proof. We write $e_{1 b c}=T_{i_{1}} \cdots T_{i_{u-1}} T_{i_{u}}\left(E_{1}\right)$ as in (9.3.1-3). Then

$$
e_{1 b c}=q^{-1} T_{i_{1}} \cdots T_{i_{u-1}}\left(\llbracket E_{1}, E_{3} \rrbracket\right)=q^{-1} \llbracket e_{1 y z}, T_{i_{1}} \cdots T_{i_{u-1}}\left(E_{3}\right) \rrbracket .
$$

Here, if $(b, c)=(1,1) \quad$ (resp. $(3,1),(3,2))$, then $(y, z)=(0,0) \quad($ resp. $(1,0)$, $(1,1))$. By Proposition 7.2 .1 (ii), $T_{i_{1}} \cdots T_{i_{u-1}}\left(E_{3}\right) \in \mathscr{N}_{+,(b-y) \alpha_{3}+(c-z) \alpha_{2}}$. In fact, by direct computations, we can show that $T_{2}\left(E_{3}\right)=q^{-3} \llbracket E_{3}, E_{2} \rrbracket$ (resp. 
$T_{3} T_{2}\left(E_{3}\right)=q^{-4}\left(q+q^{-1}\right)^{-1} \llbracket \llbracket E_{2}, E_{3} \rrbracket, E_{3} \rrbracket, T_{2} T_{3} T_{2}\left(E_{3}\right)=q^{-4}\left(q+q^{-1}\right)^{-1}$ $\llbracket \llbracket E_{3}, E_{2} \rrbracket, E_{2} \rrbracket$ if $(b, c)=(1,1)$ (resp. $\left.(3,1),(3,2)\right)$. By the formulas in Lemma 9.3.1 and the formulas which we have already shown in this lemma, and by using the formula (4.4.2) repeatedly, we have the formulas (iii). For example,

$$
\begin{aligned}
e_{111} & =T_{2} T_{3}\left(E_{1}\right)=T_{2}\left(e_{110}\right)=q^{-1} \llbracket E_{1}, T_{2}\left(E_{3}\right) \rrbracket \\
& =q^{-4} \llbracket \llbracket E_{1}, E_{3} \rrbracket, E_{2} \rrbracket=q^{-3} \llbracket e_{110}, E_{2} \rrbracket .
\end{aligned}
$$

This proves the first formula. The second (resp. third) formula can be proved similarly using the first one (resp. the first and the second ones).

Lemma 9.3.7. We have:

(i) $e_{1 b c}^{2}=0$ if $(b, c) \neq(2,1)$.

(ii) $\llbracket e_{1 b c}, e_{1 y z} \rrbracket=0$ if $b+c-y-z=1$.

Proof. (i) This is obvious from (9.3.1) and (9.3.3).

(ii) If $(b, c) \neq(3,1)$, then, by Lemma 9.3.5, $e_{1 b c} \in R \llbracket e_{1 y z} . E_{i} \rrbracket$ for some $i \in\{3,2\}$. By (i), $e_{1 y z}^{2}=0$. Hence, by (4.4.3), we have (ii). If $(b, c)=(3,1)$, then $(y, z)=(2,1)$.

Since $\llbracket e_{111}, e_{121} \rrbracket=0$, we have

$$
\begin{aligned}
0= & \llbracket \llbracket \llbracket\left[e_{111}, e_{121} \rrbracket, E_{3} \rrbracket, E_{3} \rrbracket\right. \\
= & \left(1+q^{-2}\right)\left\{e_{121}\left(q^{2} e_{131}\right)-(-1) q^{4}\left(q^{2} e_{131}\right) e_{121}\right\} \\
& +\left(q^{2} e_{131}\right) e_{121}-(-1) q^{2} e_{121}\left(q^{2} e_{131}\right) \\
= & q^{2}\left(q^{-2}+1+q^{2}\right) \llbracket e_{121}, e_{131} \rrbracket
\end{aligned}
$$

by (4.4.5). Hence we get (ii).

For $\alpha=a \alpha_{1}+b \alpha_{3}+c \alpha_{2} \in \Phi_{+}^{\text {red }}$, put $E_{a b c}=E_{a \alpha_{1}+b \alpha_{3}+c \alpha_{2}}$. The next lemma easily follows from Lemma 9.3.5.

Lemma 9.3.8. We have: 


$$
\begin{aligned}
& E_{110}=q e_{110}, E_{111}=q^{4} e_{111}, E_{121}=q^{4} e_{121}, \\
& E_{131}=q^{6} e_{131}, E_{132}=q^{9} e_{132}, E_{142}=q^{10} e_{142}
\end{aligned}
$$

By Lemma 9.3.7 and Lemma 9.3.8, and an argument similar to that in 8.8, we have:

Lemma 9.3.9. Let $\alpha, \beta \in \Phi_{+, 1}^{\mathrm{red}}$ be such that $\alpha<\beta$. Then we have:

$$
\left[E_{\alpha}, E_{\beta}\right]_{q^{-(\alpha, \beta)}}=\sum_{\gamma_{1}, \cdots, \gamma_{u} \in \Phi_{+, 1}^{\mathrm{red}}(\alpha<\beta)} c_{\gamma_{1}, \cdots, \gamma_{u}} E_{\gamma_{1}} \cdots E_{\gamma_{u}}
$$

for some $c_{\gamma_{1}, \cdots, \gamma_{u}} \in R$.

9.4. By the definition of $\mathscr{U}_{h}$ (see 9.1), we can easily see that there is a $C$-algebra isomorphism $r: \mathscr{U}_{h} \rightarrow \mathscr{U}_{h}$ such that

$$
r\left(E_{i}\right)=E_{i}, r\left(F_{i}\right)=F_{i}, r(H)=H(H \in \mathscr{H}), r(h)=-h
$$

Put

$$
\begin{aligned}
& e_{010}=E_{3}, e_{011}=T_{2}\left(E_{3}\right), e_{032}=T_{2} T_{3}\left(E_{2}\right) \\
& e_{021}=T_{2} T_{3} T_{2}\left(E_{3}\right), e_{031}=T_{2} T_{3} T_{2} T_{3}\left(E_{2}\right), e_{001}=E_{2}
\end{aligned}
$$

By Proposition 7.2.1, we see that the above elements belong to $\mathcal{N}_{+}$. By direct computations, we can get commutation relations for the above elements. For example, such commutation relations are found in Section 5 in [12]. From them, we have:

Lemma 9.4.1. We have:

(i) $E_{010}=E_{3}, E_{011}=-r\left(e_{011}\right), E_{032}=-r\left(e_{032}\right)$,

$$
E_{021}=r\left(e_{021}\right), E_{031}=-r\left(e_{031}\right), E_{001}=E_{2} .
$$

(ii) The $q$-root vectors $\left\{E_{010}, E_{011}, E_{032} E_{021}, E_{031}, E_{001}\right\}$ satisfy the commutator relations in Lemma 5.2 .1 (i)-(ii).

9.5. By lemmas in 9.3-4, we can prove Lemma 5.2.1 and Remark 5.2.2 
for $\mathscr{N}_{+}$of type $G_{3}$.

\section{§10. Main Results}

10.1. Let $\left(\mathscr{E}, \Pi=\left\{\alpha_{1}, \cdots, \alpha_{n}\right\}, p\right)$ be the triple system satisfying the assumption in 3.1 .

Lemma 10.1.1. Let $\alpha \in \Phi_{+}^{\mathrm{red}}$. Then, in the $h$-adic topological $R^{\prime}$-bialgebra, $\mathscr{U}_{\sqrt{h}}^{\prime} b_{+}^{\sigma}$, we have:

(10.1.2) $\Delta^{\prime}\left(E_{\alpha}\right)-\left\{E_{\alpha} \otimes 1+\exp \left(\sqrt{h} H_{\alpha}^{\prime}\right) \sigma^{p(\alpha)} \otimes E_{\alpha}\right\}$

$$
\epsilon \sum_{\left.\gamma_{1}, \cdots, \gamma_{u} \in \Phi_{+, g(\alpha)}^{\mathrm{red}}<\alpha\right)} \mathscr{U}_{\sqrt{h}}^{\prime} b_{+}^{\sigma} \otimes E_{\gamma_{1}} \cdots E_{\gamma_{u}} .
$$

Proof. We use the induction with respect, to the order $<$ on $\Phi_{+, g(\alpha)}^{\text {red }}$. Then, by using Definition 5.1.1 and Lemma 5.2.1, we can show that

(10.1.3) $\Delta^{\prime}\left(E_{\alpha}\right)-\left\{E_{\alpha} \otimes 1+\exp \left(\sqrt{h} H_{\alpha}^{\prime}\right) \sigma^{p(\alpha)} \otimes E_{\alpha}\right\}$

$$
\begin{aligned}
& \epsilon \sum_{\left.\gamma_{1}, \cdots, \gamma_{u} \in \Phi_{+, g(\alpha)}^{\text {red }}<\alpha\right)} X_{\alpha-\gamma_{1}-\cdots-\gamma_{u}} \\
& \exp \left(\sqrt{h} H_{\gamma_{1}+\cdots+\gamma_{u}}^{\prime}\right) \sigma^{p\left(\gamma_{1}\right)+\cdots+p\left(\gamma_{u}\right)} \otimes E_{\gamma_{1}} \cdots E_{\gamma_{u}}
\end{aligned}
$$

for some $X_{\alpha-\gamma_{1}-\cdots-\gamma_{u}} \in \mathscr{N}_{+, \alpha-\gamma_{1}-\cdots-\gamma_{u}}$ where $u=c_{\alpha}$ (see 3.2 for the notation $c_{\alpha}$ ).

10.2. Put $\Psi_{n}(t)=\prod_{i=1}^{n} \frac{t^{i}-1}{t-1} \in C[t]$. Let $q=e^{h}$. As an immediate consequence of Lemma 5.2.1 and Lemma 10.1.1, we have:

\section{Lemma 10.2.1.}

$$
\left\langle\prod_{\alpha \in \Phi_{+}^{\text {red }}}^{<} E_{\alpha}^{m_{\alpha}}, \prod_{\alpha \in \Phi_{+}^{\text {red }}}^{<} E_{\alpha}^{n_{\alpha}}\right\rangle
$$




$$
=\prod_{\alpha \in \Phi_{+}^{\text {red }}} \delta_{n_{\alpha}, m_{\alpha}} \Psi_{n_{\alpha}}\left((-1)^{p(\alpha)} q^{(\alpha, \alpha)}\right)\left\langle E_{\alpha}, E_{\alpha}\right\rangle^{n_{\alpha}}
$$

(See 5.3 for the notation $\prod_{\alpha \in \Phi_{+}^{\text {red }}}^{<}$. )

Proof. Note that $\langle$,$\rangle is symmetric. Let \gamma \in \Phi_{+}^{\text {red }}$ be such that $m_{\gamma}+n_{\gamma} \neq 0$ and $m_{\gamma^{\prime}}=n_{\gamma^{\prime}}=0$ for all $\gamma^{\prime}>\gamma$. Assume $m_{\gamma} \geq n_{\gamma}$. By Lemma 5.2.1 and Lemma 10.1.1, we have:

$$
\begin{aligned}
& \left\langle\prod_{\alpha \in \Phi_{+}^{\text {red }}}^{<}, \prod_{\alpha \in \Phi_{+}^{\text {red }}}^{<} E_{\alpha}^{n_{\alpha}}\right\rangle \\
& =\left\langle\left(\prod_{\alpha \in \Phi_{+}^{\text {red }}(<\gamma)}^{<} E_{\alpha}^{m_{\alpha}}\right) E_{\gamma}^{m_{\gamma}-1} \otimes E_{\gamma}, \prod_{\alpha \in \Phi_{+}^{\text {red }}}^{<} \Delta^{\prime}\left(E_{\alpha}\right)^{n_{\alpha}}\right\rangle \\
& =\left\langle\left(\prod_{\alpha \in \Phi_{+}^{\text {red }}(<\gamma)}^{<} E_{\alpha}^{m_{\alpha}}\right) E_{\gamma}^{m_{\gamma}-1} \otimes E_{\gamma},\right. \\
& \left.\left(\prod_{\substack{\text { red } \\
\alpha \in \Phi_{+}(<\gamma)}}^{<}\left(E_{\alpha} \otimes 1\right)^{n_{\alpha}}\right)\left(E_{\gamma} \otimes 1+\exp \left(\sqrt{h} H_{\gamma}^{\prime}\right) \sigma^{p(\gamma)} \otimes E_{\gamma}\right)^{n_{\gamma}}\right\rangle \\
& =\left\langle\left(\prod_{\alpha \in \Phi_{+}^{\text {red }}(<\gamma)}^{<} E_{\alpha}^{m_{\alpha}}\right) E_{\gamma}^{m_{\nu}-1} \otimes E_{\gamma},\right. \\
& \left(\prod_{\alpha \in \Phi_{+}^{\text {red }}(<\gamma)}^{<}\left(E_{\alpha} \otimes 1\right)^{n_{\alpha}}\right) \\
& \left.\cdot\left(\Psi_{n_{\gamma}}\left((-1)^{p(\gamma)} q^{(\gamma, \gamma)}\right) E_{\gamma}^{n_{\gamma}-1} \exp \left(\sqrt{h} H_{\gamma}^{\prime}\right) \sigma^{p(\gamma)} \otimes E_{\gamma}\right)\right\rangle \\
& =\Psi_{n_{\gamma}}\left((-1)^{p(\gamma)} q^{(\gamma, \gamma)}\right) \\
& \left\langle\left(\prod_{\alpha \in \Phi_{+}^{\text {red }}(<\gamma)}^{<} E_{\alpha}^{m_{\alpha}}\right) E_{\gamma}^{m_{\gamma}-1},\left(\prod_{\alpha \in \Phi_{+}^{\text {red }}(<\gamma)}^{<} E_{\alpha}^{n_{\alpha}}\right) E_{\gamma}^{n_{\gamma}-1}\right\rangle
\end{aligned}
$$

where we regared $E_{\gamma}^{-1}$ as 0 . 
Iterating this procedure, we can prove the lemma.

10.3. Here we determine the values $\left\langle E_{\alpha}, E_{\alpha}\right\rangle\left(\alpha \in \Phi_{+}^{\text {red }}\right)$. Define $d_{\alpha} \in Z$ $\left(\alpha \in \Phi_{+}^{\text {red }}\right)$ by

$$
d_{\alpha}=\left\{\begin{array}{cl}
1 & \text { if }(\alpha, \alpha)=0 \\
2 & \text { if } \Phi_{+}^{\text {red }} \text { is of type } G_{3} \text { and } \alpha=\alpha_{1}+2 \alpha_{2}+\alpha_{1} \\
\frac{|(\alpha, \alpha)|}{2} & \text { otherwise }
\end{array}\right.
$$

For $\alpha=c_{1} \alpha_{1}+\cdots+c_{n} \alpha_{n} \in \Phi_{+}^{\text {red }}$, put

$$
b(\alpha)=\left(q^{d_{\alpha}}-q^{-d_{\alpha}}\right)\left\langle E_{\alpha}, E_{\alpha}\right\rangle / \prod_{i=1}^{n}\left(q^{d_{i}}-q^{-d_{i}}\right)^{c_{i}} \in K .
$$

Lemma 10.3.1. For any $\alpha \in \Phi_{+}^{\mathrm{red}}, b(\alpha)$ can be written as

$$
b(\alpha)=(-1)^{a} q^{b}
$$

for some $a, b \in Z$. More precisely, for each type of $\Phi_{+}^{\mathrm{red}}, b(\alpha)\left(\alpha \in \Phi_{+}^{\mathrm{red}}\right)$ are given by:

(i) (Type $\left.A_{N-1}\right)$

$$
b\left(\bar{\varepsilon}_{i}-\bar{\varepsilon}_{j}\right)=\prod_{i<l<j} \bar{d}_{l} q^{\bar{d}_{l}} \quad(i<j) .
$$

(ii) $\left(\right.$ Type $\left.B_{N}\right)$

$$
\begin{aligned}
& b\left(\bar{\varepsilon}_{i}-\bar{\varepsilon}_{j}\right)=\prod_{i<l<j} \bar{d}_{l} q^{\bar{d}_{l}} \quad(i<j), \quad b\left(\bar{\varepsilon}_{i}\right)=\prod_{i<l \leq N} \bar{d}_{l} q^{\bar{d}_{l}}, \\
& b\left(\bar{\varepsilon}_{i}+\bar{\varepsilon}_{j}\right)=(-1)^{p\left(\alpha_{N}\right)} \bar{d}_{N}\left(\prod_{i<l \leq j} \bar{d}_{l} q^{\bar{d}_{l}}\right)\left(\prod_{j<l \leq N} \bar{d}_{l} q^{2 \bar{d}_{l}}\right) \quad(i<j) .
\end{aligned}
$$

(iii) $\left(\right.$ Type $\left.C_{N}\right)$

$$
b\left(\bar{\varepsilon}_{i}-\bar{\varepsilon}_{j}\right)=\prod_{i<l<j} \bar{d}_{l} q^{\bar{d}_{l}} \quad(i<j), \quad b\left(2 \bar{\varepsilon}_{i}\right)=\prod_{i<l \leq N} q^{2 \bar{d}_{l}}\left(p\left(\bar{\varepsilon}_{i}-\bar{\varepsilon}_{N}\right)=0\right),
$$




$$
b\left(\bar{\varepsilon}_{i}+\bar{\varepsilon}_{j}\right)=q^{\bar{d}_{N}}\left(\prod_{i<l \leq j} \bar{d}_{l} q^{\bar{d}_{l}}\right)\left(\prod_{j<l \leq N} \bar{d}_{l} q^{2 \bar{d}_{l}}\right) \quad(i<j)
$$

(iv) (Type $\left.D_{N}\right)$

$$
\begin{aligned}
& b\left(\bar{\varepsilon}_{i}-\bar{\varepsilon}_{j}\right)=\prod_{i<l<j} \bar{d}_{l} q^{\bar{d}_{l}} \quad(i<j), \quad b\left(2 \bar{\varepsilon}_{i}\right)=\bar{d}_{N} \prod_{i<l \leq N} q^{2 \bar{d}_{l}} \quad\left(p\left(\bar{\varepsilon}_{i}-\bar{\varepsilon}_{N}\right)=0\right), \\
& b\left(\bar{\varepsilon}_{i}+\bar{\varepsilon}_{j}\right)=\bar{d}_{N} q^{\bar{d}_{N}}\left(\prod_{i<l \leq j} \bar{d}_{l} q^{\bar{d}_{l}}\right)\left(\prod_{j<l \leq N-1} \bar{d}_{l} q^{2 \bar{d}_{l}}\right) \quad(i<j) .
\end{aligned}
$$

(v) (Type $\left.F_{4}\right)$ Here $b_{a b c d}$ denotes $b\left(a \alpha_{1}+b \alpha_{4}+c \alpha_{3}+d \alpha_{2}\right)$.

$$
\begin{aligned}
& b_{1000}=1, b_{1100}=-q^{-2}, b_{1110}=q^{-4}, b_{1120}=-q^{-4} \\
& b_{1111}=-q^{-5}, b_{1220}=q^{-6}, b_{1121}=q^{-6}, b_{1221}=-q^{-8} \\
& b_{1231}=q^{-9}, b_{1232}=-q^{-12} \\
& b_{0001}=1, b_{0011}=-q^{-1}, b_{0111}=q^{-3}, b_{0121}=-q^{-4} \\
& b_{0010}=1, b_{0110}=-q^{-2}, b_{0120}=q^{-2}, b_{0100}=1
\end{aligned}
$$

(vi) (Type $\left.G_{3}\right)$ Here $b_{a b c}$ denotes $b\left(a \alpha_{1}+b \alpha_{3}+c \alpha_{2}\right)$.

$$
\begin{aligned}
& b_{100}=1, b_{110}=q, b_{111}=q^{4}, b_{121}=q^{6}, \\
& b_{131}=q^{6}, b_{132}=q^{9}, b_{142}=q^{10}, \\
& b_{001}=1, b_{011}=q^{3}, b_{021}=q^{4}, b_{032}=q^{6}, \\
& b_{031}=q^{3}, b_{010}=1 .
\end{aligned}
$$

Proof. Here we sketch how to caluculate $\left\langle E_{\alpha}, E_{\alpha}\right\rangle \quad\left(\alpha \in \Phi_{+}^{\text {red }}\right)$. Put $L_{\alpha}=\exp \left(\sqrt{h} H_{\alpha}^{\prime}\right) \sigma^{p(\alpha)}$ for $\alpha \in \Phi_{+}^{\text {red. }}$. We are in one of the cases (1) $c_{\alpha}=1$ and (2) $c_{\alpha}=2$. Firstly assume that we are in case (1). Suppose $h t(\alpha) \geq 2$ and $\alpha \in \Phi_{+, i}^{\text {red. }}$ In this case, there exists $\alpha_{j} \in \Pi$ such that $\alpha-\alpha_{j} \in \Phi_{+, i}^{\text {red }} . \quad$ Let $r \in Z_{+}$ be such that $\alpha-u \alpha_{j} \in \Phi_{+, i}^{\text {red }}(0 \leq u \leq r)$ and $\alpha-(r+1) \alpha_{j} \notin \Phi_{+, i}^{\text {red }}$.

Put $\beta=\alpha-\alpha_{j}$ and $\gamma=\alpha-r \alpha_{j}$. By the definition of $E_{\alpha}$ (see Definition 
5.1.1), $E_{\beta}=y \llbracket \cdots \llbracket \llbracket\left[E_{\gamma}, E_{j} \rrbracket, E_{j} \rrbracket \cdots, E_{j} \rrbracket\right.$ and $E_{\beta}=x \llbracket\left[E_{\gamma}, E_{j} \rrbracket\right.$ for some $x, y \in R^{\times}$. By (10.1.3), we have:

$$
\begin{aligned}
\left\langle E_{\alpha}, E_{\alpha}\right\rangle= & x\left\langle E_{\beta} \otimes E_{j}-(-1)^{p(\beta) p\left(\alpha_{j}\right)} q^{-\left(\beta, \alpha_{j}\right)} E_{j} \otimes E_{\beta}, \Delta^{\prime}\left(E_{\alpha}\right)\right\rangle \\
= & x^{2} y\left\langle E_{\beta} \otimes E_{j}-(-1)^{p(\beta) p\left(\alpha_{j}\right)} q^{-\left(\beta, \alpha_{j}\right)} E_{j} \otimes E_{\beta},\right. \\
& \llbracket\left[\cdots \cdots \llbracket E_{\gamma} \otimes 1+L_{\gamma} \otimes E_{\gamma}, \Delta^{\prime}\left(E_{j}\right) \rrbracket, \Delta^{\prime}\left(E_{j}\right) \rrbracket, \cdots, \Delta^{\prime}\left(E_{j}\right) \rrbracket\right\rangle .
\end{aligned}
$$

By direct computations, we see that this equals

$$
\begin{aligned}
& x^{2}\left\langle-(-1)^{p(\beta) p\left(\alpha_{j}\right)} q^{-\left(\beta, \alpha_{j}\right)} E_{j} \otimes E_{\beta},\right. \\
& (-1)^{p(\gamma) p\left(\alpha_{j}\right)} q^{\left(\gamma, \alpha_{j}\right)}\left\{1-(-1)^{(r-1) p\left(\alpha_{j}\right)} q^{-\left(2 \gamma+(r-1) \alpha_{j}, \alpha_{j}\right)}\right\} \\
& \left.\frac{1-(-1)^{r p\left(\alpha_{j}\right)} q^{r\left(\alpha_{j}, \alpha_{j}\right)}}{1-(-1)^{p\left(\alpha_{j}\right)} q^{\left(\alpha_{j}, \alpha_{j}\right)}} E_{j} L_{\beta} \otimes E_{\beta}\right\rangle \\
= & -x^{2}(-1)^{(r-1) p\left(\alpha_{j}\right)} q^{-(r-1)\left(\alpha_{j}, \alpha_{j}\right)} \\
& \left\{1-(-1)^{(r-1) p\left(\alpha_{j}\right)} q^{-\left(2 \gamma+(r-1) \alpha_{j}, \alpha_{j}\right)}\right\} \frac{1-(-1)^{r p\left(\alpha_{j}\right)} q^{r\left(\alpha_{j}, \alpha_{j}\right)}}{1-(-1)^{p\left(\alpha_{j}\right)} q^{\left(\alpha_{j}, \alpha_{j}\right)}}\left\langle E_{\beta}, E_{\beta}\right\rangle .
\end{aligned}
$$

Hence we can calculate $\left\langle E_{\alpha}, E_{\alpha}\right\rangle$ by the induction on $h t(\alpha)$.

Next assume that we are in case (2). Suppose $\alpha \in \Phi_{+}^{\text {red. }}$. By the definition of $E_{\alpha}$ (see Definition 5.1.1), there exist $\beta, \gamma \in \Phi_{+}^{\text {red }}$ such that $\alpha=\beta+\gamma$, $h t(\gamma)-h t(\beta) \leq 1$ and $E_{\alpha}=z \llbracket E_{\beta}, E_{\gamma} \rrbracket$ for some $z \in R^{\times}$. If $h t(\gamma)-h t(\beta)=1$, then $E_{\gamma}=w \llbracket E_{\beta}, E_{\gamma-\beta} \rrbracket$ for some $w \in \mathbb{R}^{\times}$. In this case, since $c_{\gamma}=1$, similarly to the proof in (1), we have:

$$
\left\langle E_{\gamma-\beta} \otimes E_{\beta}, \Delta^{\prime}\left(E_{\gamma}\right)\right\rangle=-w^{-1}(-1)^{p(\gamma-b) p(\beta)} q^{(\gamma-\beta, \beta)}\left\langle E_{\gamma}, E_{\gamma}\right\rangle
$$

By (10.1.3), we have:

$$
\begin{gathered}
\left\langle E_{\alpha}, E_{\alpha}\right\rangle=z\left\langle E_{\beta} \otimes E_{\gamma}-(-1)^{p(\beta) p(\gamma)} q^{-(\beta, \gamma)} E_{\gamma} \otimes E_{\beta}, \Delta^{\prime}\left(E_{\alpha}\right)\right\rangle \\
=z^{2}\left\langle E_{\beta} \otimes E_{\gamma}-(-1)^{p(\beta) p(\gamma)} q^{-(\beta, \gamma)} E_{\gamma} \otimes E_{\beta},\right. \\
\mathbb{\{}\left\{E_{\beta} \otimes 1+L_{\beta} \otimes E_{\beta}\right\},\left\{E_{\gamma} \otimes 1\right.
\end{gathered}
$$




$$
\begin{aligned}
& -\delta_{h t(\gamma), h t(\beta)+1} w^{-1}(-1)^{p(\beta) p(\gamma-\beta)} q^{(\beta, \gamma-\beta)}\left\langle E_{\gamma}, E_{\gamma}\right\rangle\left\langle E_{\beta}, E_{\beta}\right\rangle^{-1} \\
& \left.\left.\cdot E_{\gamma-\beta} L_{\gamma-\beta} \otimes E_{\beta}+L_{\gamma} \otimes E_{\gamma}\right\} \rrbracket\right\rangle \\
= & -z^{2}(-1)^{p(\beta) p(\gamma)} q^{-(\beta, \gamma)}\left\{(-1)^{p(\beta) p(\gamma)}\left(q^{(\beta, \gamma)}-q^{-(\beta, \gamma)}\right)\right. \\
& \left.-\delta_{h t(\gamma), h t(\beta)+1} w^{-2}(-1)^{p(\beta) p(\gamma-\beta)} q^{(\beta, \gamma-\beta)}\left\langle E_{\gamma}, E_{\gamma}\right\rangle\left\langle E_{\beta}, E_{\beta}\right\rangle^{-1}\right\} \\
& \left\langle E_{\gamma} \otimes E_{\beta}, E_{\gamma} L_{\gamma} \otimes E_{\beta}\right\rangle \\
= & -z^{2}\left\{\left(1-q^{-2(\beta, \gamma)}\left\langle E_{\gamma}, E_{\gamma}\right\rangle\left\langle E_{\beta}, E_{\beta}\right\rangle\right.\right. \\
& \left.-\delta_{h t(\gamma), h t(\beta)+1} w^{-2}(-1)^{p(\beta)} q^{(-\beta, \beta)}\left\langle E_{\gamma}, E_{\gamma}\right\rangle^{2}\right\} .
\end{aligned}
$$

Since $c_{\gamma}=c_{\beta}=1$, using results in case (1), we can get $\left\langle E_{\alpha}, E_{\alpha}\right\rangle$.

10.4 .

Proposition 10.4.1. (The Poincaré-Birkhoff-Witt theorem for $\mathscr{N}_{+}$and $\left.N_{+}\right)$

(i) The R-module $\mathscr{N}_{+}$is a free module with a basis

$$
\left\{\prod_{\alpha \in \Phi_{+}^{\text {red }}}^{<} E_{\alpha}^{n_{\alpha}}\left(n_{\alpha} \in Z_{+} \text {if }(\alpha, \alpha) \neq 0, n_{\alpha}=0,1 \text { if }(\alpha, \alpha)=0\right)\right\}
$$

(ii) Let $N_{+}$and $I_{+}$(resp. $\mathscr{N}_{+}$and $\mathscr{I}_{+}$) be the $R$-algebra and the ideal defined in 2.9 (resp. 4.2) respectively. Then $N_{+}=\mathscr{N}_{+}$and $I_{+}=\mathscr{I}_{+}$.

Proof. By Lemma 10.3.1, $\left\langle E_{\alpha}, E_{\alpha}\right\rangle \neq 0$. Therefore, from Proposition 5.3.1 and Lemma 10.2.1, the proposition follows.

10.5. Let $\left(\mathscr{E}, \Pi=\left\{\alpha_{1}, \cdots, \alpha_{n}\right\}, p\right)$ and $D$ be the triple system and the diagonal matrix described in 3.1. Let $U_{h}=U_{h}(\Pi, p)=U_{h}((\mathscr{E}, \Pi, p) D)$ be the $R$-Hopf superalgebra defined in Corollary 2.9.11 for the $R$-Hopf algebra $U_{h}^{\sigma}=U_{h}^{\sigma}((\mathscr{E}, \Pi, p), D)$. Note that $U_{h}$ is a topologically free $R$-module. Denote the submodule of $U_{h}$ of elements of even (resp. odd) parity by $U_{h, 0}$ (resp. $\left.U_{h, 1}\right)$. Then $U_{h}=U_{h, 0} \oplus U_{h, 1}$. Put $[X, Y]=X Y-(-1)^{i j} \quad Y X$ for $X \in U_{h, i}$ 
and $Y \in U_{h, j}$. Let $F_{\alpha}=E_{\alpha}^{\circ} \sigma^{p(\alpha)} \in U_{h}^{\sigma}\left(\alpha \in \Phi_{+}^{\text {red }}\right)$. As an immediately consequence of Proposition 10.4.1, we have:

Theorem 10.5.1. (i) The R-module $U_{h}=U_{h}(\Pi, p)=U_{h}((\mathscr{E}, \Pi, p), D)$ has a topological basis

$$
\prod_{\alpha \in \Phi_{+}^{\text {red }}}^{<} E_{\alpha}^{m_{\alpha}} \cdot \prod_{1 \leq i \leq N} H_{\bar{\varepsilon}_{i}}^{l_{i}} \cdot \prod_{\alpha \in \Phi_{+}^{\text {red }}}^{<} F_{\alpha}^{n_{\alpha}}
$$

$\left(l_{i} \in \mathbb{Z}, m_{\alpha}, n_{\alpha} \in Z_{+}\right.$if $(\alpha, \alpha) \neq 0, m_{\alpha}, n_{\alpha}=0,1$ if $\left.(\alpha, \alpha)=0\right)$.

(ii) As an $R$-superalgebra, $U_{h}$ is topologically defined by the generators, $E_{i}, F_{i}(1 \leq i \leq n), H \in \mathscr{H}$ with the parities $p\left(E_{i}\right)=p\left(F_{i}\right)=p\left(\alpha_{i}\right), p(H)=0$ and the relations

$$
\left[H_{1}, H_{2}\right]\left(H_{1}, H_{2} \in \mathscr{H}\right)
$$

(10.5.4) $\left[H, E_{i}\right]=\alpha_{i}(H) E_{i},\left[H, F_{i}\right]=-\alpha_{i}(H) F_{i}$,

$$
\left[E_{i}, F_{j}\right]=\delta_{i j} \frac{\operatorname{sh}\left(h H_{\alpha_{1}}\right)}{\operatorname{sh}\left(h d_{i}\right)},
$$

(10.5.6) The relations of $E_{i}$ 's defined in Definition 4.2.1.

(10.5.7) The relations (10.5.6) with $E_{i}$ 's replaced for $F_{i}$ 's.

(iii) The Hopf superalgebra structure of $U_{h}$ is given by the coproduct $\dot{\Delta}$, the antipode $\dot{S}$ and the counit $\dot{\varepsilon}$ such that $\left(\right.$ Here put $K_{i}=\exp \left(h H_{\alpha_{i}}\right)$.)

$$
\begin{aligned}
& \dot{\Delta}(H)=H \otimes 1+1 \otimes H(H \in \mathscr{H}), \\
& \dot{\Delta}\left(E_{i}\right)=E_{i} \otimes 1+K_{i} \otimes E_{i}, \dot{\Delta}\left(F_{i}\right)=F_{i} \otimes K_{i}^{-1}+1 \otimes F_{i}, \\
& \dot{S}(H)=-H, \dot{S}\left(E_{i}\right)=-K_{i}^{-1} E_{i}, \dot{S}\left(F_{i}\right)=-F_{i} K_{i}, \\
& \dot{\varepsilon}(H)=\dot{\varepsilon}\left(E_{i}\right)=\dot{\varepsilon}\left(F_{i}\right)=0 .
\end{aligned}
$$


Theorem 10.5.8. Let $\hat{U}_{h}=\hat{U}_{h}((\mathscr{E}, \Pi, p), D)$ be an R-Hopf superalgebra defined by $\hat{U}_{h}=U_{h} / \hat{z} \cdot U_{h}$ where $\hat{z}=\left\{H \in \mathscr{H} \mid \alpha_{i}(H)=0(1 \leq i \leq n)\right\}$. Then $\hat{U}_{h}$ is topologically free. Let $\mathscr{G}$ be the complex simple Lie superalgebra defined for $(\mathscr{E}, \Pi, p)$. Let $U(\mathscr{G})$ be the enveloping superalgebra of $\mathscr{G}$. Then $U(\mathscr{G})=\hat{U}_{h}(\mathscr{G}) /$ $h \hat{U}_{h}(\mathscr{G})$ as a $C$-Hopf superalgebra.

Proof. The topological freeness of $\hat{U}_{h}(\mathscr{G})$ is clear. By Proposition 10.5.1, we see that there exists a natural $C$-Hopf superalgebra homomorphism $\omega: \hat{U}_{h}(\mathscr{G}) / h \hat{U}_{h}(\mathscr{G}) \rightarrow U(\mathscr{G})$ such that $\omega\left(E_{i}\right), \omega\left(F_{i}\right)$ and $\omega(\hat{H})(\hat{H} \in \mathscr{H} / \hat{z})$ are Serre generators of $U(\mathscr{G})$. From the Poincaré-Birkhoff-Witt theorem for Lie superalgebras (see [3]), it follows that a P.B.W.-type basis of $\hat{U}_{h}(\mathscr{G}) / h \hat{U}_{h}(\mathscr{G})$ arising from (10.5.2) is sent to a basis of $U(\mathscr{G})$. Hence $\omega$ is isomorphism.

As an immediately consequence of Proposition 10.5.1 (ii) and Theorm 10.5.8, we have:

Corollary 10.5.9. By substituting 0 for $h$ in (10.5.3-7), we get defining relations of $U(\mathscr{G})$.

10.6. Here we give the main theorem. Let $\varepsilon_{i}$ 's be basis elements of $\mathscr{E}$ such that $\left(\varepsilon_{i}, \varepsilon_{j}\right)=\delta_{i j}$. Put $t_{0}=\sum_{i=1}^{n} H_{\varepsilon_{i}} \otimes H_{\varepsilon_{i}} \in \mathscr{H} \otimes \mathscr{H}$. Let $e(u ; t)=$ $\sum_{n=0}^{\infty}\left(u^{n} / \Psi_{n}(t)\right)$ be the formal power series called the " $q$-exponential". Put $u(\alpha)=(-1)^{h t(\alpha)} b(\alpha)^{-1}$.

Theorem 10.6.1. (Universal R-matrix of $U_{h}^{\sigma}$ ) Let $\mathscr{R}$ be an element of $U_{h}^{\sigma} \hat{\otimes} U_{h}^{\sigma}$ defined by

$$
\begin{aligned}
\mathscr{R}= & \left\{\prod_{\alpha \in \Phi_{+}^{\text {red }}}^{<} e\left(\left(q^{d_{\alpha}}-q^{-d_{\alpha}}\right) u(\alpha) E_{\alpha} \otimes F_{\alpha} \sigma^{p(\alpha)} ;(-1)^{p(\alpha)} q^{(\alpha, \alpha)}\right)\right\} \\
& \cdot\left\{\frac{1}{2} \sum_{c, d \in\{0,1\}}(-1)^{c d} \sigma^{c} \otimes \sigma^{d}\right\} \cdot \exp \left(-h t_{0}\right) .
\end{aligned}
$$

Then $\left(U_{h}^{\sigma}, \Delta, \mathscr{R}\right)$ is a quasi-triangular Hopf algebra.

Proof. Use Lemma 2.9.10, Lemma 10.2.1, Lemma 10.3 .1 and 
Proposition 10.4.1. Here we note the facts $\Omega^{\prime}\left(E_{\alpha}\right)=E_{\alpha}, \Omega^{\prime}\left(F_{\alpha}\right)=$ $\left.\prod_{i=1}^{n}\left(q^{-d_{i}}-q^{d_{i}}\right)^{c_{i}}\right\} F_{\alpha}$ for $\alpha=c_{1} \alpha_{1}+\cdots+c_{n} \alpha_{n} \in \Phi_{+}^{\mathrm{red}}$.

10.7. Here we give the $R$-matrix $\rho \otimes \rho(\mathscr{R})$ for the fundamental representation $\rho$ of $U_{h}^{\sigma}$ of type $A_{N-1}$.

The fundamental representation $\rho U_{h}^{\sigma} \rightarrow M_{N}(C)$ is defined by $\rho\left(E_{i}\right)=e_{i, i+1}$, $\rho\left(F_{i}\right)=\bar{d}_{i} e_{i+1, i}(1 \leq i \leq N-1), \rho\left(H_{\bar{\varepsilon}_{i}}=\bar{d}_{i} e_{i i}(1 \leq i \leq N)\right.$ and $\rho(\sigma)=\sum_{i=1}^{N} \bar{d}_{i} e_{i i}$. Put $q=e^{h}$. In this case, we have:

$$
\rho \otimes \rho(\mathscr{R})=\sum_{i<j}\left(q^{-1}-q\right) e_{i j} \otimes e_{j i}+\sum_{i, j} q^{-\bar{d}_{i} \delta_{i j}}(-1)^{\frac{1}{4}\left(1-\bar{d}_{i}\right)\left(1-\bar{d}_{j}\right)} e_{i i} \otimes e_{j j}
$$

Moreover $\mathscr{R}(x)=x(\rho \otimes \rho(\mathscr{R}))-x^{-1}(\rho \otimes \rho(\mathscr{R}))^{-1}$ satisfies the YangBaxter equation with a spectral parameter:

$$
\mathscr{R}(x)_{12} \mathscr{R}(x y)_{13} \mathscr{R}(y)_{23}=\mathscr{R}(y)_{23} \mathscr{R}(x y)_{13} \mathscr{R}(x)_{12}
$$

This $R$-matrix was discovered by Perk and Schultz [15] (see also [14]).

\section{§1. Remark on the Necessity of the Defining Relations}

11.1. It can be shown that none of the relations (i)-(vi) in Definition 4.2.1 can be dropped. Below we only show it for the relation (v). The other relations can be treated quite similarly.

11.2. We use the notation in 2.1. Let $\boldsymbol{I}_{+}$be an ideal of $\tilde{N}_{+}$generated by the elements of Definition 4.2.1 (i)-(iv). Put $N_{+}=\tilde{N}_{+} / \mathbb{I}_{+}$. We define an ideal $\mathbb{I}_{-}$of $\tilde{N}_{-}$in a similar way. Let $\mathbb{L}$ be the ideal of $\tilde{U}_{h}^{\sigma} h$-adically generated by the elements in $\mathbb{I}_{+} \cup \mathbb{I}_{-}$. Put $U_{h}^{\sigma}=\tilde{U}_{h}^{\sigma} / \mathbb{L}$.

Lemma 11.2.1. Let $i_{+}: \mathbb{N}_{+} \rightarrow \mathbb{U}_{h}^{\sigma}$ be an $R$-algebra map defined by $\mathrm{x}+\mathbb{I}_{+} \rightarrow x+\mathbb{L}$. Then $i_{+}$is injective. 
Proof. By direct computations, we see that $F_{i} \cdot I_{+} \subset I_{+} \cdot F_{i}+I_{+}(1 \leq i \leq n)$. Hence $\boldsymbol{L}_{+}=\overline{\boldsymbol{I}_{+} \subseteq\left[\mathscr{H}^{R}\right] R\langle\sigma\rangle \tilde{N}_{-}}$is an ideal of $\tilde{U}_{h}^{\sigma}$. Similarly, we see that $\boldsymbol{L}_{-}=\tilde{\bar{N}}_{+} \subseteq\left[\mathscr{H}^{R}\right] R\langle\sigma\rangle \boldsymbol{I}_{-}$is also an ideal of $\tilde{U}_{h}^{\sigma}$. Hence $\boldsymbol{L}=\boldsymbol{L}_{+}+\boldsymbol{L}_{-}$. In particular, we see that $\boldsymbol{L} \cap \tilde{N}_{+}=I_{+}$. This completes the proof.

11.3. For $v \in P_{+}$, let $\boldsymbol{N}_{+, v}=\tilde{N}_{+, v}+I_{+} \subset N_{+}$and $\boldsymbol{I}_{+, v}=\tilde{N}_{+, v} \cap I_{+}$. Then $\boldsymbol{N}_{+, v}=\tilde{N}_{+, v} / \boldsymbol{I}_{+, v}$.

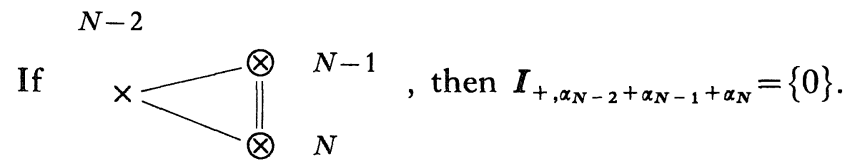

In particular, rank $N_{+, \alpha_{i-1}+2 \alpha_{i}+\alpha_{i+1}}=6$. Hence Poincaré-Birkhoff-Witt type theorem can not hold for $U_{h}^{\sigma}$.

11.4. Let $U_{h}^{\dagger}$ be the Hopf superalgebra called the "quantized Kac-Moody superalgebra" in [9]. Here we understand that $\boldsymbol{U}_{h}^{\dagger}$ is defined as an $h$-adic $R$-Hopf superalgebra. Even if we take the Note added in proof in [9] into account, we can show that there exists a natural epimorphism $\left(\boldsymbol{U}_{h}^{\dagger}\right)^{\sigma} \rightarrow \boldsymbol{U}_{h}^{\sigma}$ of Hopf algebras. Hence, for $\boldsymbol{U}_{h}^{\dagger}$, a P.B.W. type theorem can not hold contrary to their assertion (Proposition 3.3 and Remark under it) in [9].

\section{Acknowledgements}

I would like to express my heartfelt thanks to Professor M. Noumi, Professor T. Tanisaki, Professor J. Murakami and Professor M. Okado for valuable communication. Specially I thank Professor T. Tanisaki for showing me a proof of the result on the "quantum double construction". I also thank Professor M. Rosso for sending me his preprint of [16] and Professor E. Date for informing me about the existence of the preprint [9] of Khoroshkin and Tolstoy.

\section{References}

[1] Abe., E., Hopf algebras, Cambridge Univ. Press, Cambridge-New York, 1980. 
[ 2 ] Baxter, R.J., Exactly solved models in statistical mechanics, Academic Press, London, 1982.

[ 3 ] Corwin, L., Ne'eman, Y. and Sternberg, S., Graded Lie algebras in mathematics and physics, Rev. Mod. Phys., 47 (1975), 573-604.

[4] Drinfeld, V.G., Quantum groups, Proc. Int. Congr. Math., Berkeley, 1 (1986); Amer. Math. Soc. (1988), 798-820.

[ 5 ] Jimbo, M., A $q$-difference analogue of $U(\mathscr{G})$ and the Yang-Baxter equation, Lett. Math. Phys., 10 (1985), 63-69.

[ 6 ] Kac, V.G., Infinite dimensional Lie algebras, 3rd ed., Cambridge Univ. Press, Cambridge, 1990.

[ 7 ] Kac, V.G., Lie superalgebras, Adv. Math., 26 (1977), 8-96.

[8] Kirillov, A.N. and Reshetikhin, N.Yu., $q$-Weyl group and a multiplicative formula for universal R-matrix, Comm. Math. Phys., 134 (1990), 421-431.

[ 9 ] Khoroshkin, S.M. and Tolstoy, V.N., Universal $R$-matrix for quantized (super)algebras, Comm. Math. Phys, 141 (1991), 599-617.

[10] Levendorskii., S. and Soibelman, Ya., Some applications of quantum Weyl group, J. Geom. Phys., 7 (1990), 241-254.

[11] Lusztig, G., Finite dimensional Hopf algebras arising from quantized universal enveloping algebras, J. Amer. Math. Soc., 3 (1990), 257-296.

[12] — Quantum groups at roots of 1, Geom. Dedicata, 35 (1990), 89-144.

[13] Matsumura, H., Commutative Algebra, Second ed. London: Benjamin, 1980.

[14] Okado, M. and Yamane, H., $R$-matrices with gauge parameters and multi-parameter quantized enveloping algebras, Special Functions (Proceedings of Hayashibara Forum 1990 held in Fujisaki Institute, Okayama, Japan, August 16-20, 1990) ed. by M. Kashiwara and T. Miwa, Springer-Verlag, Tokyo, New York, Berlin, 1991, 289-293.

[15] Perk, J.H.H. and Schultz, C.L., Families of commuting transfer matrices in $q$-state vertex models, Non-linear Integrable system-Classical theory and quantum theory (Proceedings of RIMS symposium, Kyoto, Japan, 13-16 May 1981) ed. by M. Jimbo and T. Miwa, World Scientific, Singapore, 1983, 326-343.

[16] Rosso, M., An analogue of P.B.W. Theorem and the Universal $R$-matrix for $U_{h} s l(N+1)$, Comm. Math. Phys., 124 (1989), 307-318.

[17] Scheunert, M., The theory of Lie superalgebras, Lecture Note in Math. 716, Springer, Berlin, 1979.

[18] Takeuchi, M., Finite-dimensional representations of the quantum Lorents group, Comm. Math. Phys., 144 (1992), 557-580.

[19] Tanisaki, T., Harish-Chandra isomorphism for quantum algebras, Comm. Math. Phys., 127 (1990), 555-571.

[20] - Killing forms, Harish-Chandra isomorphisms, and universal $R$-matrices for quantum algebras, Infinite Analysis (Proceedings of RIMS symposium, Kyoto, Japan, June-August 1991) ed. by A. Tsuchiya, T. Eguchi and M. Jimbo, World scientific, Singapore, 1992, 941-961.

[21] Turaev, T.G., The Yang-Baxter equation and invariants of links, Invent. Math., 92 (1988), 527-553.

[22] Yamane, H., Universal $R$-matrices for quantum groups associated to simple Lie superalgebras, Proc. Japan Acad., Ser. A, No.4 (1991), 108-112.

Note added in proof: In this paper, for the datum $((E, \Pi, p), D)$, we first defined the Hopf superalgebra $U_{h}=U_{h}(\Pi, p)=U_{h}((\mathscr{E}, \Pi, p), D)$ in an abstract manner in $\S 2$. Later, in Theorem 2.10.1, we showed that, if $((\mathscr{E}, \Pi, p), D)$ corresponds to a symmetrizable Kac-Moody Lie algebra $G$, then our $U_{h}$ coincides with the Drinfeld-type quantized enveloping algebra $U_{h}(G)$ topologically defined over $C[[h]]$. Namely, in this case, the defining relations satisfied by the Chevalley generators of $U_{h}$ are the $q$-Serre relations. After this paper has been submitted, the author learned that the same definition and result for the Jimbo-type quantized enveloping algebra 
$U_{q}(\boldsymbol{G})$ defined over $\boldsymbol{C}(q)$ are given in the recent book of Prc. ${ }^{c}$. F Lusztig (Introduction to Quantum Groups, Birkhäuser, Boston, 1993). 
